\title{
A new energy-momentum time integration scheme for non-linear thermo-mechanics
}

\author{
R. Ortigosa ${ }^{\dagger 1}$, A. J. Gil ${ }^{\ddagger 3}$, J. Martínez-Frutos ${ }^{\dagger 2}$, M. Franke ${ }^{\diamond}$, J. Bonet ${ }^{\star}$ \\ $\dagger$ Computational Mechanics and Scientific Computing Group, \\ Technical University of Cartagena, Campus Muralla del Mar, 30202, Cartagena (Murcia), Spain \\ $\ddagger Z i e n k i e w i c z$ Centre for Computational Engineering, College of Engineering \\ Swansea University, Bay Campus, SA1 8EN, United Kingdom \\ $\diamond$ Institute of Mechanics, Karlsruhe Institute of Technology, 76131 Karlsruhe, Germany \\ * University of Greenwich, London, SE10 9LS, United Kingdom
}

\begin{abstract}
The aim of this paper is the design a new one-step implicit and thermodynamically consistent Energy-Momentum (EM) preserving time integration scheme for the simulation of thermo-elastic processes undergoing large deformations and temperature fields. Following [12], we consider well-posed constitutive models for the entire range of deformations and temperature. In that regard, the consideration of polyconvexity inspired constitutive models and a new tensor cross product algebra are shown to be crucial in order to derive the so-called discrete derivatives, fundamental for the construction of the algorithmic derived variables, namely the second PiolaKirchoff stress tensor and the entropy (or the absolute temperature). The proposed scheme inherits the advantages of the EM scheme recently published by Franke et al. [17], whilst resulting in a simpler scheme from the implementation standpoint. A series of numerical examples will be presented in order to demonstrate the robustness and applicability of the new EM scheme. Although the examples presented will make use of a temperature-based version of the EM scheme (using the Helmholtz free energy as the thermodynamical potential and the temperature as the thermodynamical state variable), we also include in an Appendix an entropy-based analogue EM scheme (using the internal energy as the thermodynamical potential and the entropy as the thermodynamical state variable).
\end{abstract}

Keywords: finite element method, nonlinear thermo-elastodynamics, energy-momentum scheme, structure-perserving discretisation.

\section{Introduction}

The development of thermo-elastic constitutive models for the simulation of materials undergoing external mechanical and thermal loading has been the focus of intensive study in numerous References $[17,25]$. These constitutive models are typically based on an invariant representation of the Helmholtz free energy functional, defined in terms of the deformation gradient tensor $\boldsymbol{F}$ (through the objective right Cauchy-Green tensor $\boldsymbol{C}$ ) and the absolute temperature $\theta$ (temperature in the sequel). Based on this energy (potential) functional, many authors [17, 25] have proposed temperature-based consistent implicit energy momentum (EM) time integration schemes for the long term simulation of structural components governed by this Helmholtz potential. This type of time integration schemes are well-known for their robustness and stability properties due to their

\footnotetext{
${ }^{1}$ Corresponding author: r.ortigosa@swansea.ac.uk

${ }^{2}$ Corresponding author: marlon.franke@kit.edu@kit

${ }^{3}$ Corresponding author: a.j.gil@swansea.ac.uk
} 
structure-preserving features, making them ideal for long term stable simulations. We purposely use the term temperature-based to stress the fact that this type of algorithms rely on the temperature field as the thermodynamical state variable and, as such, this temperature represents one of the unknowns to be solved.

EM schemes are regarded as both elegant and robust because they are endowed by construction with the discrete analogue of the conservation properties of the continuum, namely the conservation of total energy, linear and angular momenta. The consistency of EM schemes refers to their ability to preserve (or dissipate for non-reversible constitutive models) the total energy of a system in agreement with the laws of thermodynamics [20, 7, 17, 25]. Consistency of these methods is attained by replacing the (exact) partial derivatives of the Helmholtz free energy functional with respect to its arguments $(\boldsymbol{C}$ and $\theta)$ with their carefully designed algorithmic counterparts. These algorithmic partial derivatives, also known as discrete derivatives [17, 7, 27, 18, 31], are formulated in compliance with the so-called directionality property [18]. Unfortunately, the success of current temperature-based EM consistent integrators rely on the introduction of discrete derivatives incorporating consistency restoring terms [21] which are not that straightforward to be systematised and generalised, especially if interested in pursuing multi-physics applications beyond thermo-mechanics.

An alternative approach uses the entropy-based GENERIC framework, where the entropy $\eta$ is considered as the thermodynamical state variable (see the works of Romero [29] and Conde [25]), where EM schemes have been also successfully developed. However, a well-accepted difficulty of entropy-based formulations is the non-trivial consideration of temperature boundary conditions. Indeed, unless relatively simple thermo-mechanical models are considered, a (computationally expensive) Newton-Raphson type procedure is required in order to solve a nonlinear equation relating $\eta$ and $\theta$ on the part of the boundary subjected to prescribed temperature (at each boundary quadrature point). This is one of the main reasons to prefer the Helmholtz free energy functional as a thermodynamic potential over the internal energy and, thus, the temperature over the entropy as the thermodynamical state variable.

Very recently, Franke et. al. [17] have proposed a novel EM scheme in the context of thermoelasticity, by taking advantage of the concept of isothermal polyconvexity $[1,2,3,4,30]$ and the use of a novel tensor cross product pioneered by de Boer [15] and re-discovered in the context of nonlinear continuum mechanics by Bonet et al. [10, 9, 8]. In essence, the authors propose the consideration of four discrete derivatives which are used to form algorithmic versions of the second Piola-Kirchoff stress tensor and the entropy of the system. In addition to the discrete derivative with respect to the temperature, three further discrete derivatives are presented, which represent the algorithmic counterparts of the work conjugates of the right Cauchy-Green deformation tensor, its co-factor and its determinant. This strategy leads to simplified expressions of the algorithmic second Piola-Kirchoff stress tensor and entropy, when comparing against those obtained following the classical approach [18]. Finally, as the EM scheme in [17] relies on the re-definition of the internal energy of the system in terms of the temperature, this ultimately entails a certain level of complexity in the derivation of the discrete derivatives (refer to Appendix B).

The aim of the current paper is the development of a new polyconvexity inspired temperaturebased EM scheme which uses the Helmholtz free energy functional as the fundamental thermodynamical potential. As a result, the new EM scheme will be shown to inherit the advantages of that of [17] (i.e. consistency, stability, conservation) whilst, in addition, resulting in dramatically far simpler algorithmic expressions. We conceive the simplification brought forward by the new EM scheme as a crucial preliminary step in order to bridge the gap with recently published EM schemes developed by the authors in the context of electro-elasticity [27] and, therefore, seek extension to thermo-electro-elasticity.

The outline of this paper is as follows: in Section 2, some basic principles of kinematics are presented. The governing equations in nonlinear thermo-elastodynamics are also presented in 
this section. Section 3 introduces the Helmholtz free energy functional and the internal energy functional. Section 4 presents the weak forms associated with the governing equations in thermoelastodynamics. These will help introducing the new temperature-based one-step implicit EM time integrator scheme for thermo-elastodynamics in Section 5. Section 6 briefly describes the finite element implementation of the new time integrator scheme and Section 7 presents a series of numerical examples in order to validate the conservation properties and robustness of the new scheme. Finally, Section 8 provides some concluding remarks. Appendix A presents an entropybased EM scheme, counterpart of the temperature-based scheme pursued in this paper. Appendix $\mathrm{B}$ outlines the definition of the discrete derivative expressions featuring in the proposed time integrator in Section 5. Appendix C summarises the EM scheme in Reference [17], illustrating the differences between this and the new EM proposed. Appendix D presents the steps that need to be carried out in order to derive the thermo-elastic constitutive model presented in Section 3.1.1.

\section{Nonlinear continuum thermo-mechanics}

A brief introduction into nonlinear continuum mechanics and the relevant governing equations will be presented in this section.

\subsection{Kinematics: motion and deformation}

Let us consider the motion of a continuum with reference configuration $\mathcal{B}_{0} \in \mathbb{R}^{3}$ of boundary $\partial \mathcal{B}_{0}$ and unit outward normal $\boldsymbol{N}$ (refer to Figure 1). After the motion, the continuum occupies a deformed configuration $\mathcal{B} \in \mathbb{R}^{3}$ with boundary $\partial \mathcal{B}$ and unit outward normal $\boldsymbol{n}$. The deformation mapping $\boldsymbol{\phi}(\boldsymbol{X}, t)$ links a material particle from the reference configuration $\boldsymbol{X} \in \mathcal{B}_{0}$ to the deformed configuration $\boldsymbol{x} \in \mathcal{B}$ according to $\boldsymbol{x}=\boldsymbol{\phi}(\boldsymbol{X}, t)$. Associated with the mapping $\boldsymbol{\phi}(\boldsymbol{X}, t)$ it is possible to define the two-field deformation gradient tensor $\boldsymbol{F}[11,19,16,5]$ as

$$
\boldsymbol{F}=\nabla_{0} \boldsymbol{\phi}(\boldsymbol{X}, t) ; \quad F_{i I}=\frac{\partial \phi_{i}}{\partial X_{I}}
$$

Associated with $\boldsymbol{F}$ it is possible to define its co-factor $\boldsymbol{H}$ and its Jacobian $J$, defined as

$$
\boldsymbol{H}=(\operatorname{det} \boldsymbol{F}) \boldsymbol{F}^{-T} ; \quad J=\operatorname{det} \boldsymbol{F} .
$$

Equivalent expressions to those in (2) for both $\boldsymbol{H}$ and $J$ can be obtained by making use of the tensor cross product operation, introduced by de Boer [15] and rediscovered by Bonet et al. $[10]$ in the context of nonlinear solid mechanics, as $^{4}$

$$
\begin{aligned}
\boldsymbol{H} & =\frac{1}{2} \boldsymbol{F} \times \boldsymbol{F} ; & H_{i I} & =\frac{1}{2} \mathcal{E}_{i j k} \mathcal{E}_{I J K} F_{j J} F_{k K} ; \\
J & =\frac{1}{3} \boldsymbol{H}: \boldsymbol{F} ; & J & =\frac{1}{3} H_{i I} F_{i I} .
\end{aligned}
$$

\footnotetext{
${ }^{4} \mathcal{E}_{i j k}\left(\right.$ or $\left.\mathcal{E}_{I J K}\right)$ symbolises the third order alternating tensor components and the use of repeated indices implies summation, unless otherwise stated. Lower case indices $\{i, j, k\}$ will be used to represent the spatial configuration whereas capital case indices $\{I, J, K\}$ will be used to represent the material configuration.
} 
2.2. Governing equations in nonlinear thermo-mechanics: conservation of linear momentum and angular momentum

The local form of the conservation of linear momentum [19] can be written as

$$
\begin{aligned}
\rho_{0} \dot{\boldsymbol{v}}-\operatorname{DIV}(\boldsymbol{F} \boldsymbol{S})-\boldsymbol{f}_{0} & =\mathbf{0} ; & & \text { in } \mathcal{B}_{0} ; \\
(\boldsymbol{F} \boldsymbol{S}) \boldsymbol{N} & =\boldsymbol{t}_{0} ; & & \text { on } \partial_{\boldsymbol{t}} \mathcal{B}_{0} ; \\
\boldsymbol{\phi} & =\overline{\boldsymbol{\phi}} ; & & \text { on } \partial_{\phi} \mathcal{B}_{0} ; \\
\left.\dot{\boldsymbol{\phi}}\right|_{t=0} & =\dot{\bar{\phi}} ; & & \text { in } \mathcal{B}_{0} ; \\
\left.\boldsymbol{\phi}\right|_{t=0} & =\overline{\boldsymbol{\phi}} ; & & \text { in } \mathcal{B}_{0},
\end{aligned}
$$

where $\rho_{0}: \mathcal{B}_{0} \rightarrow \mathbb{R}^{+}$represents the mass density of the continuum in the reference configuration, $\boldsymbol{v}$ the velocity field and $(\dot{\bullet}):=\frac{d(\bullet)}{d t}$ denotes differentiation with respect to time. Furthermore, $\boldsymbol{f}_{0}$ represents a body force per unit undeformed volume $\mathcal{B}_{0}$ and $\boldsymbol{t}_{0}$, the traction force per unit undeformed area applied on $\partial_{\boldsymbol{t}} \mathcal{B}_{0} \subset \partial \mathcal{B}_{0}$, such that $\partial_{\boldsymbol{t}} \mathcal{B}_{0} \cup \partial_{\phi} \mathcal{B}_{0}=\partial \mathcal{B}_{0}$ and $\partial_{t} \mathcal{B}_{0} \cap \partial_{\phi} \mathcal{B}_{0}=\emptyset$. Finally, $\boldsymbol{S}$ represents the second Piola-Kirchhoff stress tensor and the local conservation of angular momentum leads to the well-known tensor condition $\boldsymbol{S}=\boldsymbol{S}^{T}$.

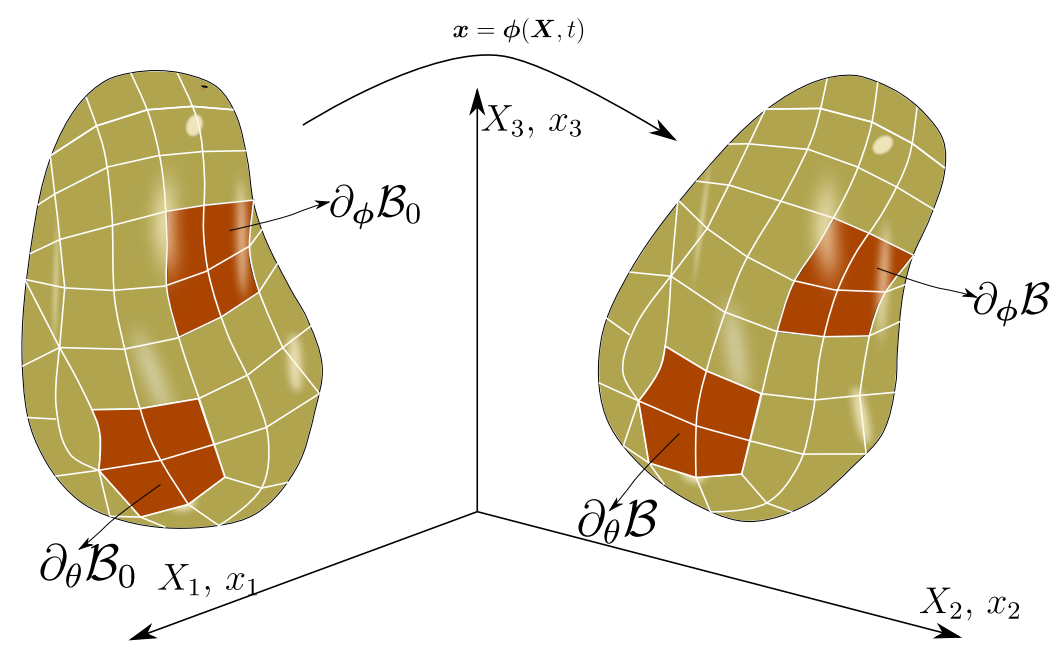

Figure 1: The mapping $\phi$ and the mechanical and thermal portions of the boundary where Dirichlet conditions are imposed in the reference (and deformed) configurations, namely $\partial_{\phi} \mathcal{B}_{0}\left(\right.$ and $\left.\partial_{\phi} \mathcal{B}\right)$ and $\partial_{\theta} \mathcal{B}_{0}\left(\right.$ and $\partial_{\theta} \mathcal{B}$ ).

\subsection{Governing equations in non-linear thermo-mechanics: conservation of energy}

In the absence of internal state variables (i.e. plastic strain), the local form of the balance of energy can be written in a Lagrangian setting as

$$
\begin{aligned}
\theta \dot{\eta}+\mathrm{DIV} \boldsymbol{Q}-R_{\theta} & =0 ; & & \text { in } \mathcal{B}_{0} ; \\
\boldsymbol{Q} \cdot \boldsymbol{N} & =-Q_{\theta} ; & & \text { on } \partial_{Q} \mathcal{B}_{0} ; \\
\theta & =\bar{\theta} ; & & \text { on } \partial_{\theta} \mathcal{B}_{0} ; \\
\left.\theta\right|_{t=0} & =\bar{\theta} ; & & \text { in } \mathcal{B}_{0},
\end{aligned}
$$

where $\theta$ is the absolute temperature field and $\eta$ and $\boldsymbol{Q}$, the entropy and heat flux per unit undeformed volume $\mathcal{B}_{0}$, respectively. In addition, $R_{\theta}$ represents the heat source per unit undeformed volume $\mathcal{B}_{0}$ and $Q_{\theta}$, the heat source per unit undeformed area applied on $\partial_{Q} \mathcal{B}_{0} \subset \partial \mathcal{B}_{0}$. In (5), $\partial_{\theta} \mathcal{B}_{0}$ represents the part of the boundary $\partial \mathcal{B}_{0}$ where essential temperature boundary conditions are applied such that $\partial_{Q} \mathcal{B}_{0} \cup \partial_{\theta} \mathcal{B}_{0}=\partial \mathcal{B}_{0}$ and $\partial_{Q} \mathcal{B}_{0} \cap \partial_{\theta} \mathcal{B}_{0}=\emptyset$. In order to develop a suitable EM 
time integrator in the context of thermo-elasticity, we advocate in this work for an alternative but equivalent (at the continuum level) expression of (5), namely

$$
\begin{aligned}
\frac{d}{d t}(\theta \eta)-\dot{\theta} \eta+\mathrm{DIV} \boldsymbol{Q}-R_{\theta} & =0 ; & & \text { in } \mathcal{B}_{0} ; \\
\boldsymbol{Q} \cdot \boldsymbol{N} & =-Q_{\theta} ; & & \text { on } \partial_{Q} \mathcal{B}_{0} ; \\
\theta & =\bar{\theta} ; & & \text { on } \partial_{\theta} \mathcal{B}_{0} ; \\
\left.\theta\right|_{t=0} & =\bar{\theta} ; & & \text { in } \mathcal{B}_{0} .
\end{aligned}
$$

\section{Constitutive equations in nonlinear thermo-elasticity}

The governing equations presented in Section 2 are coupled by means of a suitable constitutive law. The objective of the following section is to introduce some notions on constitutive laws in thermo-elasticity.

\subsection{The Helmholtz free energy}

In the case of thermo-elasticity, the Helmholtz free energy $\Psi$ per unit of undeformed volume can be defined in terms of $\boldsymbol{\nabla}_{0} \boldsymbol{\phi}$ and $\theta$, namely $\Psi=\Psi\left(\boldsymbol{\nabla}_{0} \boldsymbol{\phi}, \theta\right)$ (see [26]), as

$$
\Psi\left(\boldsymbol{\nabla}_{0} \boldsymbol{\phi}, \theta\right)=W(\boldsymbol{F}, \boldsymbol{H}, J, \theta) .
$$

In this work it is assumed that the Helmholtz free energy is rank-one convex with respect to the deformation gradient $\nabla_{0} \phi$ and concave with respect to the absolute temperature $\theta$, i.e.

$$
(\boldsymbol{u} \otimes \boldsymbol{V}): \partial_{\boldsymbol{\nabla}_{0} \phi \boldsymbol{\nabla}_{0} \phi}^{2} \Psi:(\boldsymbol{u} \otimes \boldsymbol{V}) \geq 0, \forall \boldsymbol{u}, \boldsymbol{V} \in \mathbb{R}^{3} ; \quad \partial_{\theta \theta}^{2} \Psi \leq 0 .
$$

Above condition (8) is strongly connected with the notion of material stability [24] and ensures the stability of the system of governing equations (4) and (6) $[13,14]$. A sufficient condition for (8) to hold is obtained when the extended representation $W(\boldsymbol{F}, \boldsymbol{H}, J, \theta)$ is convex with respect to the set $\mathcal{V}=\{\boldsymbol{F}, \boldsymbol{H}, J\}$ (namely, polyconvex with respect to the mechanics $[1,3,4]$ ) and concave with respect to $\theta$, namely

$$
g_{\mathcal{V}}:=\delta \mathcal{V}^{T}:\left[\mathbb{H}_{W}\right]: \delta \mathcal{V} \geq 0, \quad \forall \delta \mathcal{V} ; \quad g_{\theta}:=\partial_{\theta \theta}^{2} W \leq 0,
$$

with $\delta \mathcal{V}$ and the Hessian operator $\left[\mathbb{H}_{W}\right]$ defined as

$$
\delta \mathcal{V}=\left[\begin{array}{c}
\delta \boldsymbol{F} \\
\delta \boldsymbol{H} \\
\delta J
\end{array}\right] ; \quad\left[\mathbb{H}_{W}\right]=\left[\begin{array}{ccc}
\partial_{\boldsymbol{F} F}^{2} W & \partial_{\boldsymbol{F} \boldsymbol{H}}^{2} W & \partial_{\boldsymbol{F} J}^{2} W \\
\partial_{\boldsymbol{H} \boldsymbol{F}}^{2} W & \partial_{\boldsymbol{H}}^{2} W & \partial_{\boldsymbol{H} J}^{2} W \\
\partial_{J \boldsymbol{F}}^{2} W & \partial_{J \boldsymbol{H}}^{2} W & \partial_{J J}^{2} W
\end{array}\right]
$$

For the requirement of objectivity, the energy functional $W(7)$ can be re-expressed in terms of a set of objective arguments as

$$
\Psi\left(\nabla_{0} \phi, \theta\right)=\widetilde{\Psi}(\boldsymbol{C}, \theta)=\widetilde{W}(\boldsymbol{C}, \boldsymbol{G}, C, \theta),
$$

where $\widetilde{\Psi}$ represents the Helmholtz free energy in terms of the right Cauchy-Green strain tensor $\boldsymbol{C}$ and $\theta$ and $\widetilde{W}$ denotes its extended counterpart, expressed in terms of the extended symmetric kinematic set $\{\boldsymbol{C}, \boldsymbol{G}, C\}$, defined as

$$
\boldsymbol{C}=\boldsymbol{F}^{T} \boldsymbol{F} ; \quad \boldsymbol{G}=\frac{1}{2} \boldsymbol{C} \times \boldsymbol{C}=\boldsymbol{H}^{T} \boldsymbol{H} ; \quad C=\frac{1}{3} \boldsymbol{G}: \boldsymbol{C}=J^{2},
$$

and $\theta$. The first law of thermodynamics and the absence of internal state variables leads to

$$
D \widetilde{\Psi}(\boldsymbol{C}, \theta)[\delta \boldsymbol{\phi}]=\boldsymbol{S}: \frac{1}{2} D \boldsymbol{C}[\delta \boldsymbol{\phi}] ; \quad D \widetilde{\Psi}(\boldsymbol{C}, \theta)[\delta \theta]=-\eta \delta \theta
$$


where $D \widetilde{\Psi}[\boldsymbol{a}]$ represents the directional derivative of $\widetilde{\Psi}$ along the field $\boldsymbol{a}$. In addition, the second Piola-Kirchhoff stress tensor $\boldsymbol{S}$ and the material entropy $\eta$ are defined in terms of the derivatives of $\widetilde{\Psi}(\boldsymbol{C}, \theta)$, namely

$$
\boldsymbol{S}=2 \partial_{\boldsymbol{C}} \widetilde{\Psi} ; \quad \eta=-\partial_{\theta} \widetilde{\Psi}
$$

An alternative but equivalent definition of the directional derivatives of $\widetilde{\Psi}(\boldsymbol{C}, \theta)$ to those in (13) can be obtained by considering the equivalent extended representation $\widetilde{W}(11)$,

$$
D \widetilde{W}[\delta \boldsymbol{\phi}]=\partial_{\boldsymbol{C}} \widetilde{W}: D \boldsymbol{C}[\delta \boldsymbol{\phi}]+\partial_{\boldsymbol{G}} \widetilde{W}: D \boldsymbol{G}[\delta \boldsymbol{\phi}]+\partial_{C} \widetilde{W} D C[\delta \boldsymbol{\phi}] ; \quad D \widetilde{W}[\delta \theta]=\partial_{\theta} \widetilde{W} \delta \theta .
$$

From (12), the directional derivatives of $\{\boldsymbol{C}, \boldsymbol{G}, C\}$, featuring in (15), can be expressed as

$$
D \boldsymbol{C}[\delta \boldsymbol{\phi}]=\left(\boldsymbol{\nabla}_{0} \delta \boldsymbol{\phi}\right)^{T} \boldsymbol{F}+\boldsymbol{F}^{T} \boldsymbol{\nabla}_{0} \delta \boldsymbol{\phi} ; \quad D \boldsymbol{G}[\delta \boldsymbol{\phi}]=\boldsymbol{C} \times D \boldsymbol{C}[\delta \boldsymbol{\phi}] ; \quad D C[\delta \boldsymbol{\phi}]=\boldsymbol{G}: D \boldsymbol{C}[\delta \boldsymbol{\phi}] .
$$

Finally, inserting (16) into (15) $)_{a}$ and comparison with (13) enables to obtain an equivalent expression for $\boldsymbol{S}$ and $\eta$ to those in equations (14) as

$$
\boldsymbol{S}=2 \partial_{\boldsymbol{C}} \widetilde{W}+2 \partial_{\boldsymbol{G}} \widetilde{W} \times \boldsymbol{C}+2 \partial_{C} \widetilde{W} \boldsymbol{G} ; \quad \eta=-\partial_{\theta} \widetilde{W} .
$$

\subsubsection{Additive split of the Helmholtz free energy}

Following calorimetry principles (refer to Appendix D), the Helmholtz energy can be formulated through an additive decomposition as

$$
\begin{aligned}
& W(\boldsymbol{F}, \boldsymbol{H}, J, \theta)=W_{m}(\boldsymbol{F}, \boldsymbol{H}, J)+W_{\theta}(\theta)-\underbrace{\left(\theta-\theta_{R}\right) \eta_{R}(J)}_{-W_{c}(J, \theta)} ; \\
& \widetilde{W}(\boldsymbol{C}, \boldsymbol{G}, C, \theta)=\widetilde{W}_{m}(\boldsymbol{C}, \boldsymbol{G}, C)+\widetilde{W}_{\theta}(\theta)-\underbrace{\left(\theta-\theta_{R}\right) \widetilde{\eta}_{R}(C)}_{-\widetilde{W}_{c}(C, \theta)},
\end{aligned}
$$

where $\theta_{R}$ denotes the reference temperature and $\eta_{R}(J)$ (or $\widetilde{\eta}_{R}(C)$ ) is a function that measures the entropy generated through deformation at the reference temperature $\theta_{R}{ }^{5}$. An example of mechanical contribution complying with (18) is the Mooney-Rivlin model $W_{M R}(\boldsymbol{F}, \boldsymbol{H}, J)$ $\left(\widetilde{W}_{M R}(\boldsymbol{C}, \boldsymbol{G}, C)\right)$, defined as

$$
\begin{aligned}
& W_{m}(\boldsymbol{F}, \boldsymbol{H}, J)=W_{M R}(\boldsymbol{F}, \boldsymbol{H}, J)=\frac{\mu_{1}}{2} I I_{\boldsymbol{F}}+\frac{\mu_{2}}{2} I_{\boldsymbol{H}}-\left(\mu_{1}+2 \mu_{2}\right) \ln J+\frac{\lambda}{2}(J-1)^{2} ; \\
& \widetilde{W}_{m}(\boldsymbol{C}, \boldsymbol{G}, C)=\widetilde{W}_{M R}(\boldsymbol{C}, \boldsymbol{G}, C)=\frac{\mu_{1}}{2} \operatorname{tr} \boldsymbol{C}+\frac{\mu_{2}}{2} \operatorname{tr} \boldsymbol{G}-\left(\mu_{1}+2 \mu_{2}\right) \ln C^{1 / 2}+\frac{\lambda}{2}\left(C^{1 / 2}-1\right)^{2},
\end{aligned}
$$

where $I I_{\boldsymbol{A}}=\boldsymbol{A}: \boldsymbol{A}$ and $\left\{\mu_{1}, \mu_{2}, \lambda\right\}$ are positive material parameters related to the shear and bulk moduli of the material in the origin (when $\boldsymbol{\nabla}_{0} \boldsymbol{\phi}=\boldsymbol{I}$ and $\theta=\theta_{R}$ ), namely $\left\{\mu_{R}, \lambda_{R}\right\}$ as

$$
\mu_{R}=\mu_{1}+\mu_{2} ; \quad \lambda_{R}=\lambda+2 \mu_{2} .
$$

The purely thermal contribution in (18) is defined as (see [17] and Appendix D)

$$
W_{\theta}(\theta)=\widetilde{W}_{\theta}(\theta)=c_{v}\left(\theta-\theta_{R}-\theta \ln \frac{\theta}{\theta_{R}}\right)
$$

where $c_{v} \geq 0$ is the specific heat capacity. A very typical expression adopted for the reference entropy function $\eta_{R}(J)$ (and $\widetilde{\eta}_{R}(C)$ ) is that of a Mie-Gruneisen model, defined as

$$
\eta_{R}(J)=c_{v} \Gamma_{0}\left\{\begin{array}{ll}
\frac{J^{q}-1}{q} & \text { if } q>0 ; \\
\ln J & \text { if } q=0 .
\end{array} \quad \widetilde{\eta}_{R}(C)=c_{v} \Gamma_{0} \begin{cases}\frac{C^{\frac{q}{2}}-1}{q} & \text { if } q>0 \\
\ln C^{1 / 2} & \text { if } q=0\end{cases}\right.
$$

\footnotetext{
${ }^{5}$ In this case, we have assumed that the reference entropy function is only dependent upon the volumetric deformation.
} 
with $q \geq 0$ a material parameter and $\Gamma_{0}$ a material parameter. For an additively decomposed mechanical contribution such as that in (19), the inequalities (9) become

$$
\begin{array}{rlrl}
g_{\boldsymbol{F}} & :=\delta \boldsymbol{F}: \partial_{\boldsymbol{F} \boldsymbol{F}}^{2} W_{m_{\boldsymbol{F}}}: \delta \boldsymbol{F} \geq 0 ; & g_{\boldsymbol{H}}:=\delta \boldsymbol{H}: \partial_{\boldsymbol{H} \boldsymbol{H}}^{2} W_{m_{\boldsymbol{H}}}: \delta \boldsymbol{H} \geq 0 \\
g_{J}:=W_{m_{J}}^{\prime \prime}-\left(\theta-\theta_{0}\right) \eta_{0}^{\prime \prime} \geq 0 ; & g_{\theta}:=W_{\theta}^{\prime \prime} \leq 0,
\end{array}
$$

which, for the model under consideration, lead to

$$
\begin{aligned}
& g_{\boldsymbol{F}}=\mu_{1} I I_{\delta \boldsymbol{F}} \\
& g_{\boldsymbol{H}}=\mu_{2} I I_{\delta \boldsymbol{H}} \\
& g_{J}= \begin{cases}\frac{\mu_{1}+2 \mu_{2}}{J^{2}}+\lambda-c_{v} \Gamma_{0}\left(\theta-\theta_{R}\right)(q-1) J^{q-2} & \text { if } q>0 ; \\
\frac{\mu_{1}+2 \mu_{2}}{J^{2}}+\lambda-c_{v} \Gamma_{0} \frac{\left(\theta-\theta_{R}\right)}{J^{2}} & \text { if } q=0 ;\end{cases} \\
& g_{\theta}=-\frac{c_{v}}{\theta} .
\end{aligned}
$$

Clearly, the only term in (24) which might compromise stability (inequalities (23)) is $g_{J}$. For materials where volumetric deformations are small (i.e. $J \approx 1$ ), the critical temperature $\theta_{c r}$ violating (23) can be estimated as

$$
\theta_{c r}=\theta_{R}\left(1-\frac{\gamma}{\theta_{R}}\right) ; \quad \gamma= \begin{cases}\frac{\mu_{1}+2 \mu_{2}+\lambda}{c_{v} \Gamma_{0}|(q-1)|} & \text { if } q>0 \\ \frac{\mu_{1}+2 \mu_{2}+\lambda}{c_{v} \Gamma_{0}} & \text { if } q=0 .\end{cases}
$$

It is possible to define the total Helmholtz functional $W(\boldsymbol{F}, \boldsymbol{H}, J)$ in (18) complying with the material stability condition in (9) unconditionally, namely, for the entire range of deformations and temperature values [12]. In order to do this, the mechanical contribution $W_{m}$ in (19) is carefully modified as

$$
\begin{aligned}
& W_{m}(\boldsymbol{F}, \boldsymbol{H}, J)=W_{M R}(\boldsymbol{F}, \boldsymbol{H}, J)+\theta_{R} c_{v} \Gamma_{0}(J-1)-\theta_{R} \eta_{R}(J) ; \\
& \widetilde{W}_{m}(\boldsymbol{C}, \boldsymbol{G}, C)=\widetilde{W}_{M R}(\boldsymbol{C}, \boldsymbol{G}, C)+\theta_{R} c_{v} \Gamma_{0}\left(C^{1 / 2}-1\right)-\theta_{R} \widetilde{\eta}_{R}(C) .
\end{aligned}
$$

Notice that the second term on both right-hand sides of (26) has been added in order to satisfy the stress-free condition for $W_{m}(\boldsymbol{F}, \boldsymbol{H}, J)$ (and $\widetilde{W}_{m}(\boldsymbol{C}, \boldsymbol{G}, C)$ ), namely (refer to equation $(17)_{a}$ )

$$
\left.\left(2 \partial_{\boldsymbol{C}} \widetilde{W}_{m}+2 \partial_{\boldsymbol{G}} \widetilde{W}_{m} \times \boldsymbol{C}+2 \partial_{C} \widetilde{W}_{m} \boldsymbol{G}\right)\right|_{\substack{\boldsymbol{C}=I \\ G=I \\ C=1}}=\mathbf{0}
$$

Furthermore, notice that a sufficient condition for the satisfaction of the material stability condition in (9) is the concavity of the entropy function $\eta_{R}(J)$ with respect to the Jacobian term $J$ (i.e. $\partial_{J J} \eta_{R} \leq 0$ ), satisfied by the expressions in equation $(22)_{a}$.

The constitutive model presented throughout this section has been included as an example. It is not essential for the time integrator that will be presented in Section 5.1 and alternative multiplicative split-based thermo-mechanical models can also be used.

\subsubsection{The internal energy}

Satisfaction of condition $(9)_{b}$ enables to establish a one-to-one and invertible relationship between the Helmholtz free energy functional $\widetilde{W}(\boldsymbol{C}, \boldsymbol{G}, C, \theta)$ and the internal energy functional $\widetilde{U}(\boldsymbol{C}, \boldsymbol{G}, C, \eta)$ by means of a partial Legendre transform as

$$
\widetilde{U}(\boldsymbol{C}, \boldsymbol{G}, C, \eta)=\max _{\theta}\{\theta \eta+\widetilde{W}(\boldsymbol{C}, \boldsymbol{G}, C, \theta)\} .
$$

From the internal energy it is possible to obtain the second Piola-Kirchhoff stress tensor and the temperature as

$$
\boldsymbol{S}=2 \partial_{\boldsymbol{C}} \widetilde{U}+2 \partial_{\boldsymbol{G}} \widetilde{U} \times \boldsymbol{C}+2 \partial_{C} \widetilde{U} \boldsymbol{G} ; \quad \theta=\partial_{\eta} \widetilde{U}
$$


From equation $(17)_{b}$ it is possible to obtain an explicit expression of $\theta$ as a function of $\eta$ for the specific thermo-mechanical model in (21) and (22) as

$$
\eta=:-\partial_{\theta} \widetilde{W}=\left(\widetilde{\eta}_{R}(C)+c_{v} \ln \frac{\theta}{\theta_{R}}\right) \rightarrow \theta=\theta_{R} \exp \left(\frac{\eta-\widetilde{\eta}_{R}(C)}{c_{v}}\right) .
$$

Introduction of (30) into (28) enables to obtain the following additive decomposition of the internal energy functional

$$
\widetilde{U}(\boldsymbol{C}, \boldsymbol{G}, C, \eta)=\widetilde{U}_{m}(\boldsymbol{C}, \boldsymbol{G}, C)+\widetilde{U}_{c}(C, \eta),
$$

with

$$
\widetilde{U}_{m}(\boldsymbol{C}, \boldsymbol{G}, C)=\widetilde{W}_{m}(\boldsymbol{C}, \boldsymbol{G}, C) ; \quad \widetilde{U}_{c}(C, \eta)=\theta_{R}\left(\kappa\left(\exp \left(\frac{\eta-\widetilde{\eta}_{R}(C)}{c_{v}}\right)-1\right)+\widetilde{\eta}_{R}(C)\right) .
$$

\subsection{Fourier law}

Fourier law relates the spatial heat flux $\boldsymbol{q}$ and the spatial gradient of $\theta$ by virtue of the following expression

$$
\boldsymbol{q}=-\boldsymbol{k} \boldsymbol{\nabla} \theta
$$

where $\boldsymbol{k}$ represents the semi-positive definite second order thermal conductivity tensor in the deformed configuration. As customary in continuum mechanics, the relation between $\boldsymbol{q}$ and its material counterpart $\boldsymbol{Q}$ featuring in equation (6) can be carried out by making use of the Gauss' theorem and the Nanson's rule (i.e. $d \boldsymbol{a}=\boldsymbol{H} d \boldsymbol{A}$ ) as

$$
\int_{\mathcal{B}} \operatorname{div} \boldsymbol{q} d v=\int_{\partial \mathcal{B}} \boldsymbol{q} \cdot d \boldsymbol{a}=\int_{\partial \mathcal{B}_{0}} \boldsymbol{Q} \cdot d \boldsymbol{A},
$$

with

$$
\boldsymbol{Q}=\boldsymbol{H}^{T} \boldsymbol{q}=-\boldsymbol{H}^{T} \boldsymbol{k} \boldsymbol{\nabla} \theta
$$

The spatial gradient of $\theta(35)$ can be conveniently related to its material counterpart as

$$
\boldsymbol{\nabla} \theta=\boldsymbol{F}^{-T} \boldsymbol{\nabla}_{0} \theta=J^{-1} \boldsymbol{H} \boldsymbol{\nabla}_{0} \theta .
$$

Finally, introduction of (36) into (35) yields

$$
\boldsymbol{Q}=-\boldsymbol{K} \nabla_{0} \theta ; \quad \boldsymbol{K}=J^{-1} \boldsymbol{H}^{T} \boldsymbol{k} \boldsymbol{H}
$$

\section{Thermo-Elastodynamics}

The objective of this section is to present the variational formulation that will be used in order to develop an EM time integration scheme in Section 5.

\subsection{Continuum formulation}

In order to derive a suitable EM time integrator, we first study the conservation properties of a thermo-elastic continuum controlled by the governing equations (4) and (6). For this, we present the following three weak form equations:

$$
\begin{aligned}
& \mathcal{W}_{\boldsymbol{v}}=\int_{\mathcal{B}_{0}}(\boldsymbol{v}-\dot{\boldsymbol{\phi}}) \cdot \rho_{0} \boldsymbol{w}_{\boldsymbol{v}} d V=0 ; \\
& \mathcal{W}_{\boldsymbol{\phi}}=\int_{\mathcal{B}_{0}} \rho_{0} \dot{\boldsymbol{v}} \cdot \boldsymbol{w}_{\boldsymbol{\phi}} d V+\int_{\mathcal{B}_{0}} \boldsymbol{S}: \frac{1}{2} D \boldsymbol{C}\left[\boldsymbol{w}_{\boldsymbol{\phi}}\right] d V-\int_{\mathcal{B}_{0}} \boldsymbol{f}_{0} \cdot \boldsymbol{w}_{\boldsymbol{\phi}} d V-\int_{\partial_{t} \mathcal{B}_{0}} \boldsymbol{t}_{0} \cdot \boldsymbol{w}_{\boldsymbol{\phi}} d A=0 ; \\
& \mathcal{W}_{\theta}=\int_{\mathcal{B}_{0}} \frac{d}{d t}(\theta \eta) w_{\theta} d V-\int_{\mathcal{B}_{0}} \dot{\theta} \eta w_{\theta} d V-\int_{\mathcal{B}_{0}} \boldsymbol{Q} \cdot \nabla_{0} w_{\theta} d V-\int_{\mathcal{B}_{0}} R_{\theta} w_{\theta} d V-\int_{\partial_{Q} \mathcal{B}_{0}} Q_{\theta} w_{\theta} d A=0,
\end{aligned}
$$


where $\{\boldsymbol{v}, \boldsymbol{\phi}, \theta\} \in \mathbb{V}^{\phi} \times \mathbb{V}^{\phi} \times \mathbb{V}^{\theta}$ and $\left\{\boldsymbol{w}_{\boldsymbol{v}}, \boldsymbol{w}_{\phi}, w_{\theta}\right\} \in \mathbb{V}_{0}^{\phi} \times \mathbb{V}_{0}^{\phi} \times \mathbb{V}_{0}^{\theta 6}$, with

$$
\begin{array}{rlrl}
\mathbb{V}^{\phi} & =\left\{\phi: \mathcal{B}_{0} \rightarrow \mathbb{R}^{3} ; \quad(\boldsymbol{\phi})_{i} \in H^{1}\left(\mathcal{B}_{0}\right)\right\} ; & \mathbb{V}^{\theta}=\left\{\theta: \mathcal{B}_{0} \rightarrow \mathbb{R} ; \quad \theta \in H^{1}\left(\mathcal{B}_{0}\right)\right\} ; \\
\mathbb{V}_{0}^{\phi}=\left\{\forall \boldsymbol{\phi} \in \mathbb{V}^{\phi} ; \quad \boldsymbol{\phi}=\mathbf{0} \text { on } \partial_{\phi} \mathcal{B}_{0}\right\} ; & \mathbb{V}_{0}^{\theta}=\left\{\forall \theta \in \mathbb{V}^{\theta} ; \quad \theta=0 \text { on } \partial_{\theta} \mathcal{B}_{0}\right\} .
\end{array}
$$

\subsection{Balance laws and integrals in thermo-elastodynamics}

Starting with the stationary conditions (38) the following sections derive the global conservation laws that govern the motion of the thermo-elastic continuum.

\subsubsection{Global form for conservation of linear momentum}

For a displacement field $\boldsymbol{w}_{\boldsymbol{\phi}}=\boldsymbol{\xi}$, with $\mathbb{R}^{3} \ni \boldsymbol{\xi}=$ const., the stationary condition in $(38)_{b}$ leads to the global form of the conservation of linear momentum, namely

$$
\dot{\boldsymbol{L}}-\boldsymbol{F}^{\mathrm{ext}}=\mathbf{0} ; \quad \boldsymbol{L}=\int_{\mathcal{B}_{0}} \rho_{0} \boldsymbol{v} d V ; \quad \boldsymbol{F}^{\mathrm{ext}}=\int_{\partial_{\boldsymbol{t}} \mathcal{B}_{0}} \boldsymbol{t}_{0} d A+\int_{\mathcal{B}_{0}} \boldsymbol{f}_{0} d V
$$

where $\boldsymbol{L}$ represents the total linear momentum and $\boldsymbol{F}^{\text {ext }}$, the total external force. From (40) it is possible to conclude that $\boldsymbol{L}$ is a constant of motion for the case of vanishing external forces $\boldsymbol{F}^{\mathrm{ext}}$. Nonetheless, notice that a translational field is potentially incompatible with the presence of Dirichlet boundary conditions. This entails that above result is only valid for the case where Dirichlet boundary conditions are not applied. Otherwise, the global balance in equation (40) must account for the reaction forces induced in the boundary $\partial_{\phi} \mathcal{B}_{0}$ as a result of the application of the Dirichlet boundary conditions. However, for the sake of simplicity and brevity of exposition, we will not include the effect of mechanical or thermal boundary conditions in the forthcoming balance laws.

\subsubsection{Global form for conservation of angular momentum}

For a rotational field $\boldsymbol{w}_{\boldsymbol{\phi}}=\boldsymbol{\xi} \times \boldsymbol{\phi}$, with $\mathbb{R}^{3} \ni \boldsymbol{\xi}=$ const., the stationary condition in $(38)_{b}$ leads to the global form of the conservation of angular momentum, namely

$$
\dot{\boldsymbol{J}}-\boldsymbol{M}^{\mathrm{ext}}=\mathbf{0} ; \quad \boldsymbol{J}=\int_{\mathcal{B}_{0}} \boldsymbol{\phi} \times \rho_{0} \boldsymbol{v} d V ; \quad \boldsymbol{M}^{\mathrm{ext}}=\int_{\partial_{\boldsymbol{t}} \mathcal{B}_{0}} \boldsymbol{\phi} \times \boldsymbol{t}_{0} d A+\int_{\mathcal{B}_{0}} \boldsymbol{\phi} \times \boldsymbol{f}_{0} d V,
$$

where $\boldsymbol{J}$ represents the total angular momentum and $\boldsymbol{M}^{\text {ext }}$, the total external torque. From (41), it is clear that $\boldsymbol{J}$ is a constant of motion for vanishing external torques $\boldsymbol{M}^{\text {ext }}$.

\subsubsection{Global form for conservation of power}

Let us replace the test functions $\left\{\boldsymbol{w}_{\boldsymbol{v}}, \boldsymbol{w}_{\boldsymbol{\phi}}\right\}$ in (38) with $\{\dot{\boldsymbol{v}}, \dot{\boldsymbol{\phi}}\} \in\left\{\mathbb{V}_{0}^{\phi} \times \mathbb{V}_{0}^{\phi}\right\}$ and let us take $w_{\theta}=1$. This yields

$$
\begin{aligned}
& \int_{\mathcal{B}_{0}}(\boldsymbol{v}-\dot{\boldsymbol{\phi}}) \cdot \rho_{0} \dot{\boldsymbol{v}} d V=0 ; \\
& \int_{\mathcal{B}_{0}} \rho_{0} \dot{\boldsymbol{v}} \cdot \dot{\boldsymbol{\phi}} d V+\int_{\mathcal{B}_{0}} \boldsymbol{S}: \frac{1}{2} \dot{\boldsymbol{C}} d V-\int_{\mathcal{B}_{0}} \boldsymbol{f}_{0} \cdot \dot{\boldsymbol{\phi}} d V-\int_{\partial_{\boldsymbol{t}} \mathcal{B}_{0}} \boldsymbol{t}_{0} \cdot \dot{\boldsymbol{\phi}} d A=0 ; \\
& \int_{\mathcal{B}_{0}} \frac{d}{d t}(\theta \eta) d V-\int_{\mathcal{B}_{0}} \dot{\theta} \eta d V-\int_{\mathcal{B}_{0}} R_{\theta} d V-\int_{\partial_{Q} \mathcal{B}_{0}} Q_{\theta} d A=0 .
\end{aligned}
$$

\footnotetext{
${ }^{6}$ Notice in that $\phi$ must satisfy in addition the condition $J>0$ a.e.
} 
Addition of the three equations in (42) leads, in the case of time independent forces $\boldsymbol{f}_{0}$ and $\boldsymbol{t}_{0}$ to

$$
\dot{K}+\int_{\mathcal{B}_{0}}\left(\boldsymbol{S}: \frac{1}{2} \dot{\boldsymbol{C}}-\dot{\theta} \eta\right) d V+\int_{\mathcal{B}_{0}} \frac{d}{d t}(\theta \eta) d V-\dot{\Pi}_{\mathrm{ext}}(\boldsymbol{\phi})-\dot{\mathcal{Q}}_{\mathrm{ext}}=0
$$

where $\dot{K}, \dot{\Pi}_{\text {ext }}$ and $\dot{\mathcal{Q}}_{\text {ext }}$ represent the kinetic power, the external mechanical power and the external heat power, defined as

$$
\dot{K}=\int_{\mathcal{B}_{0}} \rho_{0} \boldsymbol{v} \cdot \dot{\boldsymbol{v}} d V ; \quad \dot{\Pi}_{\mathrm{ext}}=\int_{\mathcal{B}_{0}} \boldsymbol{f}_{0} \cdot \dot{\boldsymbol{\phi}} d V+\int_{\partial_{\boldsymbol{t}} \mathcal{B}_{0}} \boldsymbol{t}_{0} \cdot \dot{\boldsymbol{\phi}} d A ; \quad \dot{\mathcal{Q}}_{\mathrm{ext}}=\int_{\mathcal{B}_{0}} R_{\theta} d V+\int_{\partial_{Q} \mathcal{B}_{0}} Q_{\theta} d A
$$

Making use of (15) and (17) in (43) yields

$$
\dot{K}+\int_{\mathcal{B}_{0}} \dot{\widetilde{W}}(\boldsymbol{C}, \boldsymbol{G}, C, \theta) d V+\int_{\mathcal{B}_{0}} \frac{d}{d t}(\theta \eta) d V-\dot{\Pi}_{\text {ext }}(\boldsymbol{\phi})-\dot{\mathcal{Q}}_{\text {ext }}=0 .
$$

Making use of the Legendre transformation in (28) it is possible to re-express (45) as

$$
\dot{K}+\int_{\mathcal{B}_{0}} \dot{\widetilde{U}}(\boldsymbol{C}, \boldsymbol{G}, C, \eta) d V-\dot{\Pi}_{\mathrm{ext}}(\boldsymbol{\phi})-\dot{\mathcal{Q}}_{\mathrm{ext}}=0 .
$$

Therefore, for vanishing external mechanical and thermal power, the following condition holds,

$$
\dot{\mathcal{H}}=0 ; \quad \mathcal{H}=K+\int_{\mathcal{B}_{0}} \widetilde{U}(\boldsymbol{C}, \boldsymbol{G}, C, \eta) d V
$$

and accordingly, the Hamiltonian $\mathcal{H}$ or total energy is conserved throughout the motion of the continuum.

\section{Energy-Momentum integration scheme for thermo-elastodynamics}

Following the work of Simo [31], Gonzalez [18], Romero [28] and Betsch [7, 6] in the context of nonlinear elasticity and Franke et al. [17] in the context of thermoelasticity, the objective of this section is to propose an EM preserving time discretisation scheme for the weak forms in (38).

\subsection{Design of the EM scheme}

Let us consider a sequence of time steps $\left\{t_{1}, t_{2}, \ldots, t_{n}, t_{n+1}\right\}$, where $t_{n+1}$ denotes the current time step. From the weak forms in (38), the following implicit one-step time integrator is proposed

$$
\begin{aligned}
\left(\mathcal{W}_{\boldsymbol{v}}\right)_{\text {algo }} & =\int_{\mathcal{B}_{0}}\left(\boldsymbol{v}_{n+1 / 2}-\frac{\Delta \boldsymbol{\phi}}{\Delta t}\right) \cdot \rho_{0} \boldsymbol{w}_{\boldsymbol{v}} d V=0 ; \\
\left(\mathcal{W}_{\boldsymbol{\phi}}\right)_{\text {algo }} & =\int_{\mathcal{B}_{0}} \rho_{0} \frac{\Delta \boldsymbol{v}}{\Delta t} \cdot \boldsymbol{w}_{\boldsymbol{\phi}} d V+\int_{\mathcal{B}_{0}}\left(\boldsymbol{S}: \frac{1}{2} D \boldsymbol{C}\left[\boldsymbol{w}_{\phi}\right]\right)_{\text {algo }} d V-\int_{\mathcal{B}_{0}} \boldsymbol{f}_{0_{n+1 / 2}} \cdot \boldsymbol{w}_{\boldsymbol{\phi}} d V \\
& -\int_{\partial_{\boldsymbol{t}} \mathcal{B}_{0}} \boldsymbol{t}_{0_{n+1 / 2}} \cdot \boldsymbol{w}_{\boldsymbol{\phi}} d A=0 \\
\left(\mathcal{W}_{\theta}\right)_{\text {algo }}= & \int_{\mathcal{B}_{0}} \frac{\Delta(\theta \eta)}{\Delta t} w_{\theta} d V-\int_{\mathcal{B}_{0}} \frac{\Delta \theta}{\Delta t} \eta_{\text {algo }} w_{\theta} d V-\int_{\mathcal{B}_{0}} \boldsymbol{Q}_{n+1 / 2} \cdot \nabla_{0} w_{\theta} d V \\
& -\int_{\mathcal{B}_{0}} R_{\theta n+1 / 2} w_{\theta} d V-\int_{\partial_{Q} \mathcal{B}_{0}} Q_{\theta_{n+1 / 2}} w_{\theta} d A=0 .
\end{aligned}
$$

Note that $\left(\mathcal{W}_{\boldsymbol{v}}\right)_{\text {algo }},\left(\mathcal{W}_{\boldsymbol{\phi}}\right)_{\text {algo }}$ and $\left(\mathcal{W}_{\theta}\right)_{\text {algo }}$ in $(48)$ represent the algorithmic or time discrete versions of the stationary conditions in $(38)$ and $(\bullet)_{n+1 / 2}=\frac{1}{2}\left((\bullet)_{n+1}+(\bullet)_{n}\right)$ and $\Delta(\bullet)=(\bullet)_{n+1}-$ 
$(\bullet)_{n}$. Following the work of Betsch. et. al [7, 17, 27], we draw a parallelism between the terms $\left\{\left(\boldsymbol{S}: \frac{1}{2} D \boldsymbol{C}\left[\boldsymbol{w}_{\phi}\right]\right)_{\text {algo }}, \eta_{\text {algo }}\right\}$ and the expressions for $\{D \widetilde{W}[\delta \boldsymbol{\phi}], \eta\}$ in $(15)_{a}$ and $(17)_{b}$ and hence, we advocate for an analogous expression of the algorithmic terms as

$$
\begin{aligned}
\left(\boldsymbol{S}: \frac{1}{2} D \boldsymbol{C}\left[\boldsymbol{w}_{\phi}\right]\right)_{\mathrm{algo}} & =D_{\boldsymbol{C}} \widetilde{W}:\left(D \boldsymbol{C}\left[\boldsymbol{w}_{\phi}\right]\right)_{\mathrm{algo}}+D_{\boldsymbol{G}} \widetilde{W}:\left(D \boldsymbol{G}\left[\boldsymbol{w}_{\phi}\right]\right)_{\mathrm{algo}}+D_{C} \widetilde{W}\left(D C\left[\boldsymbol{w}_{\phi}\right]\right)_{\mathrm{algo}} ; \\
\eta_{\mathrm{algo}} & =-D_{\theta} \widetilde{W},
\end{aligned}
$$

with the algorithmic directional derivatives $\left\{\left(D \boldsymbol{C}\left[\boldsymbol{w}_{\phi}\right]\right)_{\text {algo }},\left(D \boldsymbol{G}\left[\boldsymbol{w}_{\phi}\right]\right)_{\text {algo }},\left(D C\left[\boldsymbol{w}_{\phi}\right]\right)_{\text {algo }}\right\}$ defined as in Reference [27], i.e.

$$
\begin{array}{lll}
\left(D \boldsymbol{C}\left[\boldsymbol{w}_{\boldsymbol{\phi}}\right]\right)_{\text {algo }}=\left(\left(\boldsymbol{\nabla}_{0} \boldsymbol{w}_{\boldsymbol{\phi}}\right)^{T} \boldsymbol{F}_{n+1 / 2}+\boldsymbol{F}_{n+1 / 2}^{T} \boldsymbol{\nabla}_{0} \boldsymbol{w}_{\boldsymbol{\phi}}\right) ; & \\
\left(D \boldsymbol{G}\left[\boldsymbol{w}_{\boldsymbol{\phi}}\right]\right)_{\text {algo }}=\boldsymbol{C}_{\text {algo }} \times\left(D \boldsymbol{C}\left[\boldsymbol{w}_{\boldsymbol{\phi}}\right]\right)_{\mathrm{algo}} ; & \boldsymbol{C}_{\text {algo }}=\boldsymbol{C}_{n+1 / 2} ; \\
\left(D C\left[\boldsymbol{w}_{\boldsymbol{\phi}}\right]\right)_{\mathrm{algo}}=\boldsymbol{G}_{\text {algo }}:\left(D \boldsymbol{C}\left[\boldsymbol{w}_{\boldsymbol{\phi}}\right]\right)_{\mathrm{algo}} ; & \boldsymbol{G}_{\text {algo }}=\frac{1}{3}\left(\boldsymbol{C}_{n+1 / 2} \times \boldsymbol{C}_{n+1 / 2}+\boldsymbol{G}_{n+1 / 2}\right) .
\end{array}
$$

In (49), $\left\{D_{C} \widetilde{W}, D_{G} \widetilde{W}, D_{C} \widetilde{W}, D_{\theta} \widetilde{W}\right\}$ represent the discrete derivatives (cf. [18, 17]) of the Helmholtz energy $\widetilde{W}$ with respect to $\{\boldsymbol{C}, \boldsymbol{G}, C, \theta\}$, respectively, which are the algorithmic or time discrete counterparts of $\left\{\partial_{\boldsymbol{C}} \widetilde{W}, \partial_{\boldsymbol{G}} \widetilde{W}, \partial_{C} \widetilde{W}, \partial_{\theta} \widetilde{W}\right\}$, respectively. Finally, introduction of (50) into (49) permits to conveniently re-write (49) as

$$
\left(\boldsymbol{S}: \frac{1}{2} D \boldsymbol{C}\left[\boldsymbol{w}_{\phi}\right]\right)_{\text {algo }}=\boldsymbol{S}_{\text {algo }}: \frac{1}{2}\left(D \boldsymbol{C}\left[\boldsymbol{w}_{\phi}\right]\right)_{\text {algo }} ; \quad \boldsymbol{S}_{\text {algo }}=2 D_{\boldsymbol{C}} \widetilde{W}+2 D_{\boldsymbol{G}} \widetilde{W} \times \boldsymbol{C}_{\text {algo }}+2 D_{C} \widetilde{W} \boldsymbol{G}_{\text {algo }} .
$$

Remark 1. It is important to emphasise that the derivation of the EM time integrator in (48), associated with the set of weak forms in (38), is based on the following four steps, which are common for the design of any EM time integrator, namely:

1. Substitution of time rates $(\bullet)$ with $\frac{\Delta(\bullet)}{\Delta t}$, where $\Delta(\bullet)=(\bullet)_{n+1}-(\bullet)_{n}$.

2. Midpoint evaluation of terms, namely $(\bullet)_{n+1 / 2}=\frac{1}{2}\left((\bullet)_{n+1}+(\bullet)_{n}\right)$.

3. Substitution of the derivatives of the Helmholtz energy functional $\left\{\partial_{\boldsymbol{C}} \widetilde{W}, \partial_{\boldsymbol{G}} \widetilde{W}, \partial_{C} \widetilde{W}, \partial_{\theta} \widetilde{W}\right\}$ with their discrete counterparts $\left\{D_{\boldsymbol{C}} \widetilde{W}, D_{G} \widetilde{W}, D_{C} \widetilde{W}, D_{\theta} \widetilde{W}\right\}$.

4. Replacement of the directional derivatives of the symmetric strain measures $\{\boldsymbol{C}, \boldsymbol{G}, C\}$, namely $\left\{D \boldsymbol{C}\left[\boldsymbol{w}_{\phi}\right], D \boldsymbol{G}\left[\boldsymbol{w}_{\phi}\right], D C\left[\boldsymbol{w}_{\phi}\right]\right\}$, with their carefully selected algorithmic counterparts, namely $\left\{\left(D \boldsymbol{C}\left[\boldsymbol{w}_{\phi}\right]\right)_{\text {algo }},\left(D \boldsymbol{G}\left[\boldsymbol{w}_{\phi}\right]\right)_{\text {algo }},\left(D C\left[\boldsymbol{w}_{\phi}\right]\right)_{\text {algo }}\right\}$.

Remark 2. Notice that both the exact and algorithmic entropy variables, namely $\eta$ and $\eta_{\text {algo }}$, respectively, feature in equation (48). The first (i.e. $\eta$, on the first term on the right hand side of (48)) is obtained according to equation (17) whereas the second, (i.e. $\eta_{\text {algo }}$, on the second term on the right hand side of (48)) is obtained according to equation $(49)_{b}$.

Remark 3. Comparison of the EM scheme proposed in equation (48) against the EM scheme in Reference [17] (and succinctly summarised in Appendix C) illustrates the simplicity of the new formulation with respect to the latter.

Remark 4. Notice that, as for any EM time integrator, discretisation and linearisation of the time integrator in (48) leads to an unsymmetric system of equations, in contrast to the midpoint rule. 


\subsubsection{Discretive derivatives of the internal energy}

We follow in this work a definition of the discrete derivatives $\left\{D_{C} \widetilde{W}, D_{G} \widetilde{W}, D_{C} \widetilde{W}, D_{\theta} \widetilde{W}\right\}$ of the Helmholtz free energy functional based on the derivation presented in [17] for energies depending upon several arguments. These generic expressions, presented in Appendix B, satisfy two crucial properties for the design of EM time integrators, namely:

- They fulfil the so called directionality property [17, 18],

$$
D_{\boldsymbol{C}} \widetilde{W}: \Delta \boldsymbol{C}+D_{\boldsymbol{G}} \widetilde{W}: \Delta \boldsymbol{G}+D_{C} \widetilde{W} \Delta C+D_{\theta} \widetilde{W} \Delta \theta=\Delta \widetilde{W}
$$

- They are well defined in the limit as $\|\Delta \boldsymbol{C}\| \rightarrow 0,\|\Delta \boldsymbol{G}\| \rightarrow 0,\|\Delta C\| \rightarrow 0$ and $\Delta \theta \rightarrow 0$.

The first property is critical for the algorithm in (48) to preserve the balance of power in (46) in the discrete setting. The second condition ensures that for sufficiently regular solutions

$$
\begin{array}{ll}
D_{C} \widetilde{W}=\partial_{C} \widetilde{W}\left(\widetilde{\mathcal{V}}_{n+1 / 2}\right)+O\left(\Delta t^{2}\right) ; & D_{G} \widetilde{W}=\partial_{G} \widetilde{W}\left(\widetilde{\mathcal{V}}_{n+1 / 2}\right)+O\left(\Delta t^{2}\right) \\
D_{C} \widetilde{W}=\partial_{C} \widetilde{W}\left(\widetilde{\mathcal{V}}_{n+1 / 2}\right)+O\left(\Delta t^{2}\right) ; & D_{\theta} \widetilde{W}=\partial_{\theta} \widetilde{W}\left(\widetilde{\mathcal{V}}_{n+1 / 2}\right)+O\left(\Delta t^{2}\right)
\end{array}
$$

with $\widetilde{\mathcal{V}}=\{\boldsymbol{C}, \boldsymbol{G}, C, \theta\}$, guaranteeing that the proposed EM time integrator is second order accurate. For the specific constitutive model in equations (21), (22) and (26), each of the discrete derivatives adopt the following extremely simple expressions

$$
\begin{aligned}
D_{C} \widetilde{W} & =\frac{\mu_{1}}{2} \boldsymbol{I} ; \\
D_{G} \widetilde{W} & =\frac{\mu_{2}}{2} \boldsymbol{I} ; \\
D_{C} \widetilde{W} & =\frac{\widetilde{W}_{m_{C}}\left(C_{n+1}\right)-\widetilde{W}_{m_{C}}\left(C_{n}\right)}{\Delta C} \\
& +\frac{1}{2} \frac{\widetilde{W_{c}}\left(C_{n+1}, \theta_{n}\right)-\widetilde{W}_{c}\left(C_{n}, \theta_{n}\right)}{\Delta C}+\frac{1}{2} \frac{\widetilde{W}_{c}\left(C_{n+1}, \theta_{n+1}\right)-\widetilde{W}_{c}\left(C_{n}, \theta_{n+1}\right)}{\Delta C} ; \\
D_{\theta} \widetilde{W} & =\frac{\widetilde{W_{\theta}}\left(\theta_{n+1}\right)-\widetilde{W}_{\theta}\left(\theta_{n}\right)}{\Delta \theta} \\
& +\frac{1}{2} \frac{\widetilde{W}_{c}\left(C_{n}, \theta_{n+1}\right)-\widetilde{W_{c}}\left(C_{n}, \theta_{n}\right)}{\Delta \theta}+\frac{1}{2} \frac{\widetilde{W}_{c}\left(C_{n+1}, \theta_{n+1}\right)-\widetilde{W}_{c}\left(C_{n+1}, \theta_{n}\right)}{\Delta \theta} .
\end{aligned}
$$

Notice the simplicity of the expressions of the multiple discretive derivatives with respect to those that would be obtained in the classical approach, whereby two very complex directional derivatives are needed, namely $\left\{D_{C} \widetilde{\Psi}, D_{\theta} \widetilde{\Psi}\right\}$ (cf. $\left.[17,25]\right)$.

Remark 5. Most authors (see Reference [21]) make use of $\eta_{\text {algo }}=\eta_{n+1 / 2}$ in (48) instead of our definition in $(49)_{b}$ involving the discrete derivative $D_{\theta} W$. In addition, they use $D_{\theta} W=$ $-\eta\left(\boldsymbol{C}_{n+1 / 2}, \theta_{n+1 / 2}\right)$ in the directionality property (52), which yields the alternative directionality property

$$
D_{\boldsymbol{C}} \widetilde{W}: \Delta \boldsymbol{C}+D_{\boldsymbol{G}} \widetilde{W}: \Delta \boldsymbol{G}+D_{C} \widetilde{W} \Delta C=\Delta \widetilde{W}+\eta\left(\boldsymbol{C}_{n+1 / 2}, \theta_{n+1 / 2}\right) \Delta \theta,
$$

where $\eta\left(\boldsymbol{C}_{n+1 / 2}, \theta_{n+1 / 2}\right)$ is evaluated according to $(17)_{b}$, i.e.

$$
\eta\left(\boldsymbol{C}_{n+1 / 2}, \theta_{n+1 / 2}\right)=-\left.\partial_{\theta} \widetilde{W}(\boldsymbol{C}, \theta)\right|_{\boldsymbol{C}=\boldsymbol{C}_{n+1 / 2}, \theta=\theta_{n+1 / 2}} .
$$

Notice that the directionality property (55) differs from that in (52) in two aspects: (a) the additional term $\eta_{n+1 / 2} \Delta \theta$, necessary to guarantee consistency (energy conservation); (b) the 
absence of the discrete derivative with respect to temperature $\left(D_{\theta} \widetilde{W}\right)$, in contrast to expression (52). The two contrasting aspects mentioned must have necessarily an impact on the definition of the discrete derivatives $\left\{D_{C} \widetilde{W}, D_{G} \widetilde{W}, D_{C} \widetilde{W}\right\}$. In particular, for the model described in equations (21), (22) and (26) (where the entropy depends upon the volumetric term $C$ ), making use of Appendix $\mathrm{B}$ and considering the extra term on the right hand side of (55) yields expressions for the discrete derivatives $D_{C} \widetilde{W}, D_{G} \widetilde{W}$ and $D_{C} \widetilde{W}$, differing with respect to those in (54) in the addition of three consistency restoring terms, namely

$$
\begin{aligned}
& D_{\boldsymbol{C}} \widetilde{W}=\mu_{1} \boldsymbol{I}+\underbrace{\alpha_{\boldsymbol{C}} \frac{\eta\left(C_{n+1 / 2}, \theta_{n+1 / 2}\right) \Delta \theta}{\|\Delta \boldsymbol{C}\|^{2}} \Delta \boldsymbol{C}}_{\text {Consistency restoring term }} ; \\
& D_{C} \widetilde{W}=\mu_{2} \boldsymbol{I}+\underbrace{\alpha_{\boldsymbol{G}} \frac{\eta\left(C_{n+1 / 2}, \theta_{n+1 / 2}\right) \Delta \theta}{\|\Delta \boldsymbol{G}\|^{2}} \Delta \boldsymbol{G}}_{\text {Consistency restoring term }} ; \\
& D_{C} \widetilde{W}=\frac{\widetilde{W}_{m_{C}}\left(C_{n+1}\right)-\widetilde{W}_{m_{C}}\left(C_{n}\right)}{\Delta C} \\
& +\frac{1}{2} \frac{\widetilde{W}_{c}\left(C_{n+1}, \theta_{n}\right)-\widetilde{W}_{c}\left(C_{n}, \theta_{n}\right)}{\Delta C}+\frac{1}{2} \frac{\widetilde{W}_{c}\left(C_{n+1}, \theta_{n+1}\right)-\widetilde{W}_{c}\left(C_{n}, \theta_{n+1}\right)}{\Delta C} \\
& +\underbrace{\alpha_{C} \frac{\eta\left(C_{n+1 / 2}, \theta_{n+1 / 2}\right) \Delta \theta}{\Delta C}}_{\text {Consistency restoring term }},
\end{aligned}
$$

where $\alpha_{\boldsymbol{C}}, \alpha_{\boldsymbol{G}}, \alpha_{C} \in \mathbb{R}$ must satisfy the relationship $\alpha_{\boldsymbol{C}}+\alpha_{\boldsymbol{G}}+\alpha_{C}=1$ in order to guarantee (55). Obviously, for the model considered in the paper, where the coupling between the thermal and mechanical part is dictated exclusively by the volumetric term $C$, a judicious choice of $\left\{\alpha_{\boldsymbol{C}}, \alpha_{\boldsymbol{G}}, \alpha_{C}\right\}$ yielding a simple expression for the discrete derivatives (57) would be

$$
\alpha_{\boldsymbol{C}}=0 ; \quad \alpha_{\boldsymbol{G}}=0 ; \quad \alpha_{C}=1 .
$$

However, for thermomechanical models where the coupling depends upon the tensor $\boldsymbol{C}$ (and not its determinant), such as in the case of modified entropic elasticity, a better choice of parameters could be

$$
\alpha_{\boldsymbol{C}}=1 ; \quad \alpha_{\boldsymbol{G}}=0 ; \quad \alpha_{C}=0 .
$$

Since the EM scheme presented in Section 5.1 does not need to incorporate consistency restoring terms in none of its four discrete derivatives, it is easy to be systematised and generalised regardless of the constitutive model used, and even extended to more complex multi-physics scenarios, such as that of thermo-electro-elasticity, without taking extra considerations regarding the suitability of the values for $\left\{\alpha_{\boldsymbol{C}}, \alpha_{\boldsymbol{G}}, \alpha_{C}\right\}$. Notice that the paper in Reference [17] succeded in the derivation of a EM scheme devoid from consistency restoring terms in the context of thermomechanics. However, we believe that the rearrangement of the conservation of energy equation in (6), in conjunction with the introduction of the algorithmic entropy $\eta_{\text {algo }}$ on the second term on the right hand side of (48) has resulted in a considerably simpler scheme than that in [17] (see Appendix C for a comparison between the EM scheme proposed and that in Reference [17]).

\subsection{Discrete form of the balance laws and integrals in thermo-elastodynamics}

A similar procedure to that in Section 4.2.1 will be followed in order to verify that the proposed time integration scheme presented (48) possesses the conservation properties as presented in Sections 4.2 .1 to 4.2 .3 . 


\subsubsection{Discrete form of the global form for conservation of linear momentum}

Following a similar procedure to that in Section 4.2.1, taking $\boldsymbol{w}_{\phi}=\boldsymbol{\xi}$, with $\mathbb{R}^{3} \ni \boldsymbol{\xi}=$ const. in $\mathcal{W}_{\phi}$ in $(48)_{b}$ yields

$$
\frac{\Delta \boldsymbol{L}}{\Delta t}-\boldsymbol{F}_{n+1 / 2}^{\mathrm{ext}}=\mathbf{0} ; \quad \boldsymbol{F}_{n+1 / 2}^{\mathrm{ext}}=\int_{\partial_{\boldsymbol{t}} \mathcal{B}_{0}} \boldsymbol{t}_{0_{n+1 / 2}} d A+\int_{\mathcal{B}_{0}} \boldsymbol{f}_{0_{n+1 / 2}} d V
$$

From equation (60) and for vanishing external forces $\boldsymbol{F}_{n+1 / 2}^{\text {ext }}$, it can be seen that the total linear momentum $\boldsymbol{L}$ remains constant.

\subsubsection{Discrete form of the global form for conservation of angular momentum}

Taking $\boldsymbol{w}_{\boldsymbol{\phi}}=\boldsymbol{\xi} \times \boldsymbol{\phi}_{n+1 / 2}$, with $\mathbb{R}^{3} \ni \boldsymbol{\xi}=$ const. in $\mathcal{W}_{\boldsymbol{\phi}}$ in $(48)_{b}$ yields

$$
\frac{\Delta \boldsymbol{J}}{\Delta t}-\boldsymbol{M}_{n+1 / 2}^{\mathrm{ext}}=\mathbf{0} ; \quad \boldsymbol{M}_{n+1 / 2}^{\mathrm{ext}}=\int_{\partial_{\boldsymbol{t}} \mathcal{B}_{0}} \boldsymbol{\phi}_{n+1 / 2} \times \boldsymbol{t}_{0_{n+1 / 2}} d A+\int_{\mathcal{B}_{0}} \boldsymbol{\phi}_{n+1 / 2} \times \boldsymbol{f}_{0_{n+1 / 2}} d V .
$$

From equation (61) and for vanishing external torques $\boldsymbol{M}_{n+1 / 2}^{\text {ext }}$, it can be seen that the total angular momentum $\boldsymbol{J}$ remains constant.

\subsubsection{Discrete form of the global form for the balance of power}

In this section, a similar analysis to that in Section (4.2.3) will be presented for the semidiscrete weak forms in (48). For this purpose, we replace in (48) the test functions $\left\{\boldsymbol{w}_{\boldsymbol{v}}, \boldsymbol{w}_{\boldsymbol{\phi}}\right\}$ with $\{\Delta \boldsymbol{v} / \Delta t, \Delta \phi / \Delta t\} \in \mathbb{V}_{0}^{\phi} \times \mathbb{V}_{0}^{\phi}$ and $w_{\theta}=1$. This yields

$$
\begin{aligned}
& \int_{\mathcal{B}_{0}}\left(\boldsymbol{v}_{n+1 / 2}-\frac{\Delta \boldsymbol{\phi}}{\Delta t}\right) \cdot \rho_{0} \frac{\Delta \boldsymbol{v}}{\Delta t} d V=0 ; \\
& \int_{\mathcal{B}_{0}} \rho_{0} \frac{\Delta \boldsymbol{v}}{\Delta t} \cdot \frac{\Delta \boldsymbol{\phi}}{\Delta t} d V+\int_{\mathcal{B}_{0}} \frac{1}{\Delta t} \boldsymbol{S}_{\text {algo }}: \frac{1}{2}\left(D \boldsymbol{C}\left[\boldsymbol{w}_{\phi}\right]\right)_{\text {algo }} d V-\int_{\mathcal{B}_{0}} \boldsymbol{f}_{0} \cdot \frac{\Delta \boldsymbol{\phi}}{\Delta t} d V-\int_{\partial_{t} \mathcal{B}_{0}} \boldsymbol{t}_{0} \cdot \frac{\Delta \boldsymbol{\phi}}{\Delta t} d A=0 ; \\
& \int_{\mathcal{B}_{0}} \frac{\Delta(\theta \eta)}{\Delta t} d V-\int_{\mathcal{B}_{0}} \frac{\Delta \theta}{\Delta t} \eta_{\text {algo }} d V-\int_{\mathcal{B}_{0}} R_{\theta n+1 / 2} d V-\int_{\partial_{Q} \mathcal{B}_{0}} Q_{\theta_{n+1 / 2}} d A=0,
\end{aligned}
$$

where use of (51) has been made of on the second term of the left hand side of $(62)_{b}$. Consideration of time independent forces $\boldsymbol{f}_{0}$ and $\boldsymbol{t}_{0}$ and after addition of the three equations in (62), we obtain

$$
\frac{\Delta K}{\Delta t}+\int_{\mathcal{B}_{0}} \frac{1}{\Delta t}\left(\boldsymbol{S}_{\text {algo }}: \frac{1}{2}\left(D \boldsymbol{C}\left[\boldsymbol{w}_{\phi}\right]\right)_{\text {algo }}-\Delta \theta \eta_{\text {algo }}\right) d V+\int_{\mathcal{B}_{0}} \frac{\Delta(\theta \eta)}{\Delta t} d V-\frac{\Delta \prod_{\text {ext }}(\boldsymbol{\phi})}{\Delta t}-\dot{\mathcal{Q}}_{\text {ext }}=0,
$$

From equation (50) and (51) it is possible to re-express the term within the bracket in the second term on the left hand side of $(63)$ as

$$
\boldsymbol{S}_{\text {algo }}: \frac{1}{2}\left(D \boldsymbol{C}\left[\boldsymbol{w}_{\phi}\right]\right)_{\text {algo }}-\Delta \theta \eta_{\text {algo }}=D_{\boldsymbol{C}} \widetilde{W}: \Delta \boldsymbol{C}+D_{\boldsymbol{G}} \widetilde{W}: \Delta \boldsymbol{G}+D_{C} \widetilde{W} \Delta C+D_{\theta} \widetilde{W} \Delta \theta
$$

Introduction of (64) into (63) enables to obtain the following result

$$
\begin{aligned}
& \frac{\Delta K}{\Delta t}+\int_{\mathcal{B}_{0}} \frac{1}{\Delta t}\left(D_{\boldsymbol{C}} \widetilde{W}: \Delta \boldsymbol{C}+D_{\boldsymbol{G}} \widetilde{W}: \Delta \boldsymbol{G}+D_{C} \widetilde{W} \Delta C+D_{\theta} \widetilde{W} \Delta \theta\right) d V \\
& +\int_{\mathcal{B}_{0}} \frac{\Delta(\theta \eta)}{\Delta t} d V-\frac{\Delta \Pi_{\text {ext }}(\phi)}{\Delta t}-\dot{\mathcal{Q}}_{\mathrm{ext}}=0,
\end{aligned}
$$

with

$$
\dot{\mathcal{Q}}_{\text {ext }}=\int_{\mathcal{B}_{0}} R_{\theta n+1 / 2} d V-\int_{\partial_{Q} \mathcal{B}_{0}} Q_{\theta n+1 / 2} d A
$$


When the discrete derivatives $\left\{D_{C} \widetilde{W}, D_{G} \widetilde{W}, D_{C} \widetilde{W}, D_{\theta} \widetilde{W}\right\}$ comply with the directionality property in (52), above equation (65) can be written as

$$
\frac{\Delta K}{\Delta t}+\int_{\mathcal{B}_{0}} \frac{\Delta(\widetilde{W}+\theta \eta)}{\Delta t} d V-\frac{\Delta \Pi_{\text {ext }}(\phi)}{\Delta t}-\dot{\mathcal{Q}}_{\mathrm{ext}}=0
$$

Finally, making use of the Legendre transformation in (28) in (67) enables to obtain the discrete counterpart of the balance of power in (45) as

$$
\frac{\Delta K}{\Delta t}+\int_{\mathcal{B}_{0}} \frac{\Delta \widetilde{U}}{\Delta t} d V-\frac{\Delta \Pi_{\mathrm{ext}}(\phi)}{\Delta t}-\mathcal{Q}_{\mathrm{ext}}=\frac{\Delta \mathcal{H}}{\Delta t}-\frac{\Delta \Pi_{\mathrm{ext}}(\phi)}{\Delta t}-\dot{\mathcal{Q}}_{\mathrm{ext}}=0 .
$$

Therefore, for vanishing external mechanical and thermal power, the Hamiltonian $\mathcal{H}(47)$ is preserved throughout the motion of the continuum in the discrete setting. Three points have been crucial in order to endow the EM time integrator in (48) with the conservation properties shown throughout Section 5.2, namely: (i) the equivalent re-expression (in the continuum level) of the local form of the balance of energy as in equation (6); (ii) the consideration of the algorithmic directional derivatives in equation (50), already introduced in References [17, 27]; (iii) the consideration of the generic definition of the discrete derivatives expressions for $\left\{D_{C} \widetilde{W}, D_{G} \widetilde{W}, D_{C} \widetilde{W}, D_{\theta} \widetilde{W}\right\}$ proposed in Reference [17] and shown in Appendix B for completeness, necessary to guarantee that the discrete derivatives comply with the directionality property in equation (52).

Remark 6. Although not pursued in the example section of this paper, it is possible to define an entropy-based EM time integrator, being a counterpart of that presented in (48). This is presented in Appendix A.

\section{Finite Element implementation}

As standard in finite elements, the domain $\mathcal{B}_{0}$ described in Section 2.1 and representing a thermo-elastic continuum is sub-divided into a finite set of non-overlapping elements $e \in \mathbb{E}$ such that

$$
\mathcal{B}_{0} \approx \mathcal{B}_{0}^{h}=\bigcup_{e \in \mathbb{E}} \mathcal{B}_{0}^{e}
$$

The unknown fields $\{\boldsymbol{v}, \boldsymbol{\phi}, \theta\}$ in the semi-discrete weak forms $\mathcal{W}_{\boldsymbol{v}}, \mathcal{W}_{\boldsymbol{\phi}}$ and $\mathcal{W}_{\theta}$ in (48) are discretised employing the following functional spaces $\mathbb{V}^{h} \times \mathbb{V}^{\phi^{h}} \times \mathbb{V}^{\theta^{h}}$ defined as

$$
\mathbb{V}^{\phi^{h}}=\left\{\boldsymbol{\phi} \in \mathbb{V}^{\phi} ;\left.\quad \phi^{h}\right|_{\mathcal{B}_{0}^{e}}=\sum_{a=1}^{n_{\text {node }}^{\phi}} N_{a}^{\phi} \phi_{a}\right\} ; \quad \mathbb{V}^{\theta^{h}}=\left\{\theta \in \mathbb{V}^{\theta} ;\left.\quad \theta^{h}\right|_{\mathcal{B}_{0}^{e}}=\sum_{a=1}^{n_{\text {node }}^{\theta}} N_{a}^{\theta} \theta_{a}\right\}
$$

where for any field $\mathcal{Y} \in\{\phi, \theta\}, n_{\text {node }}^{\mathcal{Y}}$ denotes the number of nodes per element of the discretisation associated with the field $\mathcal{Y}$ and $N_{a}^{\mathcal{Y}}: \mathcal{B}_{0}^{e} \rightarrow \mathbb{R}$, the $a^{\text {th }}$ shape function used for the interpolation of $\mathcal{Y}$. In addition, $\mathcal{Y}_{a}$ represents the value of the field $\mathcal{Y}$ at the $a^{t h}$ node of a given finite element. Similarly, following a Bubnov-Galerkin approach, the functional spaces for the test functions $\left\{\boldsymbol{w}_{\boldsymbol{v}}, w_{\theta}\right\} \in \mathbb{V}_{0}^{\phi^{h}} \times \mathbb{V}_{0}^{\theta^{h}}$ are defined as

$$
\mathbb{V}_{0}^{\phi^{h}}=\left\{\forall \boldsymbol{\phi} \in \mathbb{V}^{\phi^{h}} ; \quad \boldsymbol{\phi}=\mathbf{0} \text { on } \partial_{\phi} \mathcal{B}_{0}\right\} ; \quad \mathbb{V}_{0}^{\theta^{h}}=\left\{\forall \theta \in \mathbb{V}^{\theta^{h}} ; \quad \theta=0 \text { on } \partial_{\theta} \mathcal{B}_{0}\right\}
$$


Even though the relation between the time derivative of $\boldsymbol{\phi}$ and the velocity field $\boldsymbol{v}$ is imposed in a weak sense (refer to the weak form $\mathcal{W}_{\boldsymbol{v}}$ in $(48)_{a}$ ), the consideration of equal functional spaces for both fields, namely $\phi \in \mathbb{V}^{\phi}$ and $\boldsymbol{v} \in \mathbb{V}^{\phi}$ enables to conclude that (48) $a$ holds strongly, namely

$$
\frac{\Delta \phi}{\Delta t}=\boldsymbol{v}_{n+1 / 2}
$$

Consideration of the functional spaces for $\{\boldsymbol{v}, \boldsymbol{\phi}, \theta\}$ and $\left\{\boldsymbol{w}_{\boldsymbol{v}}, \boldsymbol{w}_{\phi}, w_{\theta}\right\}$ in (70) and (71) enables $\left\{\left(\mathcal{W}_{\boldsymbol{\phi}}\right)_{\text {algo }},\left(\mathcal{W}_{\theta}\right)_{\text {algo }}\right\}$ in $(48)$ to be written in terms of their associated elemental residual contributions, namely

$$
\mathcal{W}_{\boldsymbol{\phi}}=\sum_{e=1}^{N} \boldsymbol{w}_{\phi_{a}} \cdot \boldsymbol{R}_{a, e}^{\phi} ; \quad \mathcal{W}_{\theta}=\sum_{e=1}^{N} w_{\theta a} R_{a, e}^{\theta}
$$

where $N$ denotes the number of elements for the underlying discretisation. The residual contributions $\boldsymbol{R}_{a, e}^{\phi}$ and $R_{a, e}^{\theta}$ can be expressed as ${ }^{7}$

$$
\begin{aligned}
\boldsymbol{R}_{a, e}^{\phi} & =\int_{\mathcal{B}_{0}^{e}} \rho_{0} N_{\phi}^{a}\left(2 \frac{\Delta \boldsymbol{\phi}}{\Delta t^{2}}-2 \frac{\boldsymbol{v}_{n}}{\Delta t}\right) d V+\int_{\mathcal{B}_{0}^{e}}\left(\boldsymbol{F}_{n+1 / 2} \boldsymbol{S}_{\text {algo }}\right) \nabla_{0} N_{a}^{\phi} d V+\int_{\mathcal{B}_{0}^{e}} N_{a}^{\phi} \boldsymbol{f}_{0_{n+1 / 2}} d V ; \\
R_{a, e}^{\theta} & =\int_{\mathcal{B}_{0}} \frac{\Delta(\theta \eta)}{\Delta t} N_{a}^{\theta} d V-\int_{\mathcal{B}_{0}} \frac{\Delta \theta}{\Delta t} \eta_{\text {algo }} N_{a}^{\theta} d V-\int_{\mathcal{B}_{0}} \boldsymbol{Q}_{n+1 / 2} \cdot \nabla_{0} N_{a}^{\theta} d V-\int_{\mathcal{B}_{0}} R_{\theta n+1 / 2} N_{a}^{\theta} d V .
\end{aligned}
$$

where use of equation (72) has been made of in the inertial term of the residual $\boldsymbol{R}_{a, e}^{\phi}$ in $(74)_{a}$. A consistent linearisation of the nonlinear residual contributions (74) has been used in this work.

\section{Numerical examples}

The objective of this section is to study the performance of the newly proposed EM time integration scheme presented in equation (48) in a variety of challenging examples, with the aim of comparing the long-term stability of the new time integrator against that of the classical midpoint rule.

\subsection{Numerical Example 1}

The objectives of this example are:

- O1 To carry out a thorough analysis of the stability and robustness of the proposed EM time integrator comparing it against of the mid-point rule time integrator as a function of the Courant-Friedrich-Lewy number for the case of Finite Element $h$-refinement.

- O2 For a given level of spatial discretisation refinement and time step, to compare the thermodynamical consistency of the proposed time integrator to that of the mid-point time integrator.

The geometry for the problem is displayed in Figure 2. The L-shaped solid is subjected to an external torque induced by a pair of forces $\boldsymbol{F}_{1}(t)$ and $\boldsymbol{F}_{2}(t)$ acting on two of the boundary faces (refer to Figure 2), defined as

$$
\boldsymbol{F}_{2}(t)=-\boldsymbol{F}_{1}(t) ; \quad \boldsymbol{F}_{1}(t)=\left[\begin{array}{c}
256 / 9 \\
512 / 9 \\
768 / 9
\end{array}\right] f(t) ; \quad f(t)=\left\{\begin{array}{rr}
t & 0 \leq t<2.5 \mathrm{~s} \\
5-t & 2.5 \mathrm{~s} \leq t<5 \mathrm{~s} \\
0 & t \geq 5 \mathrm{~s}
\end{array}\right.
$$

\footnotetext{
${ }^{7}$ For simplicity, the external contributions on the boundary of the continuum and associated with $\boldsymbol{t}_{0}$ and $Q_{\theta}$ have not been included in (74).
} 
In addition, initial distribution of temperature on the solid is

$$
\left.\theta(\boldsymbol{X})\right|_{t=0}=\left\{\begin{array}{rr}
T_{1} & Z=L_{Z}, \\
T_{2} & X=L_{X} \\
\theta_{R} & \text { elsewhere. }
\end{array} ; \quad T_{1}=300 \mathrm{~K} ; \quad T_{2}=250 \mathrm{~K} .\right.
$$

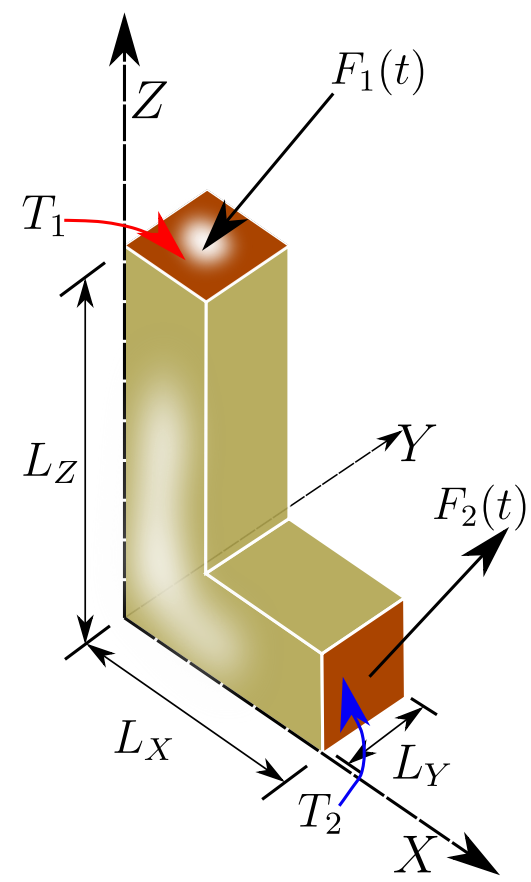

Figure 2: Numerical example 1. Geometry and general setting.

In order to establish a quantitative comparison with the results provided in Reference [17], we use in this example the constitutive model considered therein. This can be expressed in terms of the following additive decomposition,

$$
\widetilde{W}(\boldsymbol{C}, \boldsymbol{G}, C, \theta)=\widetilde{W}_{m}(\boldsymbol{C}, \boldsymbol{G}, C)+\widetilde{W}_{\theta}(\theta)-\left(\theta-\theta_{R}\right) \widetilde{\eta}_{R}(C),
$$

where each of the contributions in (77) is defined as

$$
\begin{aligned}
\widetilde{W}_{m}(\boldsymbol{C}, \boldsymbol{G}, C) & =\frac{\mu_{1}}{2} \operatorname{tr} \boldsymbol{C}+\frac{\mu_{2}}{2} \operatorname{tr} \boldsymbol{G}-\left(\mu_{1}+2 \mu_{2}\right) \ln C^{1 / 2}+\frac{\lambda}{2}\left(C^{1 / 2}-1\right)^{2} ; \\
\widetilde{W}_{\theta}(\theta) & =c_{v}\left(\theta-\theta_{R}-\theta \ln \frac{\theta}{\theta_{R}}\right) ; \quad \widetilde{\eta}_{R}(C)=-3 \beta\left(a\left(C^{1 / 2}-1\right)-b C^{-1 / 2}\right) .
\end{aligned}
$$

The thermal conductivity tensor is particularised for the case of isotropy, whereby it can be expressed in terms of the scalar conductivity field $k$, i.e. $\boldsymbol{k}=k \boldsymbol{I}$. The value of all the relevant material and geometrical parameters in this example can be found in Table 1.

Four different levels of $h$-refinement will be considered in this example. These can be observed in Figure 3. A course, medium, fine and ulta fine finite element meshes (denoted as Mesh1, Mesh2, Mesh3 and Mesh4, respectively) have been considered. With regards to objective $\mathbf{O} 1$, we recall that the Courant-Friedrichs-Lewy number (denoted hereby as $\alpha_{C F L}$ ) is defined as

$$
\alpha_{C F L}=c_{p} \frac{\Delta t}{h} ; \quad c_{p}=\sqrt{\frac{\lambda_{R}+2 \mu_{R}}{\rho_{0}}},
$$

where $\Delta t$ denotes the time step used in the simulations and $h$ the characteristic size of the finite element mesh, and $c_{p}$ the longitudinal wave speed in the reference configuartion $\left(\boldsymbol{\nabla}_{0} \boldsymbol{\phi}=\boldsymbol{I}, \theta=\right.$ 
Table 1: Numerical example 1. Geometrical parameters (see Figure 2) and material parameters (see (78)).

\begin{tabular}{|c|c|c|c|c|}
\hline Geometrical parameters & $\begin{array}{l}L_{X} \\
L_{Y} \\
L_{Z} \\
\end{array}$ & $\begin{array}{c}6 \\
3 \\
10 \\
\end{array}$ & $\begin{array}{l}\mathrm{m} \\
\mathrm{m} \\
\mathrm{m}\end{array}$ & \\
\hline Material parameters & $\begin{array}{c}\mu_{1} \\
\mu_{2} \\
\lambda \\
c_{v} \\
\theta_{R} \\
\beta \\
a \\
b \\
k \\
\rho_{0}\end{array}$ & $\begin{array}{c}1646,7 \\
332,5 \\
0 \\
100 \\
293.15 \\
2,233 \times 10^{-4} \\
\mu_{1}+2 \mu_{2} \\
0 \\
10 \\
100\end{array}$ & $\begin{array}{c}\mathrm{Pa} \\
\mathrm{Pa} \\
\mathrm{Pa} \\
\mathrm{JK}^{-1} \mathrm{~m}^{-3} \\
\mathrm{~K} \\
\mathrm{~K}^{-1} \\
\mathrm{~Pa} \\
\mathrm{~Pa} \\
\mathrm{WK}-1 \mathrm{~m}^{-1} \\
\mathrm{~kg} / \mathrm{m}^{3}\end{array}$ & $\begin{array}{l}\text { (Thermal conductivity) } \\
\text { (Material density) }\end{array}$ \\
\hline
\end{tabular}
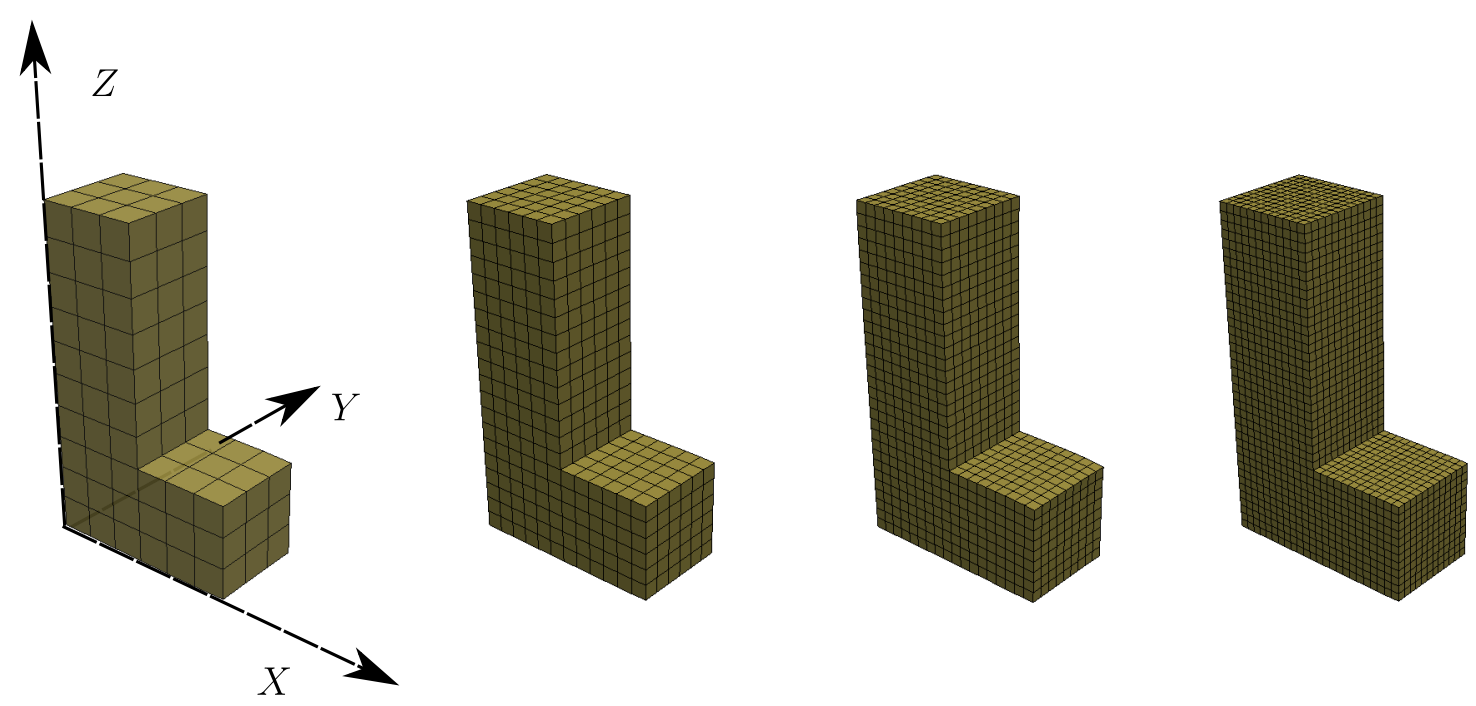

Figure 3: Numerical example 1. $h$-refinement used in the study. $Q_{1}-Q_{1}$ finite element discretisation for both spatial geometry $\phi$ and temperature $\theta$. From left to right: Mesh1 (with $\{672,224\}$ dofs for $\{\phi, \theta\}$ ); Mesh2 (refinement of $\times 2$ in every direction with respect to Mesh1, yielding $\{3822,1274\}$ dofs for $\{\boldsymbol{\phi}, \theta\}$ ); Mesh3 (refinement of $\times 3$ in every direction with respect to Mesh1, yielding $\{12000,4000\}$ dofs for $\{\phi, \theta\}$ ); Mesh4 (refinement of $\times 4$ in every direction with respect to Mesh1, yielding $\{25350,8450\}$ dofs for $\{\phi, \theta\}$ ). 
$\left.\theta_{R}\right)$. For hexahedral meshes, we consider $h$ to be related to the volume of the element $e$ in the mesh (i.e. $V_{e}$ ) and to the order of the Finite Element interpolation $q$ (i.e. $q=1$ for $Q_{1}$ elements, $q=2$ for $Q_{2}$ elements, etc.) as

$$
h=\left(\frac{\min _{e} V_{e}}{q^{3}}\right)^{1 / 3} ; \quad 1 \leq e \leq N,
$$

where $N$ denotes the number of elements for the underlying discretisation. Figure $4_{a}$ shows the final time instant $T_{\text {final }}$ for which the proposed EM time integrator fails, that is, the time step for which the convergence of the iterative Netwon-Raphson algorithm is not achieved [22, 23], for different values of the $\alpha_{C F L}$ number and for the four levels of $h$-refinement displayed in Figure 3. Clearly, for large values of the $\alpha_{C F L}$ number, the new EM time integrator becomes unstable at smaller values of $T_{\text {final }}$, as expected.

Figure $4_{b}$ sheds light with regards to the relative stability of the proposed EM time integrator with respect to that of the classical mid-point time integrator. Specifically, this figure shows the difference between the final time instant for which the proposed EM time integrator and the mid-point rule become unstable. This has been denoted in that figure as $\Delta T_{\text {final }}$. Contrary to the EM time integrator, for the mid-point integrator, the lack of convergence of the Newton-Raphson is always preceded by an uncontrollable growth of the Hamiltonian over the previous time steps. Positive values of $\Delta T_{\text {final }}$ imply that the proposed time integrator becomes unstable at later time instants, and viceversa. It is worth noticing that beyond $\alpha_{C F L} \geq 5-10, \Delta T_{\text {final }}$ adopts negative values. This indicates that the size of the time step $\Delta t$ cannot be chosen arbitrarily large, expecting an improved robustness and stability of the EM time integrator with respect to the classical mid-point rule. In fact our numerical study suggests that $\Delta t$ must be chosen such that $\alpha_{C F L} \leq 5$ in order to guarantee a higher stability and robustness in the long term.
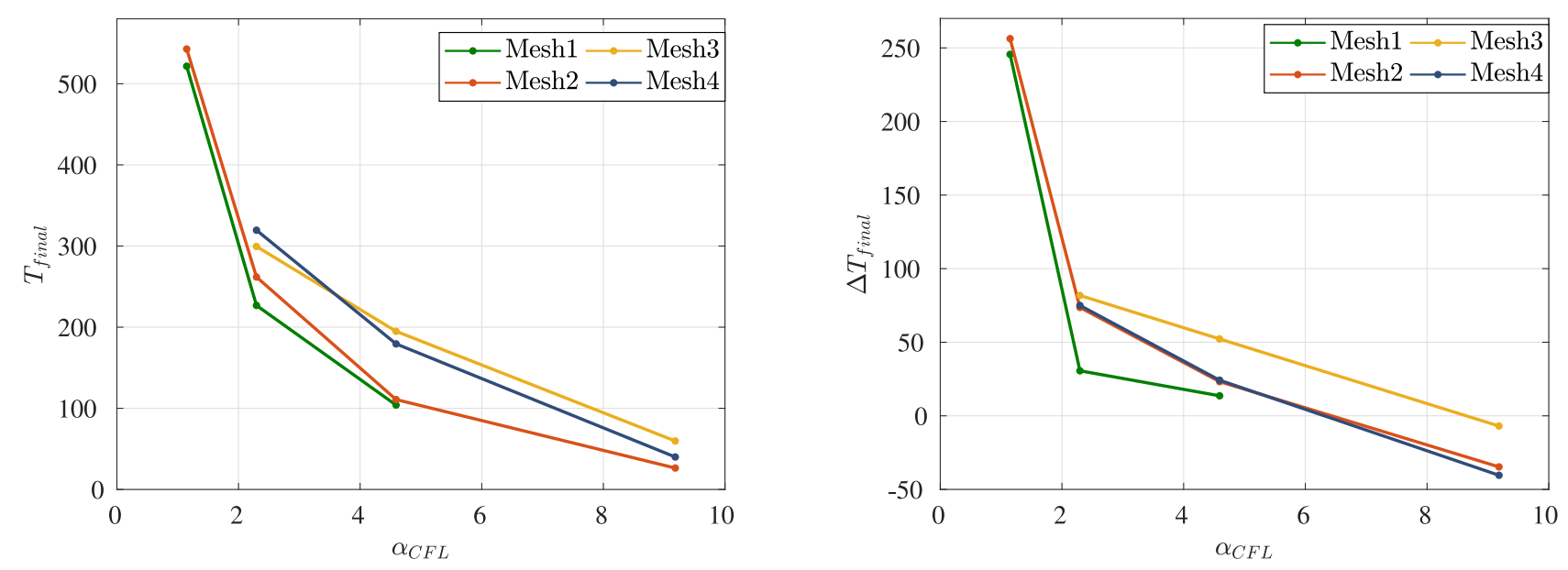

Figure 4: Numerical example 1. Left: final time instant $\left(T_{\text {final }}\right)$ for which the EM time integrator becomes unstable. Rigth: Difference between the final time instant $\left(\Delta T_{\text {final }}\right)$ at which the EM time integrator and the mid-point rule become unstable. Results shown for various values of $\alpha_{C F L}$ and for the discretisations in Figure 3 .

With regards to objective $\mathbf{0 2}$, we use in this study the computational domain defined by Mesh2 (see Figure 3) and a value of $\alpha_{C F L}$ of $\alpha_{C F L}=2.2955$. It can be seen that for both the new EM time integrator and the mid-point rule the angular momentum $\boldsymbol{J}$ remains constant beyond $t \geq 5 \mathrm{~s}$, when the external applied pair of forces vanishes. In addition, the linear momentum $\boldsymbol{L}$ is zero for the entire simulation. Another interesting variable of interest is the global entropy $\left(\tilde{\eta}=\int_{\mathcal{B}_{0}} \eta d V\right)$, which increases over time for the entire simulation for both time integrators. Furthermore, the Hamiltonian $\mathcal{H}$ is displayed. The zoomed detail perfectly shows the sudden 

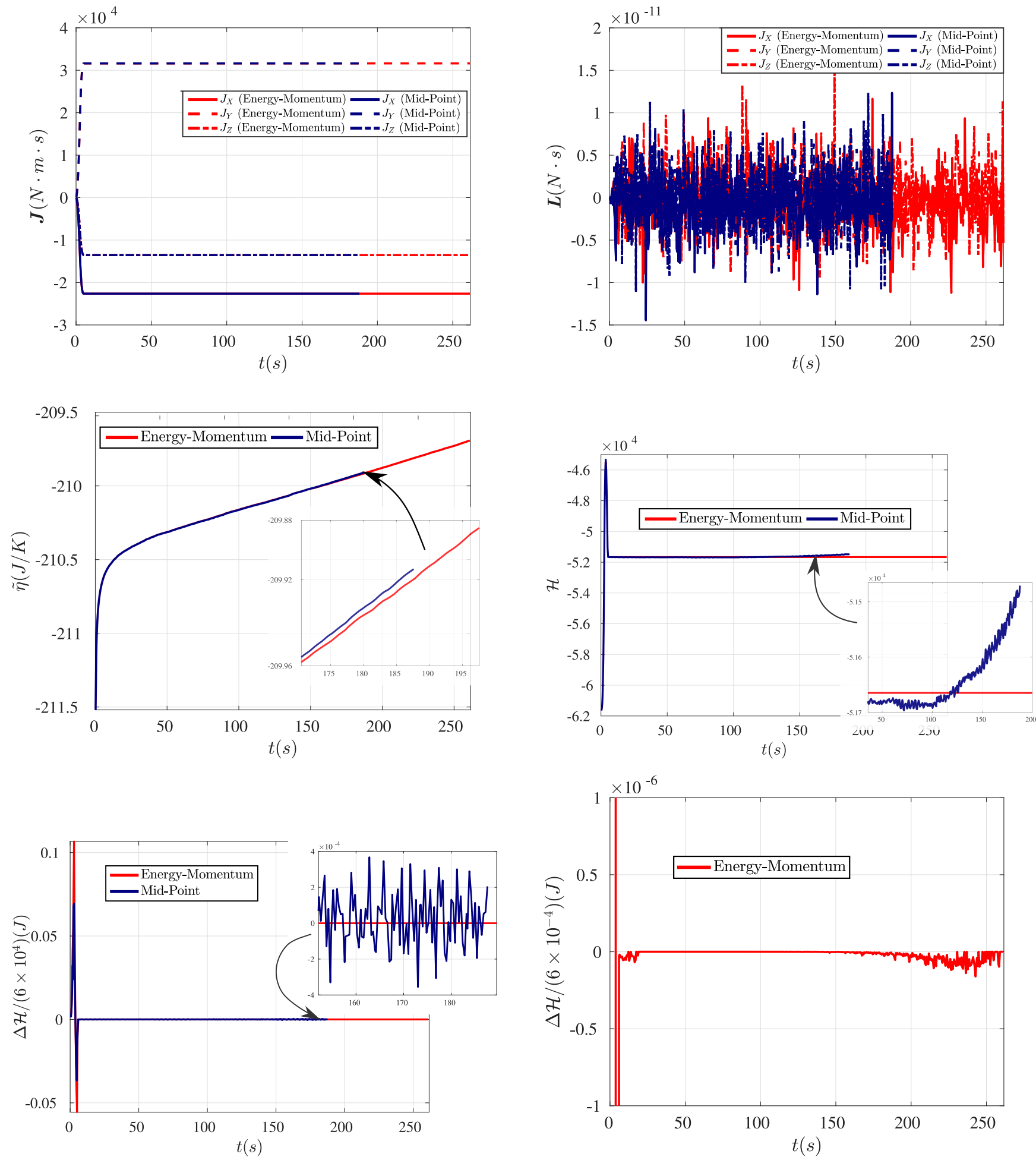

Figure 5: Numerical example 1. Evolution of: (a) angular momentum $\boldsymbol{J}$, (b) linear momentum $\boldsymbol{L}$, (c) global entropy $\tilde{\eta}=\int_{\mathcal{B}_{0}} \eta d V$, (d) Hamiltonian $\mathcal{H}$ in (47), (e) increment of Hamiltonian $\Delta \mathcal{H}$ for both the Mid-Point and the new EM time integrator. Finally, (f), zoomed detail of the increment of the Hamiltonian $\Delta \mathcal{H}$ for the new EM time integrator. Results obtained for Mesh2 with $\alpha_{C F L}=2.2955(\Delta t=0.2 \mathrm{~s})$. 
increase in the Hamiltonian $\mathcal{H}$ prior to the instability when using the mid-point rule. The increase of the Hamiltonian $\Delta \mathcal{H}=\mathcal{H}_{n+1}-\mathcal{H}_{n}$ (normalised with respect to the maximum historic value of $\mathcal{H}$ in absolute value) is also displayed. It can be seen that the new EM time integrator preserves $\mathcal{H}$ (beyond $t \geq 5 \mathrm{~s}$ ) as it has been designed specifically with that purpose, whereas the mid-point rule does not.

Finally, the temperature contour plot is displayed over time in Figure 6. The results have been obtained by means of the new EM time integrator using a different mesh from the four depicted in Figure 3. This mesh has been generated refining by a factor of $\times 6$ in the three directions the computational domain defined by Mesh1.

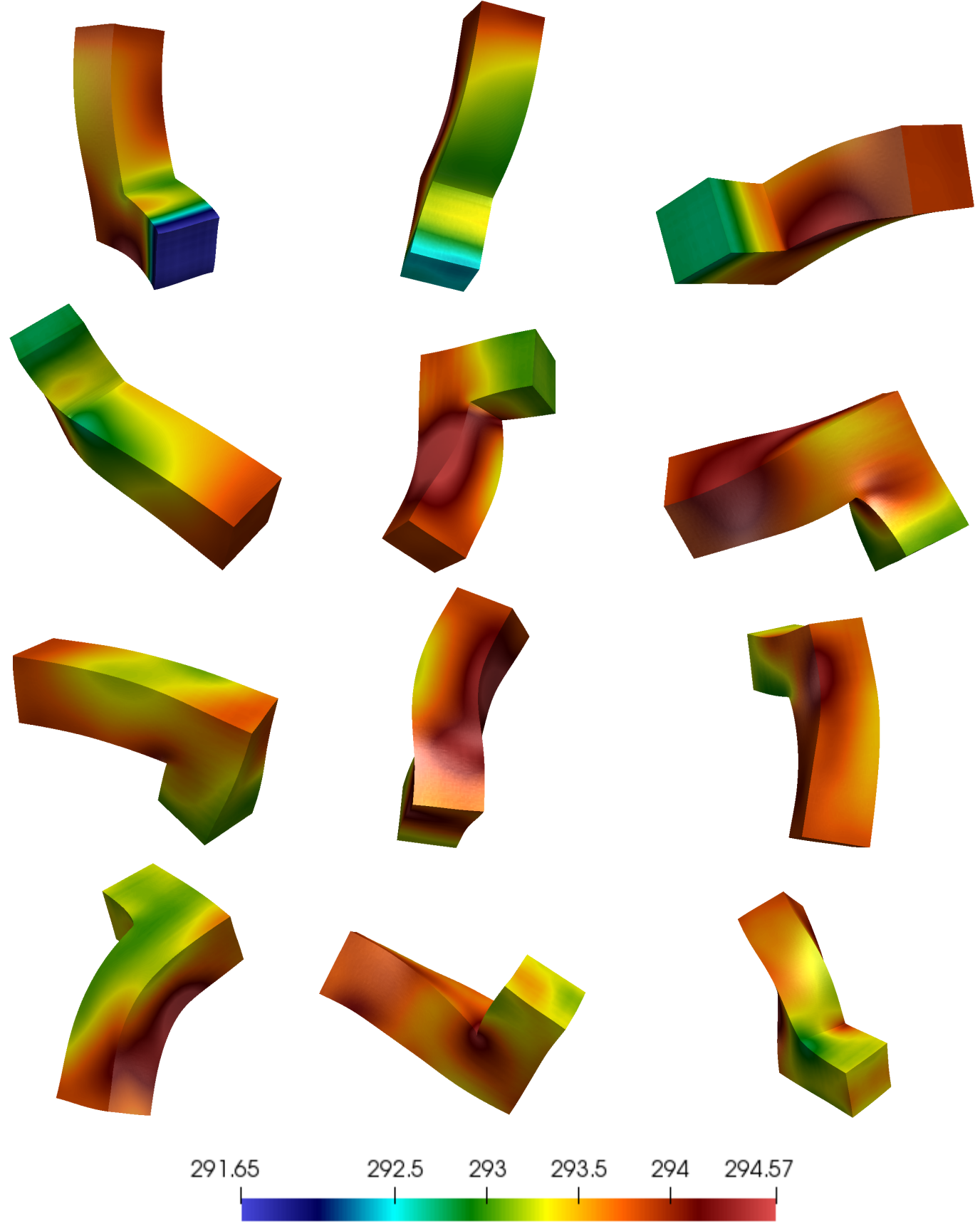

Figure 6: Numerical example 1. Contour plot distribution of absolute temperature $T(K)$ for $t=$ $\{2.5,5,8,11,15,19,21,25,33,35,40,43\} \mathrm{s}$ (from left to right and top to bottom). $Q_{1}-Q_{1}$ discretisation for both spatial geometry $\boldsymbol{\phi}$ and temperature $\theta$. Number of dofs in the mesh: $\{85557,28519\}$ for $\{\boldsymbol{\phi}, \theta\}$. Results obtained by means of the new EM time integrator for $\alpha_{C F L}=3.44(\Delta t=0.1 \mathrm{~s})$. 


\subsection{Numerical Example 2}

The objective of this example is

- O1 Study the stability and robustness in a problem where the deformation is exclusively induced by thermal effects.

The geometry for this example is displayed in Figure 7. The object in Figure 7 is subjected to a heat flux at the bottom surface (minimum coordinate $Z$ ), characterised by the following mathematical expression

$$
Q_{\theta}(t)=Q_{\max } \sin \frac{2 \pi}{T} t ; \quad T=1 \mathrm{~s} ; \quad Q_{\max }=3000 \mathrm{~W} / \mathrm{m}^{2} .
$$
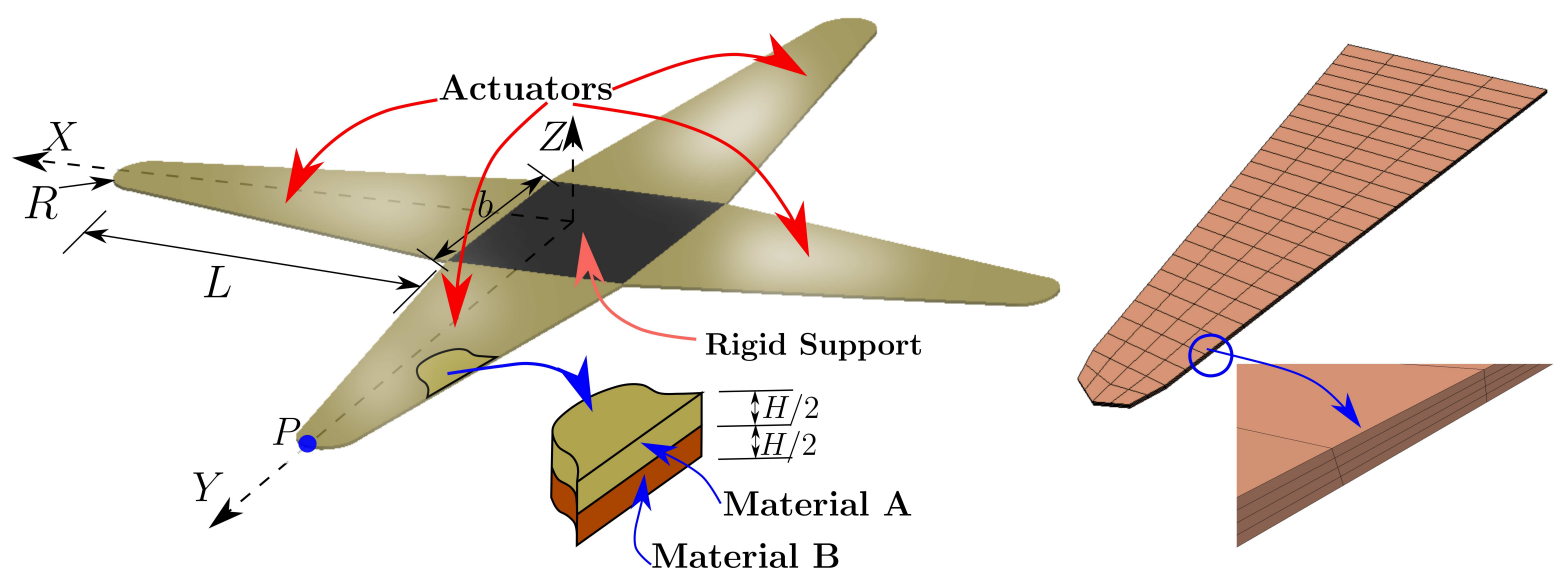

Figure 7: Numerical example 2. Left: geometry and general setting for the bi-material thermo-mechanical actuator. Right: computational domain considered for the analysis of $\mathbf{O} \mathbf{1}$, based on a $Q_{2}$ discretisation for $\{\boldsymbol{x}, T\}$, $(\{1215,405\}$ dofs $)$. Every $Q_{2}$ finite element has been divided into $2 \times 2 \times 2$ elements for visualisation purposes.

The constitutive model used in this example is that presented in Section 3.1.1 through equations (18), (26), (21) and (22). The value of the material parameters in that model can be found in Table 2.

With regards to objective $\mathbf{O 1}$, we use in this study the computational domain defined in Figure 7 and several values of $\alpha_{C F L}$. From Figure $8_{(a)^{-}(b)}$ it can be observed that the range of stability of the new EM time integrator is larger than that of the mid-point rule for approximately $\alpha_{C F L} \leq 10$ (close to zero and even negative values of $\Delta T_{\text {final }}$ are obtained in the range $\alpha_{C F L} \geq 10$ ). For a fixed value of $\alpha_{C F L}=5.6\left(\Delta t=4 \times 10^{-4} \mathrm{~s}\right)$, Figure $8_{(c)}$ shows the evolution of the Hamiltonian in equation (47) for both the new EM time integrator and the mid-point rule. In this case, the non-vanishing heat flux $Q_{\theta}$ (81) prevents the Hamiltonian from being preserved. Nonetheless, from equations (46), (47) and (68), it is clear that the quantity of interest defined as

$$
\widetilde{\mathcal{H}}=\mathcal{H}-\Pi_{\text {ext }}-\mathcal{Q}_{\text {ext }}
$$

must be preserved by the new EM time integrator. This in fact confirmed in Figure $8_{(d)}$, where $\widetilde{\mathcal{H}}$ is perfectly preserved throughout the entire simulation by the new proposed time integrator. On the contrary, $\widetilde{\mathcal{H}}$ increases over time when using the mid-point time integrator until it finally becomes unstable. Finally, Figure 10 shows the pressure contour plot over various snapshots computed by means of the new EM time integrator using the computational domain defined in Figure 9. 
Table 2: Numerical example 2. Geometrical parameters (see Figure 7) and material parameters (see (78)).

\begin{tabular}{|c|c|c|c|c|}
\hline Geometrical parameters & $\begin{array}{c}L \\
b \\
H \\
R\end{array}$ & $\begin{array}{c}0,12 \\
0,06 \\
0,001 \\
0,01\end{array}$ & $\begin{array}{l}\mathrm{m} \\
\mathrm{m} \\
\mathrm{m} \\
\mathrm{m}\end{array}$ & \\
\hline Material parameters A & $\begin{array}{l}\mu_{1} \\
\mu_{2}^{A} \\
\lambda^{A} \\
c_{v}^{A} \\
\theta_{R}^{A} \\
\Gamma_{0}^{A} \\
q^{A} \\
k^{A} \\
\rho_{0}^{A}\end{array}$ & $\begin{array}{c}41.67 \\
0 \\
27.78 \\
2000 \\
293,15 \\
0 \\
1 \\
10 \\
1000\end{array}$ & $\begin{array}{c}\mathrm{kPa} \\
\mathrm{Pa} \\
\mathrm{Pa} \\
\mathrm{JK}^{-1} \mathrm{~m}^{-3} \\
\mathrm{~K} \\
\\
\mathrm{WK}^{-1} \mathrm{~m}^{-1} \\
\mathrm{~kg} / \mathrm{m}^{3}\end{array}$ & $\begin{array}{l}\text { (Specific heat capacity) } \\
\text { (Reference temperature) } \\
\text { (Thermal conductivity) } \\
\text { (Material density) }\end{array}$ \\
\hline Material parameters B & $\begin{array}{l}\mu_{1}^{B} \\
\mu_{2}^{B} \\
\lambda^{B} \\
c_{v}^{B} \\
\theta_{R}^{B} \\
\Gamma_{0}^{B} \\
q^{B} \\
k^{B} \\
\rho_{0}^{B}\end{array}$ & $\begin{array}{c}250 \\
0 \\
166 \\
2000 \\
293,15 \\
0.01 \\
1 \\
10 \\
1000\end{array}$ & $\begin{array}{c}\mathrm{kPa} \\
\mathrm{Pa} \\
\mathrm{Pa} \\
\mathrm{JK}^{-1} \mathrm{~m}^{-3} \\
\mathrm{~K} \\
\\
\mathrm{WK}^{-1} \mathrm{~m}^{-1} \\
\mathrm{~kg} / \mathrm{m}^{3}\end{array}$ & $\begin{array}{l}\text { (Specific heat capacity) } \\
\text { (Reference temperature) } \\
\text { (Thermal conductivity) } \\
\text { (Material density) }\end{array}$ \\
\hline
\end{tabular}




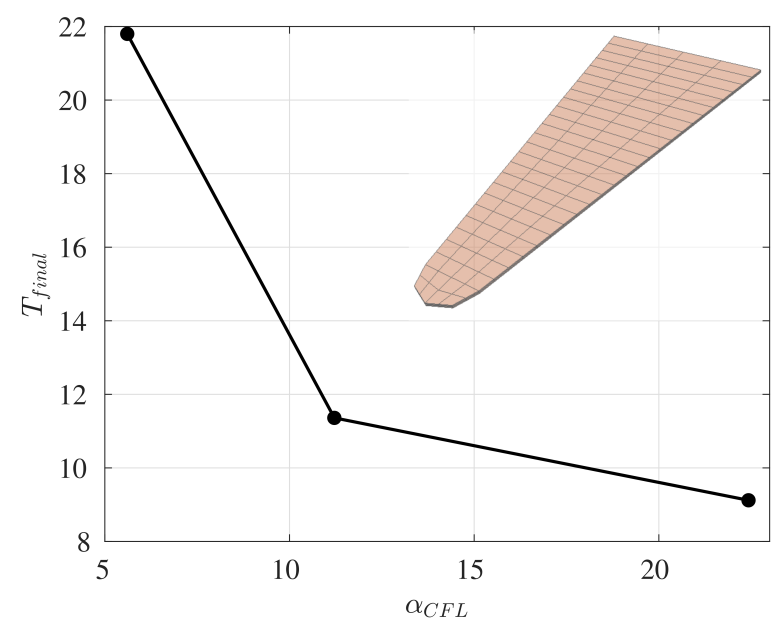

(a)

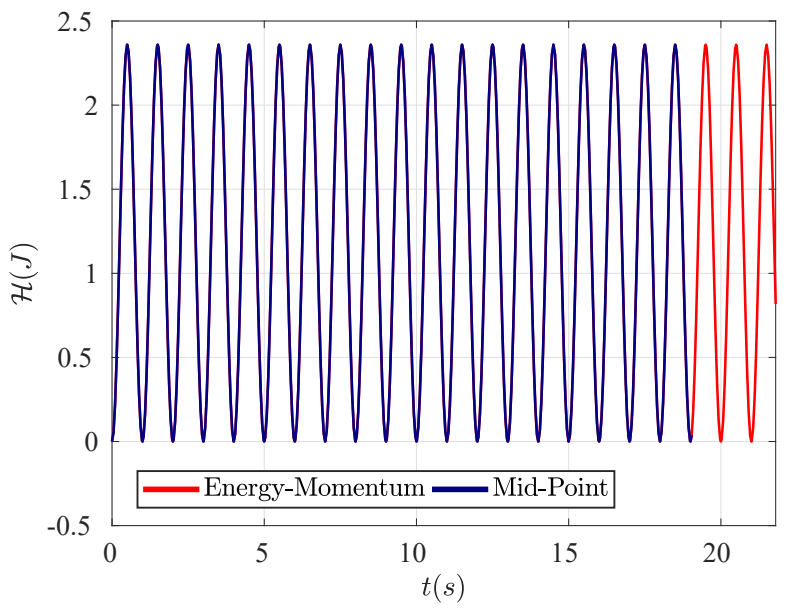

(c)

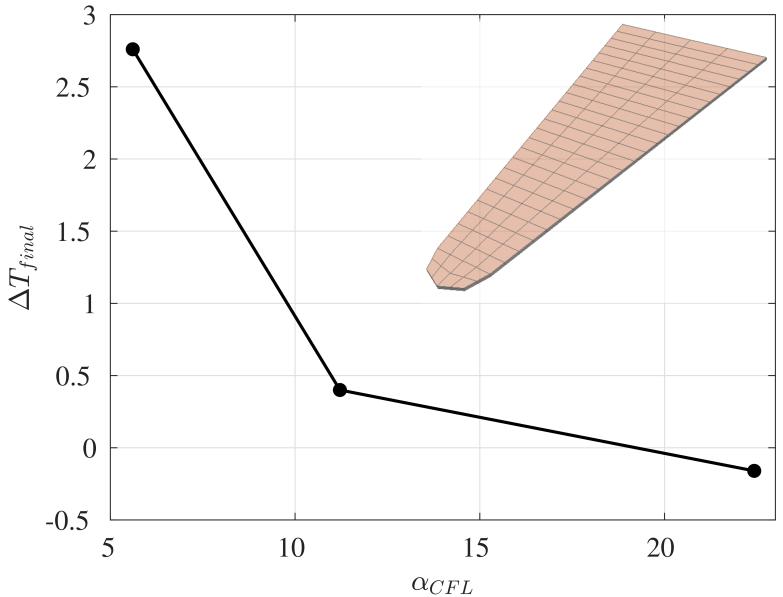

(b)

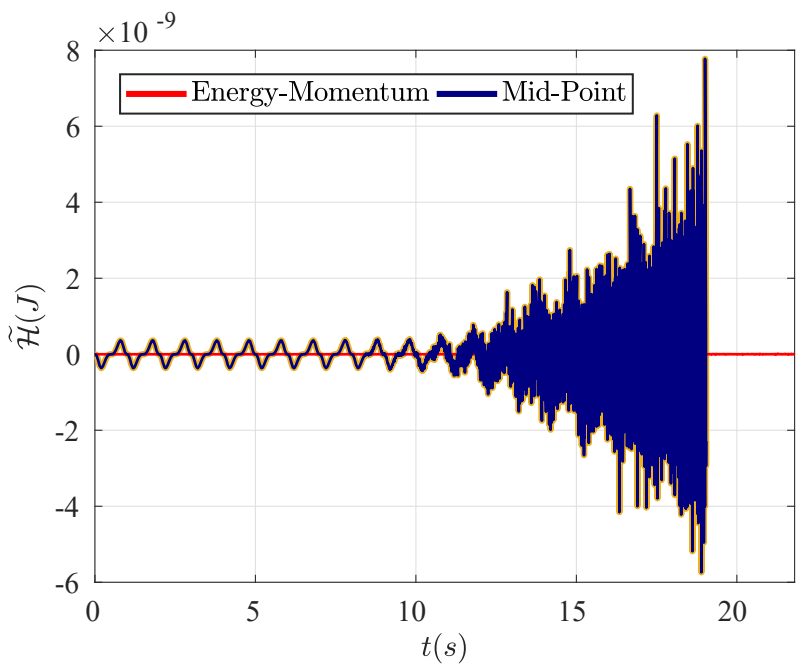

(d)

Figure 8: Numerical example 2. (a) Final time instant $\left(T_{\text {final }}\right)$ for which the EM time integrator becomes unstable. (b) Difference between the final time instant $\left(\Delta T_{\text {final }}\right)$ at which the EM time integrator and the mid-point rule become unstable. Results shown for various values of $\alpha_{C F L}=\{5.6,11.21,22.42\}\left(\Delta t=\{4,8,16\} \times 10^{-4} \mathrm{~s}\right)$ and for the computational domain displayed. (c) Evolution of the Hamiltonian $\mathcal{H}(47)$ when using the mid-point rule and the new EM time integrator for $\alpha_{C F L}=5.6$. (d) Evolution of the quantity of interest $\widetilde{\mathcal{H}}$ in (82) for both mid-point rule and the new EM time integrator for $\alpha_{C F L}=5.6$.

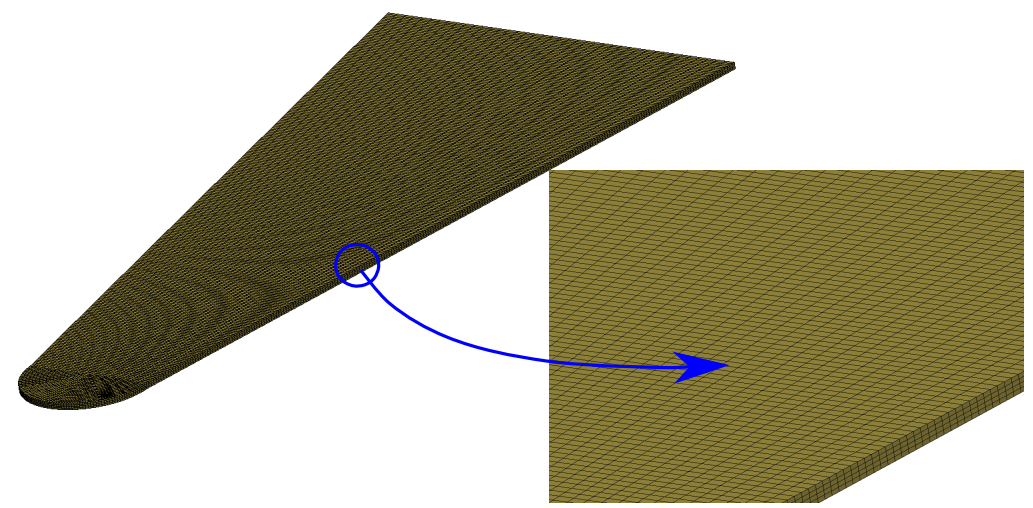

Figure 9: Numerical example 2. Computational domain discretised with $Q_{2}$ elements for both geometry $\phi$ and temperature $\theta$. Discretisation of $\{164430,54810\}$ dofs for $\{\boldsymbol{\phi}, \theta\}$. In the figure every $Q_{2}$ finite element has been divided into $2 \times 2 \times 2$ elements for visualisation purposes. 

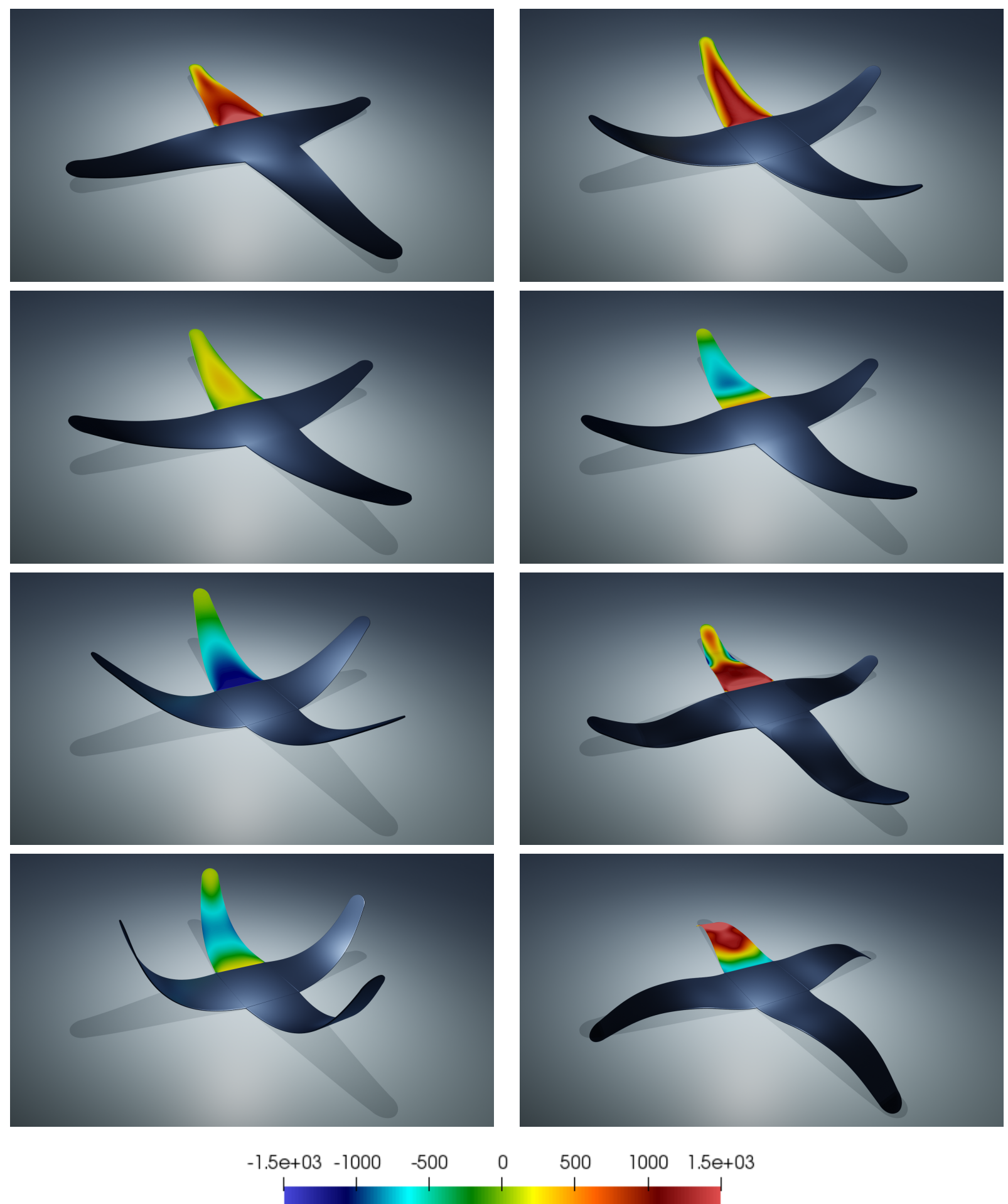

Figure 10: Numerical example 2. Rendering of deformed configuration and contour plot distribution of hydrostatic pressure $p$ for snapshots corresponding to: $t=\{0.41,1.01,1.81,2.61,3.41,3.81,4.41,5.01\} \mathrm{s}$ (from top to bottom and left to right). Results obtained with new EM time integrator and with $\alpha_{C F L}=6.4\left(\Delta t=10^{-4} \mathrm{~s}\right)$. Computational domain in Figure 9. 


\subsection{Numerical Example 3}

The objective of this example is

- O1 Confirmation of the results provided in the previous examples, in terms of stability and robustness, in a challenging numerical example.

The geometry for this example is displayed in Figure 11. The squared object in Figure 11 is subjected to an initial velocity profile given by the following equation

$$
\left.\boldsymbol{v}\right|_{t=0}=\sqrt{\frac{2}{\pi}}\left(\exp \left(-\frac{(X-5)^{2}}{10}\right)+\exp \left(-\frac{(Y-5)^{2}}{10}\right)\right)\left[\begin{array}{l}
0 \\
0 \\
1
\end{array}\right](\mathrm{m} / \mathrm{s}) .
$$

In addition, the object in Figure (11) is initially subjected to a uniform temperature distribution of $\left.\theta\right|_{t=0}=\theta_{R}$, and a heat flux $Q_{\theta}$ defined as

$$
Q_{\theta}(t)=\left\{\begin{array}{rr}
\frac{10^{4}}{4 \pi R^{2}}\left(\mathrm{~W} / \mathrm{m}^{2}\right) & 0 \leq t<2 \mathrm{~s} \\
0 & t \geq 2 \mathrm{~s}
\end{array}\right.
$$
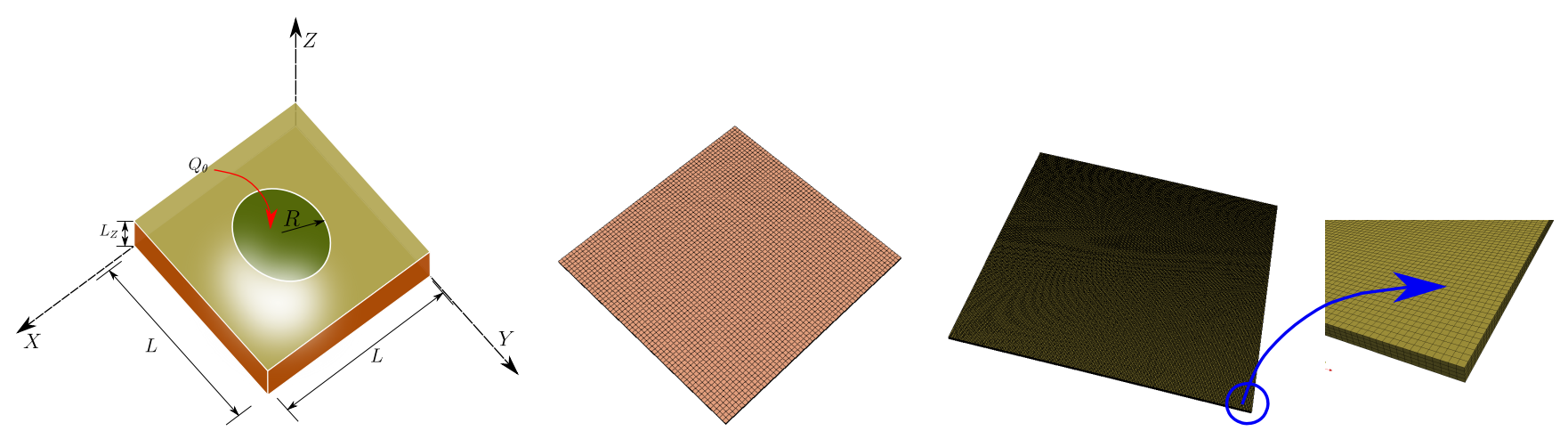

Figure 11: Numerical example 3. Left: geometry with $\left\{L, L_{Z}, R\right\}=\{10,0.1,1.5\}(m)$. Centre: computational domain considered for the analysis of $\mathbf{O 1}$, based on a $Q_{2}$ discretisation for $\{\boldsymbol{\phi}, \theta\}$. Symmetric boundary conditions have been applied, hence only a quarter of the domain displayed has been simulated, yielding $\{1215,405\}$ dofs. Right: Computational domain considered for simulations in Figures 13-16, discretised with $Q_{2}$ elements for both geometry $\phi$ and temperature $\theta$. Symmetric boundary conditions have been applied, hence only a quarter of the domain displayed has been simulated, yielding $\{219615,73205\}$ dofs for $\{\phi, \theta\}$. In the figure every $Q_{2}$ finite element has been divided into $2 \times 2 \times 2$ elements for visualisation purposes.

The constitutive model used in this example is that presented in Section 3.1.1 through equations (18), (26), (21) and (22). The value of the material parameters in that model can be found in Table 3.

With regards to objective $\mathbf{O 1}$, we use in this study the computational domain defined in Figure $11_{b}$ and a value of $\alpha_{C F L}$ of $\alpha_{C F L}=1.72(\Delta t=0.02 \mathrm{~s})$. From Figure 12, it can be seen the variation of angular momentum $\Delta \boldsymbol{J}$, linear momentum $\Delta \boldsymbol{L}$, global entropy $(\tilde{\eta})$ and Hamiltonian $\mathcal{H}$, for both the new EM time integrator and the mid-point rule. Furthermore, the Hamiltonian $\mathcal{H}$ is displayed. The zoomed detail perfectly shows the sudden increase in the Hamiltonian $\mathcal{H}$ prior to the instability when using the mid-point rule. The increase of the Hamiltonian $\Delta \mathcal{H}=\mathcal{H}_{n+1}-\mathcal{H}_{n}$ is also displayed. It can be seen that the new EM time integrator preserves $\mathcal{H}$ (beyond $t \geq 2 \mathrm{~s}$ ), whereas the mid-point rule does not. It is interesting to observe that since only a quarter of the domain has been simulated, the introduction of symmetric boundary conditions introduces a reaction force which prevents the global linear and angular momentum $(\boldsymbol{L}$ and $\boldsymbol{J})$ to be preserved. Only the $Z$ component of $\boldsymbol{L}$ and $\boldsymbol{J}$ is preserved throughout the 

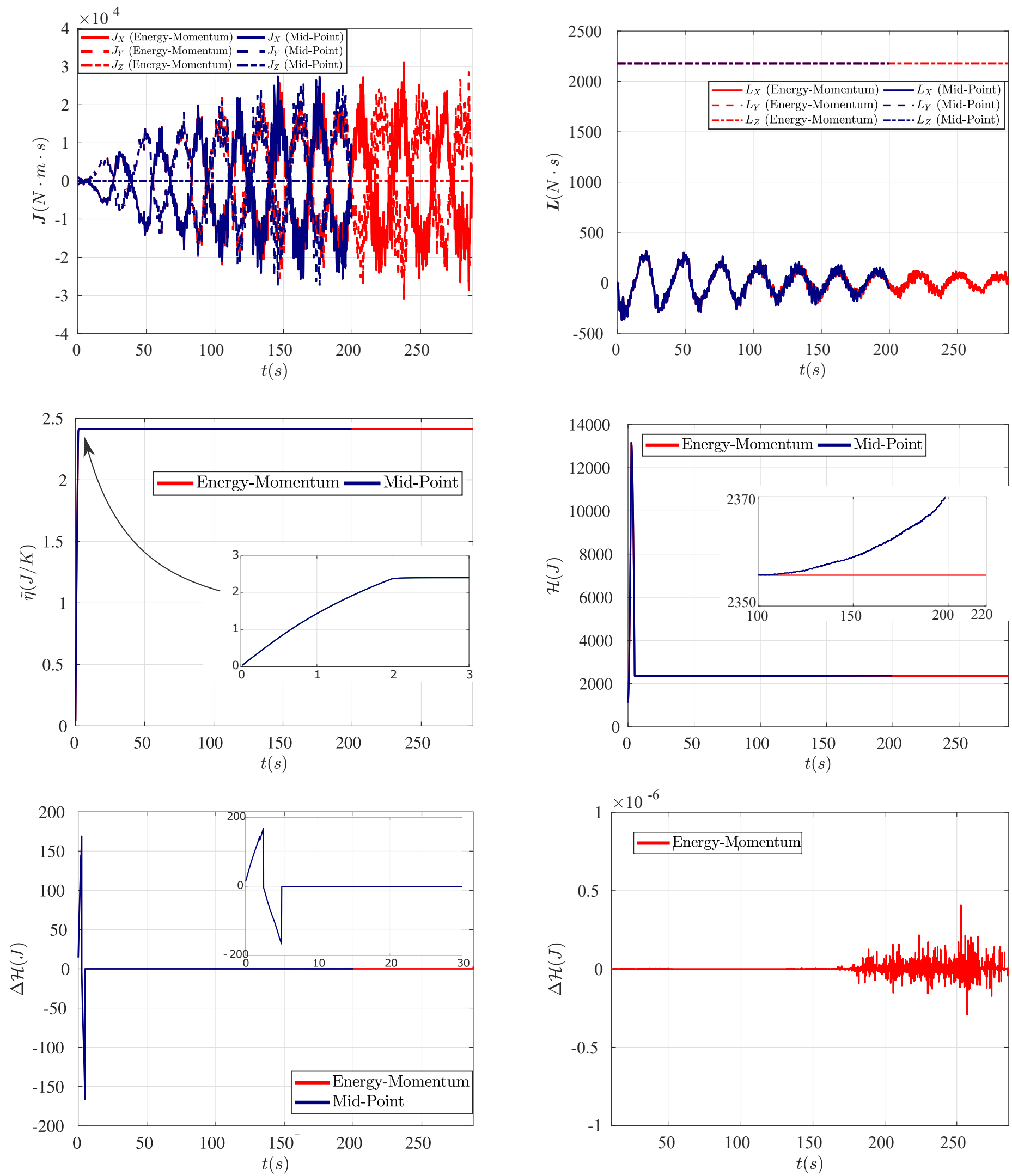

Figure 12: Numerical example 3. Evolution of: (a) angular momentum $\boldsymbol{J}$, (b) linear momentum $\boldsymbol{L}$, (c) global entropy $\tilde{\eta}=\int_{\mathcal{B}_{0}} \eta d V$, (d) Hamiltonian $\mathcal{H}$ in (47), (e) increment of Hamiltonian $\Delta \mathcal{H}$ for both the Mid-Point and the new EM time integrator. Finally, (f), zoomed detail of the increment of the Hamiltonian $\Delta \mathcal{H}$ for the new EM time integrator. Results obtained for mesh in Figure 11 with $\alpha_{C F L}=1.72(\Delta t=0.02 \mathrm{~s})$. 
Table 3: Numerical example 3. Geometrical parameters (see Figure 11) and material parameters (see (78)).

\begin{tabular}{l|cccc|} 
Geometrical parameters & \multicolumn{5}{c}{} & \\
& $L$ & 10 & $\mathrm{~m}$ & \\
& $L_{Z}$ & 0.1 & $\mathrm{~m}$ & \\
$R$ & 1.5 & $\mathrm{~m}$ & \\
\hline \multirow{5}{*}{ Material parameters } & & & $\mathrm{kPa}$ & \\
& $\mu_{1}$ & 19,42 & $\mathrm{~Pa}$ & \\
& $\mu_{2}$ & 0 & $\mathrm{~Pa}$ & \\
$\lambda$ & 29,13 & $\mathrm{JK}^{-1} \mathrm{~m}^{-3}$ & (Specific heat capacity) \\
& $c_{v}$ & 1 & & (Reference temperature) \\
$\theta_{R}$ & 308,15 & $\mathrm{~K}$ & \\
$\Gamma_{0}$ & $6,7 \times 10^{-4}$ & & & \\
$q$ & 1 & & & \\
$k$ & 10 & $\mathrm{WK}^{-1} \mathrm{~m}^{-1}$ & (Thermal conductivity) \\
& $\rho_{0}$ & 1000 & $\mathrm{~kg} / \mathrm{m}^{3}$ & (Material density)
\end{tabular}

simulation (for both time integrators), as the symmetric boundary conditions only affect the $X$ and $Y$ directions.

Finally, we consider the computational domain defined in Figure $11_{c}$. Figure 13 displays the pressure contour plot distribution for various time snapshots. Furthermore, Figure 14 shows the wrinkling pattern that forms over the surface of the plate over time. The wrinkles can be better appreciated in Figure 15. Finally, Figure 16 shows the evolution of the $Z$ components of the displacement of the centroid of the plate over time, induced by the initial velocity profile in (83). 

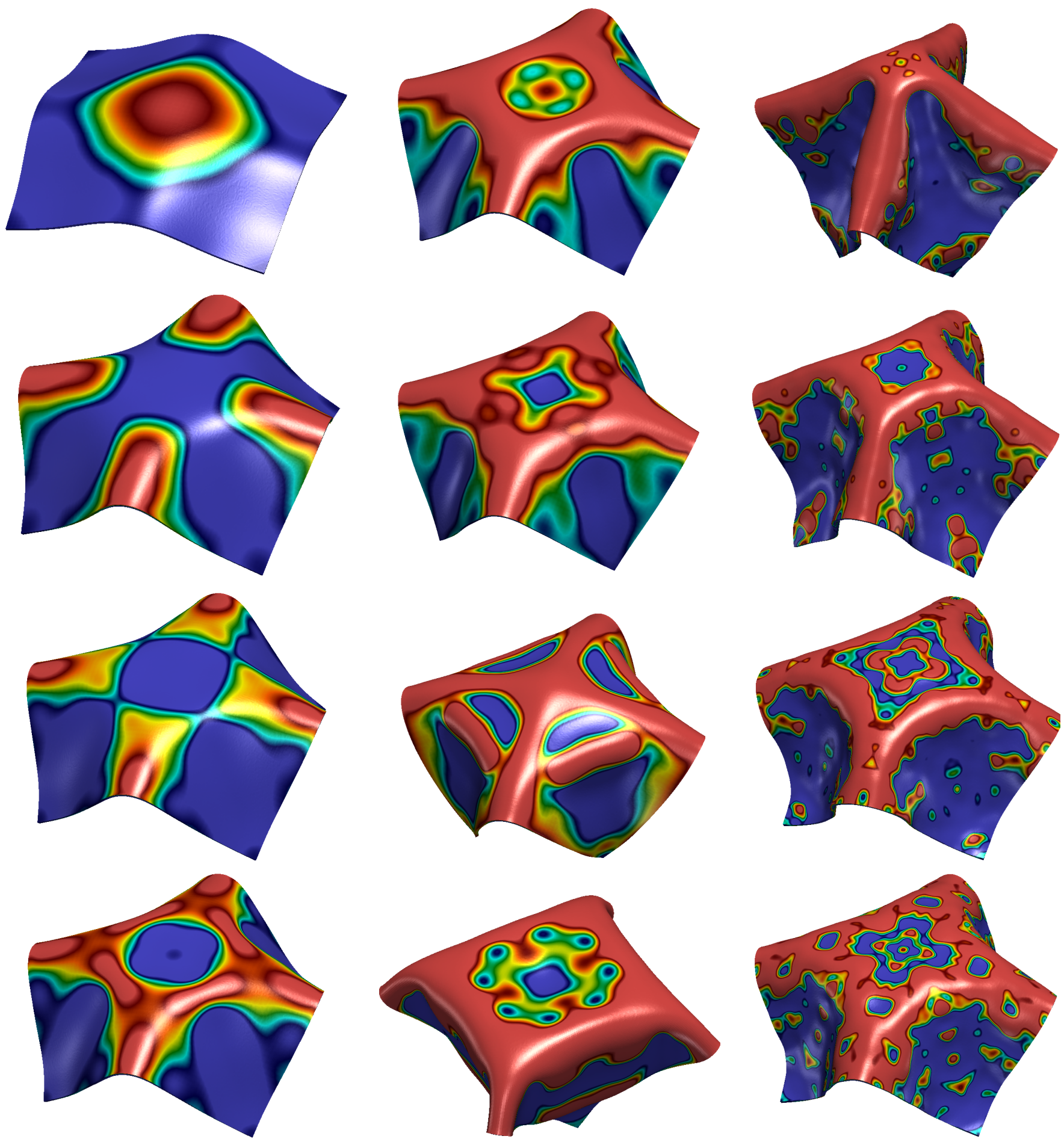

$-3.5 e+020$

500

100

$1500 \quad 2000 \quad 2500$

$3.2 \mathrm{e}+03$

Figure 13: Numerical example 3. Contour plot of hydrostatic pressure $p=\frac{1}{3 \mathrm{~J}} \boldsymbol{S}: \boldsymbol{C}$ at time steps $t=$ $\{1.98,3.58,3.98,4.18,4.38,4.98,5.98,9.36,23.62,26.60,28.52,29.28\} \mathrm{s}$ (from left to right and top to bottom). Results of obtained by means of the new EM time integrator for $\alpha_{C F L}=4.7(\Delta t=0.02 \mathrm{~s})$. Computational domain in Figure $11_{c}$. The results do not show the vertical ( $Z$ direction) elevation of the plate (refer to Figure 16). 


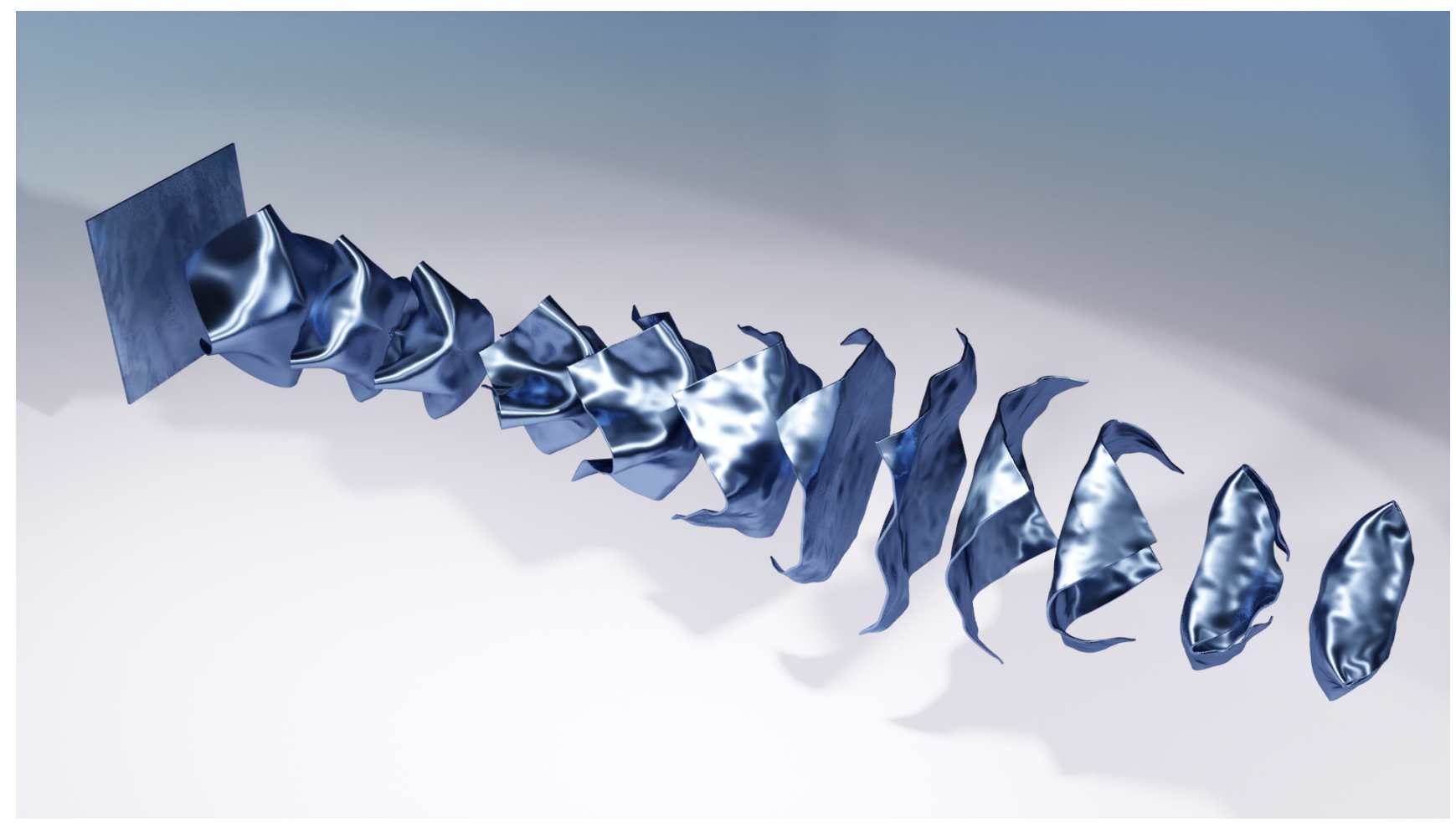

Figure 14: Numerical example 3. Rendering of results for deformed configuration at various time steps (from top to bottom and from left to right). Results obtained by means of the new EM time integrator for $\alpha_{C F L}=4.7(\Delta t=$ $0.02 \mathrm{~s})$. Computational domain in Figure $11_{c}$.

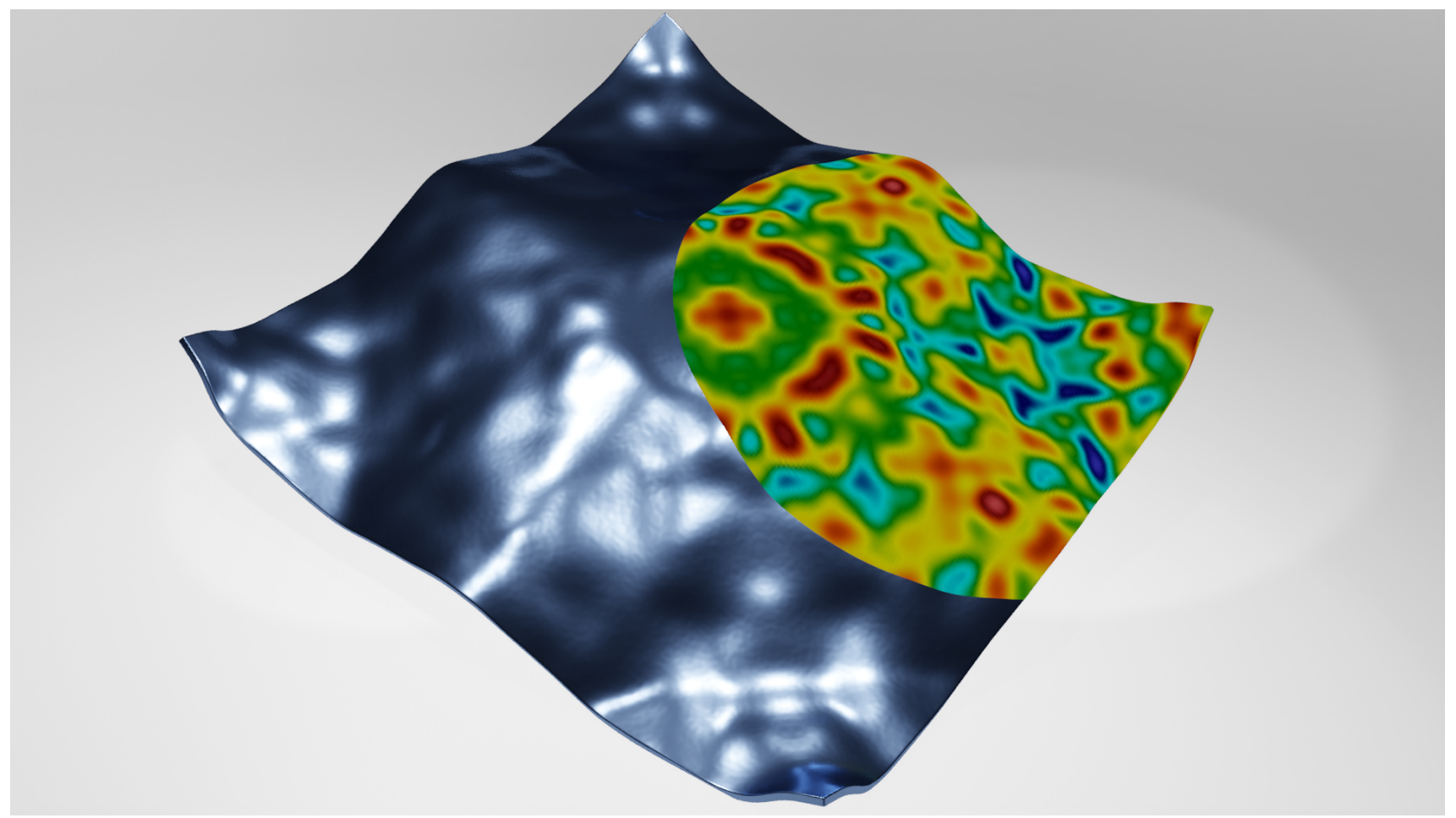

Figure 15: Numerical example 3. Rendering of wrinkling pattern over the surface of the thin plate at time $t=23.62 \mathrm{~s}$. Results obtained with the new EM time integrator for $\alpha_{C F L}=4.7(\Delta t=0.02 \mathrm{~s})$. Computational domain in Figure $11_{c}$. 


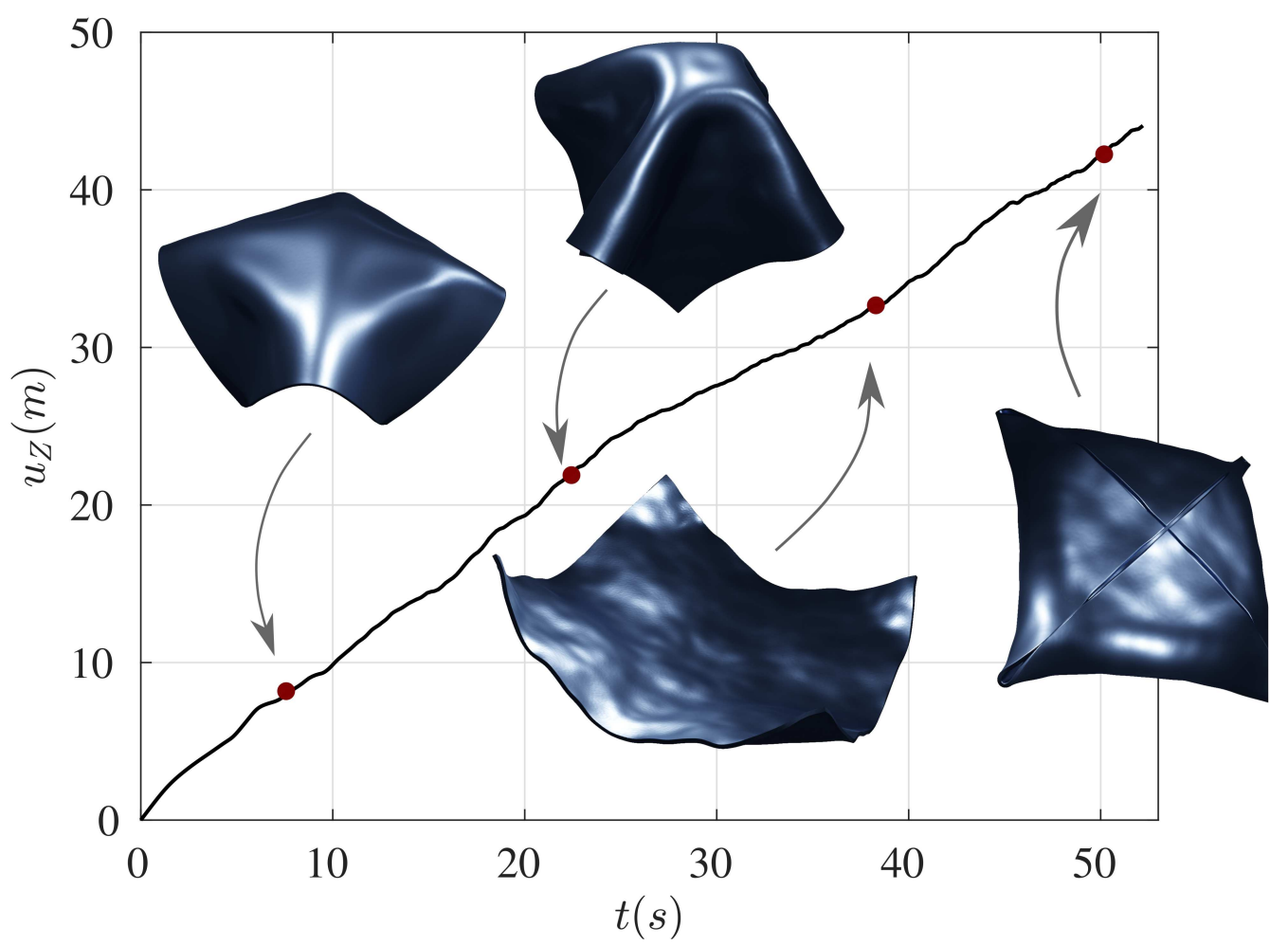

Figure 16: Numerical example 3. Evolution of the displacement in $Z$ direction of the centroid of the thin plate in Figure 11 with respect to time $(t)$. Deformed configuration at selected snapshots. 


\section{Conclusions}

A new one-step implicit and thermodynamically consistent Energy-Momentum (EM) preserving time integration scheme has been presented for the simulation of structural components undergoing large deformations and temperature fields, for which well-posed constitutive models are used for the entire range of deformations and temperature. The use of polyconvexity inspired constitutive models and the new tensor cross product algebra pioneered by de Boer [15] and re-discovered by Bonet et al. [10] in the context of nonlinear solid mechanics, are key for the development of the discrete derivatives, fundamental for the construction of the EM algorithmic derived variables, namely the second Piola-Kirchoff stress tensor and the entropy (or the absolute temperature). The proposed scheme inherits the advantages of the EM scheme recently published by Franke et al. [17] (i.e. consistency, stability, conservation) whilst resulting in dramatically far simpler algorithmic expressions, thus circumventing a bottleneck and paving the way for the incorporation of further physics into the model. A series of numerical examples have been presented in order to demonstrate the robustness and applicability of the new EM scheme. These examples make use of a temperature-based version of the EM scheme (using the Hemlholtz's free energy as the thermodynamical potential and the temperature as the thermodynamical state variable). Appendix A includes an entropy-based analogue EM scheme (using the internal energy as the thermodynamical potential and the entropy as the thermodynamical state variable).

\section{Acknowledgements}

The first author acknowledges the support provided by Ministerio de Ciencia, Innovación y Universidades, for the award of a Juan de la Cierva Formación Fellowship. The first and third author acknowledge the support of AEI/FEDER and UE under the contracts DPI2016-77538-R and by Fundacion Séneca (Agencia de Ciencia y Tecnología de la Región de Murcia (Spain)) under the contract 20911/PI/18. The second author acknowledges the financial support received through the European Training Network Protection (Project ID: 764636). 


\section{Appendix A. Entropy-based EM scheme}

Although not pursued in this work, it is possible to define an entropy-based EM time integrator, counterpart of that in (48). For that we introduce first the entropy-based counterpart of the weak forms in (A.1), i.e.

$$
\begin{aligned}
& \mathcal{W}_{\boldsymbol{v}}=\int_{\mathcal{B}_{0}}(\boldsymbol{v}-\dot{\boldsymbol{\phi}}) \cdot \rho_{0} \boldsymbol{w}_{\boldsymbol{v}} d V=0 ; \\
& \mathcal{W}_{\boldsymbol{\phi}}=\int_{\mathcal{B}_{0}} \rho_{0} \dot{\boldsymbol{v}} \cdot \boldsymbol{w}_{\boldsymbol{\phi}} d V+\int_{\mathcal{B}_{0}} \boldsymbol{S}: \frac{1}{2} D \boldsymbol{C}\left[\boldsymbol{w}_{\phi}\right] d V-\int_{\mathcal{B}_{0}} \boldsymbol{f}_{0} \cdot \boldsymbol{w}_{\phi} d V-\int_{\partial_{\boldsymbol{t}} \mathcal{B}_{0}} \boldsymbol{t}_{0} \cdot \boldsymbol{w}_{\phi} d A=0 ; \\
& \mathcal{W}_{\eta}=\int_{\mathcal{B}_{0}} \theta \dot{\eta} w_{\eta} d V-\int_{\mathcal{B}_{0}} \boldsymbol{Q} \cdot \nabla_{0} w_{\eta} d V-\int_{\mathcal{B}_{0}} R_{\theta} w_{\eta} d V-\int_{\partial_{Q} \mathcal{B}_{0}} Q_{\theta} w_{\eta} d A=0,
\end{aligned}
$$

where $\{\boldsymbol{v}, \boldsymbol{\phi}, \eta\} \in \mathbb{V}^{\phi} \times \mathbb{V}^{\phi} \times \mathbb{V}^{\theta}$ and $\left.\left\{\boldsymbol{w}_{\boldsymbol{v}}, \boldsymbol{w}_{\boldsymbol{\phi}}, w_{\eta}\right\} \in \mathbb{V}_{0}^{\phi} \times \mathbb{V}_{0}^{\phi} \times \mathbb{V}_{0}^{\theta}\right\}$ in (39). Notice that in order to obtain $(\mathrm{A} .1)_{c}$, the classical local form in (5) needs to be used, rather than its equivalent couterpart in (6). In order to design the entropy-based EM momentum from (A.1), we strictly follow the steps enumerated in Remark 1, yielding

$$
\begin{aligned}
\left(\mathcal{W}_{\boldsymbol{v}}\right)_{\text {algo }} & =\int_{\mathcal{B}_{0}}\left(\boldsymbol{v}_{n+1 / 2}-\frac{\Delta \boldsymbol{\phi}}{\Delta t}\right) \cdot \rho_{0} \boldsymbol{w}_{\boldsymbol{v}} d V=0 ; \\
\left(\mathcal{W}_{\boldsymbol{\phi}}\right)_{\text {algo }} & =\int_{\mathcal{B}_{0}} \rho_{0} \frac{\Delta \boldsymbol{v}}{\Delta t} \cdot \boldsymbol{w}_{\boldsymbol{\phi}} d V+\int_{\mathcal{B}_{0}} \boldsymbol{S}_{\text {algo }}: \frac{1}{2}\left(D \boldsymbol{C}\left[\boldsymbol{w}_{\phi}\right]\right)_{\text {algo }} d V-\int_{\mathcal{B}_{0}} \boldsymbol{f}_{0_{n+1 / 2}} \cdot \boldsymbol{w}_{\boldsymbol{\phi}} d V \\
& -\int_{\partial_{t} \mathcal{B}_{0}} \boldsymbol{t}_{0_{n+1 / 2}} \cdot \boldsymbol{w}_{\boldsymbol{\phi}} d A=0 \\
\left(\mathcal{W}_{\eta}\right)_{\text {algo }} & =\int_{\mathcal{B}_{0}} \theta_{\text {algo }} \frac{\Delta \eta}{\Delta t} w_{\eta} d V-\int_{\mathcal{B}_{0}} \boldsymbol{Q}_{n+1 / 2} \cdot \nabla_{0} w_{\eta} d V-\int_{\mathcal{B}_{0}} R_{\theta_{n+1 / 2}} w_{\eta} d V-\int_{\partial_{Q} \mathcal{B}_{0}} Q_{\theta_{n+1 / 2}} w_{\eta} d A=0,
\end{aligned}
$$

where the algorithmic expressions $\left\{\boldsymbol{S}_{\text {algo }}, \theta_{\text {algo }}\right\}$ are defined as

$$
\boldsymbol{S}_{\text {algo }}=2 D_{\boldsymbol{C}} \widetilde{U}+2 D_{\boldsymbol{G}} \widetilde{U} \times \boldsymbol{C}_{\text {algo }}+2 D_{C} \widetilde{U} \boldsymbol{G}_{\text {algo }} ; \quad \theta_{\text {algo }}=D_{\eta} \widetilde{U},
$$

and with $\boldsymbol{C}_{\text {algo }}$ and $\boldsymbol{G}_{\text {algo }}$ in (50). Following a similar procedure to that in Sections 5.2.1 and 5.2.2, it is possible to prove that the EM time integrator in (A.2) preserves both linear and angular momentum for vanishing external forces. With regards to its energy conservation properties, we replace in (A.2) $\left\{\boldsymbol{w}_{\boldsymbol{v}}, \boldsymbol{w}_{\phi}\right\}$ with $\{\Delta \boldsymbol{v} / \Delta t, \Delta \boldsymbol{\phi} / \Delta t\} \in \mathbb{V}_{0}^{\phi} \times \mathbb{V}_{0}^{\phi}$ and $w_{\eta}=1$, yielding

$$
\frac{\Delta K}{\Delta t}+\int_{\mathcal{B}_{0}} \frac{1}{\Delta t}\left(D_{\boldsymbol{C}} \widetilde{U}: \Delta \boldsymbol{C}+D_{\boldsymbol{G}} \widetilde{U}: \Delta \boldsymbol{G}+D_{C} \widetilde{U} \Delta C+D_{\eta} \widetilde{U} \Delta \eta\right) d V-\frac{\Delta \prod_{\text {ext }}(\phi)}{\Delta t}-\mathcal{Q}_{\text {ext }}=0 .
$$

Therefore, from (A.4), the directionality property for the entropy-based formulation must be ${ }^{8}$

$$
D_{\boldsymbol{C}} \widetilde{U}: \Delta \boldsymbol{C}+D_{\boldsymbol{G}} \widetilde{U}: \Delta \boldsymbol{G}+D_{C} \widetilde{U} \Delta C+D_{\eta} \widetilde{U} \Delta \eta=\Delta \widetilde{U}
$$

A definition of the discrete derivatives $\left\{D_{C} \widetilde{U}, D_{G} \widetilde{U}, D_{C} \widetilde{U}, D_{\theta} \widetilde{U}\right\}$ based on the derivation presented in [17] for energies depending upon several arguments ensures the satisfaction of (A.5) (see also Appendix $\mathrm{B}$, where $\widetilde{W}$ and $\theta$ need to be simply replaced with $\widetilde{U}$ and $\eta$, respectively). Based on this definition of the discrete derivatives equation (A.4) can be finally written as

$$
\frac{\Delta K}{\Delta t}+\int_{\mathcal{B}_{0}} \frac{\Delta \widetilde{U}}{\Delta t} d V-\frac{\Delta \Pi_{\mathrm{ext}}(\phi)}{\Delta t}-\mathcal{Q}_{\mathrm{ext}}=0
$$

\footnotetext{
${ }^{8}$ see its temperature-based counterpart in $(52)$
} 
which is completely equivalent to the result obtained for the temperature-based counterpart in (68). Equation (A.6) ensures the consistency of the entropy-based EM time integrator (A.2). It is convenient to recall that the main drawback of entropy-based formulations is that in general, it might not be possible to find en explicit representation of the internal energy functional (and of its derivatives). However, for the Helmholtz functional presented in Section 3.1.1 we have been able to obtain a simple explicit expression for its associated internal energy (see (31) and (32)). 


\section{Appendix B. Discrete derivatives of the internal energy}

Appendix B.1. Definition of the discrete derivatives

Let us introduce the following notation, $\left\{\mathcal{V}_{1}, \mathcal{V}_{2}, \mathcal{V}_{3}, \mathcal{V}_{4}\right\}=\{\boldsymbol{C}, \boldsymbol{G}, C, \theta\}$. This will facilitate the definition of the discrete derivatives $D_{\widetilde{\mathcal{V}}_{1}} \widetilde{W}=D_{C} \widetilde{W}, D_{\widetilde{\mathcal{V}}_{2}} \widetilde{W}=D_{G} \widetilde{W}$ and $D_{\widetilde{\mathcal{V}}_{3}} \widetilde{W}=D_{C} \widetilde{W}$ in (49) and $D_{\widetilde{\mathcal{V}}_{4}} \widetilde{W}=D_{\theta} \widetilde{W}$ in $(48)_{d}$.

$$
\begin{array}{rlrl}
D_{\widetilde{\mathcal{V}}_{i}} \widetilde{W} & =\frac{1}{2}\left(D_{\widetilde{\mathcal{V}}_{i_{n+1, n}}} \widetilde{W}+D_{\widetilde{\mathcal{V}}_{i_{n, n+1}}} \widetilde{W}\right) ; & & i \in Y=\{1,2,3,4\} ; \\
D_{\widetilde{\mathcal{V}}_{i_{n+1}, n}} \widetilde{W}=\left.D_{\widetilde{\mathcal{V}}_{i}} \widetilde{W}\left(\widetilde{\mathcal{V}}_{i_{n+1}}, \widetilde{\mathcal{V}}_{i_{n}}\right)\right|_{\widetilde{\mathcal{V}}_{j_{n+1}}, \widetilde{\mathcal{V}}_{k_{n}}} ; & & \forall j \in Y: j<i ; \forall k \in Y: k>i ; \\
D_{\widetilde{\mathcal{V}}_{i_{n, n+1}} \widetilde{W}}=\left.D_{\widetilde{\mathcal{V}}_{i}} \widetilde{W}\left(\widetilde{\mathcal{V}}_{i_{n}}, \widetilde{\mathcal{V}}_{i_{n+1}}\right)\right|_{\widetilde{\mathcal{V}}_{j_{n}}, \widetilde{\mathcal{V}}_{k_{n+1}}} ; & & \forall j \in Y: j<i ; \forall k \in Y: k>i,
\end{array}
$$

where the discrete operators $\left.D_{\widetilde{\mathcal{V}}_{i}} \widetilde{W}\right|_{\widetilde{\mathcal{V}}_{j_{n+1}}, \widetilde{\mathcal{V}}_{k_{n}}}$ and $\left.D_{\widetilde{\mathcal{V}}_{i}} \widetilde{W}\right|_{\widetilde{\mathcal{V}}_{j_{n}}, \widetilde{\mathcal{V}}_{k_{n+1}}}$ are defined as

$$
\begin{aligned}
& \left.D_{\widetilde{\mathcal{V}}_{i}} \widetilde{W}\right|_{\widetilde{\mathcal{V}}_{j_{n+1}}, \widetilde{\mathcal{V}}_{k_{n}}}=\left.\partial_{\widetilde{\mathcal{V}}_{i}} \widetilde{W}\left(\widetilde{\mathcal{V}}_{n+1 / 2}\right)\right|_{\widetilde{\mathcal{V}}_{j_{n+1}}, \widetilde{\mathcal{V}}_{k_{n}}} \\
& +\frac{\left.\widetilde{W}\left(\widetilde{\mathcal{V}}_{n+1}\right)\right|_{\widetilde{\mathcal{V}}_{j_{n+1}}, \widetilde{\mathcal{V}}_{k_{n}}}-\left.\widetilde{W}\left(\widetilde{\mathcal{V}}_{n}\right)\right|_{\widetilde{\mathcal{V}}_{j_{n+1}}, \widetilde{\mathcal{V}}_{k_{n}}}-\left.\partial_{\widetilde{\mathcal{V}}_{i}} \widetilde{W}\left(\widetilde{\mathcal{V}}_{n+1 / 2}\right)\right|_{\widetilde{\mathcal{V}}_{j_{n+1}}, \widetilde{\mathcal{V}}_{k_{n}}}: \Delta \widetilde{\mathcal{V}}_{i}}{\left\|\Delta \widetilde{\mathcal{V}}_{i}\right\|^{2}} \Delta \widetilde{\mathcal{V}}_{i} ; \\
& \left.D_{\widetilde{\mathcal{V}}_{i}} \widetilde{W}\right|_{\widetilde{\mathcal{V}}_{j_{n}}, \widetilde{\mathcal{V}}_{k_{n+1}}}=\left.\partial_{\widetilde{\mathcal{V}}_{i}} \widetilde{W}\left(\widetilde{\mathcal{V}}_{n+1 / 2}\right)\right|_{\widetilde{\mathcal{V}}_{j_{n}}, \widetilde{\mathcal{V}}_{k_{n+1}}} \\
& +\frac{\left.\widetilde{W}\left(\widetilde{\mathcal{V}}_{n+1}\right)\right|_{\widetilde{\mathcal{V}}_{j_{n}}, \widetilde{\mathcal{V}}_{k_{n+1}}}-\left.\widetilde{W}\left(\widetilde{\mathcal{V}}_{n}\right)\right|_{\widetilde{\mathcal{V}}_{j_{n}}, \widetilde{\mathcal{V}}_{k_{n+1}}}-\left.\partial_{\widetilde{\mathcal{V}}_{i}} \widetilde{W}\left(\widetilde{\mathcal{V}}_{n+1 / 2}\right)\right|_{\widetilde{\mathcal{V}}_{j_{n}}, \widetilde{\mathcal{V}}_{k_{n+1}}}: \Delta \widetilde{\mathcal{V}}_{i}}{\left\|\Delta \widetilde{\mathcal{V}}_{i}\right\|^{2}} \Delta \widetilde{\mathcal{V}}_{i} .
\end{aligned}
$$

Let us introduce the following set $\widetilde{\mathcal{V}}_{\boldsymbol{C}}=\widetilde{\mathcal{V}} \backslash\{\boldsymbol{C}\}$, i.e. $\mathcal{V}_{\boldsymbol{C}}=\{\boldsymbol{G}, C, \theta\}$. From above equations (B.1) and (B.2), the directional derivative $D_{\boldsymbol{C}} \widetilde{W}$ can be computed as

$$
\begin{aligned}
D_{\boldsymbol{C}} \widetilde{W} & =\frac{1}{2}\left(\partial_{\boldsymbol{C}} \widetilde{W}\left(\boldsymbol{C}_{n+1 / 2}, \widetilde{\mathcal{V}}_{1_{n+1}}\right)+\partial_{\boldsymbol{C}} \widetilde{W}\left(\boldsymbol{C}_{n+1 / 2}, \widetilde{\mathcal{V}}_{\boldsymbol{C}_{n}}\right)\right) \\
& +\frac{1}{2} \frac{\widetilde{W}\left(\boldsymbol{C}_{n+1}, \widetilde{\mathcal{V}}_{1_{n+1}}\right)-\widetilde{W}\left(\boldsymbol{C}_{n}, \widetilde{\mathcal{V}}_{1_{n+1}}\right)}{\|\Delta \boldsymbol{C}\|^{2}} \Delta \boldsymbol{C}+\frac{1}{2} \frac{\widetilde{W}\left(\boldsymbol{C}_{n+1}, \widetilde{\mathcal{V}}_{\boldsymbol{C}_{n}}\right)-\widetilde{W}\left(\boldsymbol{C}_{n}, \widetilde{\mathcal{V}}_{\boldsymbol{C}_{n}}\right)}{\|\Delta \boldsymbol{C}\|^{2}} \Delta \boldsymbol{C} \\
& -\frac{1}{2} \frac{\partial_{\boldsymbol{C}} \widetilde{W}\left(\boldsymbol{C}_{n+1 / 2}, \widetilde{\mathcal{V}}_{1_{n+1}}\right): \Delta \boldsymbol{C}}{\|\Delta \boldsymbol{C}\|^{2}} \Delta \boldsymbol{C}-\frac{1}{2} \frac{\partial_{\boldsymbol{C}} \widetilde{W}\left(\boldsymbol{C}_{n+1 / 2}, \widetilde{\mathcal{V}}_{\boldsymbol{C}_{n}}\right): \Delta \boldsymbol{C}}{\|\Delta \boldsymbol{C}\|^{2}} \Delta \boldsymbol{C} .
\end{aligned}
$$

From the previous equation, the discrete derivatives with respect to $\boldsymbol{C}$ when $\widetilde{\mathcal{V}}_{\boldsymbol{C}_{n+1}}$ and $\widetilde{\mathcal{V}}_{\boldsymbol{C}_{n}}$ are kept fixed are defined as

$$
\begin{aligned}
D_{\boldsymbol{C}} \widetilde{W}\left(\bullet, \widetilde{\mathcal{V}}_{\boldsymbol{C}_{n+1}}\right):=\partial_{\boldsymbol{C}} \widetilde{W}\left(\boldsymbol{C}_{n+1 / 2}, \widetilde{\mathcal{V}}_{\boldsymbol{C}_{n+1}}\right) \\
+\frac{\widetilde{W}\left(\boldsymbol{C}_{n+1}, \widetilde{\mathcal{V}}_{\boldsymbol{C}_{n+1}}\right)-\widetilde{W}\left(\boldsymbol{C}_{n}, \widetilde{\mathcal{V}}_{\boldsymbol{C}_{n+1}}\right)-\partial_{\boldsymbol{C}} \widetilde{W}\left(\boldsymbol{C}_{n+1 / 2}, \widetilde{\mathcal{V}}_{\boldsymbol{C}_{n+1}}\right): \Delta \boldsymbol{C}}{\|\Delta \boldsymbol{C}\|^{2}} \Delta \boldsymbol{C} ; \\
D_{\boldsymbol{C}} \widetilde{W}\left(\bullet, \widetilde{\mathcal{V}}_{\boldsymbol{C}_{n}}\right):=\partial_{\boldsymbol{C}} \widetilde{W}\left(\boldsymbol{C}_{n+1 / 2}, \widetilde{\mathcal{V}}_{\boldsymbol{C}_{n}}\right) \\
+\frac{\widetilde{W}\left(\boldsymbol{C}_{n+1}, \widetilde{\mathcal{V}}_{\boldsymbol{C}_{n}}\right)-\widetilde{W}\left(\boldsymbol{C}_{n}, \widetilde{\mathcal{V}}_{\boldsymbol{C}_{n}}\right)-\partial_{\boldsymbol{C}} \widetilde{W}\left(\boldsymbol{C}_{n+1 / 2}, \widetilde{\mathcal{V}}_{\boldsymbol{C}_{n}}\right): \Delta \boldsymbol{C}}{\|\Delta \boldsymbol{C}\|^{2}} \Delta \boldsymbol{C} .
\end{aligned}
$$


Therefore, equation (B.3) can be conveniently written in a compact manner as

$$
D_{\boldsymbol{C}} \widetilde{W}=\frac{1}{2}\left(D_{\boldsymbol{C}} \widetilde{W}\left(\bullet, \widetilde{\mathcal{V}}_{\boldsymbol{C}_{n+1}}\right)+D_{\boldsymbol{C}} \widetilde{W}\left(\bullet, \widetilde{\mathcal{V}}_{\boldsymbol{C}_{n}}\right)\right)
$$

Similarly, defining the following sets $\widetilde{\mathcal{V}}_{\boldsymbol{G}}=\widetilde{\mathcal{V}} \backslash\{\boldsymbol{G}\}, \widetilde{\mathcal{V}}_{C}=\widetilde{\mathcal{V}} \backslash\{C\}$ and $\widetilde{\mathcal{V}}_{\theta}=\widetilde{\mathcal{V}} \backslash\{\theta\}$, it is possible to express the directional derivatives $D_{G} \widetilde{W}, D_{C} \widetilde{W}$ and $D_{\theta} \widetilde{W}$ as

$$
\begin{aligned}
D_{\boldsymbol{G}} \widetilde{W} & =\frac{1}{2}\left(D_{\boldsymbol{G}} \widetilde{W}\left(\bullet, \mathcal{V}_{\boldsymbol{G}_{n+1}}\right)+D_{\boldsymbol{G}} \widetilde{W}\left(\bullet, \mathcal{V}_{\boldsymbol{G}_{n}}\right)\right) ; \\
D_{C} \widetilde{W} & =\frac{1}{2}\left(D_{C} \widetilde{W}\left(\bullet, \mathcal{V}_{C_{n+1}}\right)+D_{C} \widetilde{W}\left(\bullet, \mathcal{V}_{C_{n}}\right)\right) ; \\
D_{\theta} \widetilde{W} & =\frac{1}{2}\left(D_{\theta} \widetilde{W}\left(\bullet, \mathcal{V}_{\theta_{n+1}}\right)+D_{\theta} \widetilde{W}\left(\bullet, \mathcal{V}_{\theta_{n}}\right)\right) .
\end{aligned}
$$

In the particular case of the last to directional derivatives (with respect to $C$ and $\theta$ ), the terms $D_{C} \widetilde{W}\left(\bullet, \mathcal{V}_{C_{n+1}}\right)$ (and $D_{C} \widetilde{W}\left(\bullet, \mathcal{V}_{C_{n}}\right)$ ) and $D_{\theta} \widetilde{W}\left(\bullet, \mathcal{V}_{\theta_{n+1}}\right)$ (and similarly $D_{\theta} \widetilde{W}\left(\bullet, \mathcal{V}_{\theta_{n}}\right)$ ) are extremely simplified since $C$ and $\theta$ are scalar fields, i.e.

$$
\begin{aligned}
D_{C} \widetilde{W}\left(\bullet, \mathcal{V}_{C_{n+1}}\right) & =\frac{\widetilde{W}\left(C_{n+1}, \mathcal{V}_{C_{n+1}}\right)-\widetilde{W}\left(C_{n}, \mathcal{V}_{C_{n+1}}\right)}{\Delta C} ; \\
D_{\theta} \widetilde{W}\left(\bullet, \mathcal{V}_{\theta_{n+1}}\right) & =\frac{\widetilde{W}\left(\theta_{n+1}, \mathcal{V}_{\theta_{n+1}}\right)-\widetilde{W}\left(\theta_{n}, \mathcal{V}_{\theta_{n+1}}\right)}{\Delta \theta} ;
\end{aligned}
$$

In particular, for the Mooney-Rivlin model in equation (19) and (26), the tensor discrete derivatives $\left\{D_{\boldsymbol{C}} \widetilde{W}, D_{\boldsymbol{G}} \widetilde{W}\right\}$ adopt the following extremely simple expressions

$$
D_{\boldsymbol{C}} \widetilde{W}=\frac{\mu_{1}}{2} \boldsymbol{I} ; \quad D_{\boldsymbol{G}} \widetilde{W}=\frac{\mu_{2}}{2} \boldsymbol{I} .
$$

Appendix B.2. Proof of directionality property

The objective of this section is to prove that the definition of the discrete derivatives of the internal energy $\widetilde{W}(\boldsymbol{C}, \boldsymbol{G}, C, \theta)$ in (B.1) and (B.2) satisfy the directionality property in equation (52). For that, let us denote the expression on the left-hand side of the directionality property in (52) as $\mathcal{T}$, namely

$$
\mathcal{T}=D_{\boldsymbol{C}} W: \Delta \boldsymbol{C}+D_{\boldsymbol{G}} W: \Delta \boldsymbol{G}+D_{C} W \Delta C+D_{\theta} W \cdot \Delta \theta
$$

Substitution of the expressions for $D_{C} \widetilde{W}$ (B.3), $D_{G} \widetilde{W}$ (B.6), $D_{C} \widetilde{W}$ (B.6) and $D_{\theta} \widetilde{W}$ (B.6) into (B.9) leads to

$$
\begin{aligned}
\mathcal{T} & =\frac{1}{2} \widetilde{W}\left(\boldsymbol{C}_{n+1}, \boldsymbol{G}_{n+1}, C_{n+1}, \theta_{n+1}\right)-\frac{1}{2} \widetilde{W}\left(\boldsymbol{C}_{n}, \boldsymbol{G}_{n+1}, C_{n+1}, \theta_{n+1}\right) \\
& +\frac{1}{2} \widetilde{W}\left(\boldsymbol{C}_{n+1}, \boldsymbol{G}_{n}, C_{n}, \theta_{n}\right)-\frac{1}{2} \widetilde{W}\left(\boldsymbol{C}_{n}, \boldsymbol{G}_{n}, C_{n}, \theta_{n}\right) \\
& +\frac{1}{2} \widetilde{W}\left(\boldsymbol{C}_{n}, \boldsymbol{G}_{n+1}, C_{n+1}, \theta_{n+1}\right)-\frac{1}{2} \widetilde{W}\left(\boldsymbol{C}_{n}, \boldsymbol{G}_{n}, C_{n+1}, \theta_{n+1}\right) \\
& +\frac{1}{2} \widetilde{W}\left(\boldsymbol{C}_{n+1}, \boldsymbol{G}_{n+1}, C_{n}, \theta_{n}\right)-\frac{1}{2} \widetilde{W}\left(\boldsymbol{C}_{n+1}, \boldsymbol{G}_{n}, C_{n}, \theta_{n}\right) \\
& +\frac{1}{2} \widetilde{W}\left(\boldsymbol{C}_{n}, \boldsymbol{G}_{n}, C_{n+1}, \theta_{n+1}\right)-\frac{1}{2} \widetilde{W}\left(\boldsymbol{C}_{n}, \boldsymbol{G}_{n}, C_{n}, \theta_{n+1}\right) \\
& +\frac{1}{2} \widetilde{W}\left(\boldsymbol{C}_{n+1}, \boldsymbol{G}_{n+1}, C_{n+1}, \theta_{n}\right)-\frac{1}{2} \widetilde{W}\left(\boldsymbol{C}_{n+1}, \boldsymbol{G}_{n+1}, C_{n}, \theta_{n}\right) \\
& +\frac{1}{2} \widetilde{W}\left(\boldsymbol{C}_{n}, \boldsymbol{G}_{n}, C_{n}, \theta_{n+1}\right)-\frac{1}{2} \widetilde{W}\left(\boldsymbol{C}_{n}, \boldsymbol{G}_{n}, C_{n}, \theta_{n}\right) \\
& +\frac{1}{2} \widetilde{W}\left(\boldsymbol{C}_{n+1}, \boldsymbol{G}_{n+1}, C_{n+1}, \theta_{n+1}\right)-\frac{1}{2} \widetilde{W}\left(\boldsymbol{C}_{n+1}, \boldsymbol{G}_{n+1}, C_{n+1}, \theta_{n}\right) \\
& =\Delta \widetilde{W}
\end{aligned}
$$


which proves that the definition of the discrete derivatives satisfy the directionality property.

\section{Appendix B.3. Definition of the discrete derivatives in the limit}

The objective of this section is to prove that the defition of the directional derivatives in equations (B.1) and (B.2) satisfies the second condition stated in Section 5.1.1, namely that they are well defined in the limit $\|\Delta \boldsymbol{C}\| \rightarrow 0,\|\Delta \boldsymbol{G}\| \rightarrow 0,\|\Delta C\| \rightarrow 0$ and $\|\Delta \theta\| \rightarrow 0$. In particular, it will be proved in this Section that based on the definition of the discrete derivatives, these can be equivalently written as

$$
D_{\widetilde{\mathcal{V}}_{i}} \widetilde{W}=\partial_{\widetilde{\mathcal{V}}_{i}} \widetilde{W}\left(\widetilde{\mathcal{V}}_{n+1 / 2}\right)+\sum_{i=1}^{4} O\left(\left\|\Delta \widetilde{\mathcal{V}}_{i}\right\|^{2}\right)+\sum_{j=1, j \neq i}^{4} \sum_{k=j+1, k \neq 1}^{4} O\left(\left\|\Delta \widetilde{\mathcal{V}}_{j}\right\|\left\|\Delta \widetilde{\mathcal{V}}_{k}\right\|\right)
$$

which would prove that they are well defined in the limit. For that, let us carry out a Taylor series expansion of the four different evaluations of the internal energy $\widetilde{W}$ in equation (B.3) around $\boldsymbol{C}_{n+1 / 2}$. This enables to express them as

$$
\begin{aligned}
\widetilde{W}\left(\boldsymbol{C}_{n+1}, \widetilde{\mathcal{V}}_{\boldsymbol{C}_{n+1}}\right) & =\widetilde{W}\left(\boldsymbol{C}_{n+1 / 2}, \widetilde{\mathcal{V}}_{\boldsymbol{C}_{n+1}}\right)+\partial_{\boldsymbol{C}} \widetilde{W}\left(\boldsymbol{C}_{n+1 / 2}, \widetilde{\mathcal{V}}_{\boldsymbol{C}_{n+1}}\right):\left(\frac{1}{2} \Delta \boldsymbol{C}\right) \\
& +\left(\frac{1}{2} \Delta \boldsymbol{C}\right): \partial_{\boldsymbol{C} \boldsymbol{C}}^{2} \widetilde{W}\left(\boldsymbol{C}_{n+1 / 2}, \widetilde{\mathcal{V}}_{\boldsymbol{C}_{n+1}}\right):\left(\frac{1}{2} \Delta \boldsymbol{C}\right)+O\left(\|\Delta \boldsymbol{C}\|^{3}\right) \\
\widetilde{W}\left(\boldsymbol{C}_{n}, \widetilde{\mathcal{V}}_{\boldsymbol{C}_{n+1}}\right) & =\widetilde{W}\left(\boldsymbol{C}_{n+1 / 2}, \widetilde{\mathcal{V}}_{\boldsymbol{C}_{n+1}}\right)-\partial_{\boldsymbol{C}} \widetilde{W}\left(\boldsymbol{C}_{n+1 / 2}, \widetilde{\mathcal{V}}_{\boldsymbol{C}_{n+1}}\right):\left(\frac{1}{2} \Delta \boldsymbol{C}\right) \\
& +\left(\frac{1}{2} \Delta \boldsymbol{C}\right): \partial_{\boldsymbol{C}}^{2} \widetilde{W}\left(\boldsymbol{C}_{n+1 / 2}, \widetilde{\mathcal{V}}_{\boldsymbol{C}_{n+1}}\right):\left(\frac{1}{2} \Delta \boldsymbol{C}\right)+O\left(\|\Delta \boldsymbol{C}\|^{3}\right) ; \\
\widetilde{W}\left(\boldsymbol{C}_{n+1}, \widetilde{\mathcal{V}}_{\boldsymbol{C}_{n}}\right) & =\widetilde{W}\left(\boldsymbol{C}_{n+1 / 2}, \widetilde{\mathcal{V}}_{\boldsymbol{C}_{n}}\right)+\partial_{\boldsymbol{C}} \widetilde{W}\left(\boldsymbol{C}_{n+1 / 2}, \widetilde{\mathcal{V}}_{\boldsymbol{C}_{n}}\right):\left(\frac{1}{2} \Delta \boldsymbol{C}\right) \\
& +\left(\frac{1}{2} \Delta \boldsymbol{C}\right): \partial_{\boldsymbol{C}}^{2} \widetilde{W}\left(\boldsymbol{C}_{n+1 / 2}, \widetilde{\mathcal{V}}_{\boldsymbol{C}_{n}}\right):\left(\frac{1}{2} \Delta \boldsymbol{C}\right)+O\left(\|\Delta \boldsymbol{C}\|^{3}\right) ; \\
\widetilde{W}\left(\boldsymbol{C}_{n}, \widetilde{\mathcal{V}}_{\boldsymbol{C}_{n}}\right) & =\widetilde{W}\left(\boldsymbol{C} n+1 / 2, \widetilde{\mathcal{V}}_{\boldsymbol{C}_{n}}\right)-\partial_{\boldsymbol{C}} \widetilde{W}\left(\boldsymbol{C}_{n+1 / 2}, \widetilde{\mathcal{V}}_{\boldsymbol{C}_{n}}\right):\left(\frac{1}{2} \Delta \boldsymbol{C}\right) \\
& +\left(\frac{1}{2} \Delta \boldsymbol{C}\right): \partial_{\boldsymbol{C}}^{2} \widetilde{W}\left(\boldsymbol{C}_{n+1 / 2}, \widetilde{\mathcal{V}}_{\boldsymbol{C}_{n}}\right):\left(\frac{1}{2} \Delta \boldsymbol{C}\right)+O\left(\|\Delta \boldsymbol{C}\|^{3}\right) .
\end{aligned}
$$

Introduction of above equation (B.12) into the last four terms on the right-hand side of equation (B.3) yields

$$
\begin{aligned}
& \frac{1}{2} \frac{\widetilde{W}\left(\boldsymbol{C}_{n+1}, \widetilde{\mathcal{V}}_{\boldsymbol{C}_{n+1}}\right)-\widetilde{W}\left(\boldsymbol{C}_{n}, \widetilde{\mathcal{V}}_{\boldsymbol{C}_{n+1}}\right)}{\|\Delta \boldsymbol{C}\|^{2}} \Delta \boldsymbol{C}+\frac{1}{2} \frac{\widetilde{W}\left(\boldsymbol{C}_{n+1}, \widetilde{\mathcal{V}}_{\boldsymbol{C}_{n}}\right)-\widetilde{W}\left(\boldsymbol{C}_{n}, \widetilde{\mathcal{V}}_{\boldsymbol{C}_{n}}\right)}{\|\Delta \boldsymbol{C}\|^{2}} \Delta \boldsymbol{C} \\
& -\frac{1}{2} \frac{\partial_{\boldsymbol{C}} \widetilde{W}\left(\boldsymbol{C}_{n+1 / 2}, \widetilde{\mathcal{V}}_{\boldsymbol{C}_{n+1}}\right): \Delta \boldsymbol{C}}{\|\Delta \boldsymbol{C}\|^{2}} \Delta \boldsymbol{C}-\frac{1}{2} \frac{\partial \boldsymbol{C}\left(\boldsymbol{C}_{n+1 / 2}, \widetilde{\mathcal{V}}_{\boldsymbol{C}_{n}}\right): \Delta \boldsymbol{C}}{\|\Delta \boldsymbol{C}\|^{2}} \Delta \boldsymbol{C}=O\left(\|\Delta \boldsymbol{C}\|^{2}\right) .
\end{aligned}
$$

Introduction of the result in (B.13) into the expression for the directional derivative $D_{\boldsymbol{C}} \widetilde{W}$ in (16) leads to

$$
D_{\boldsymbol{C}} \widetilde{W}=\frac{1}{2}\left(\partial_{\boldsymbol{C}} \widetilde{W}\left(\boldsymbol{C}_{n+1 / 2}, \widetilde{\mathcal{V}}_{\boldsymbol{C}_{n+1}}\right)+\partial_{\boldsymbol{C}} \widetilde{W}\left(\boldsymbol{C}_{n+1 / 2}, \widetilde{\mathcal{V}}_{\boldsymbol{C}_{n}}\right)\right)+O\left(\|\Delta \boldsymbol{C}\|^{2}\right)
$$


A Taylor series expansion on the two first terms on the right-hand side of above equation (B.14) enables these to be expressed as

$$
\begin{aligned}
\partial_{\boldsymbol{C}} \widetilde{W}\left(\boldsymbol{C}_{n+1 / 2}, \widetilde{\mathcal{V}}_{\boldsymbol{C}_{n+1}}\right) & =\partial_{\boldsymbol{C}} \widetilde{W}\left(\boldsymbol{C}_{n+1 / 2}, \widetilde{\mathcal{V}}_{\boldsymbol{C}_{n+1 / 2}}\right)+\partial_{\boldsymbol{C} \boldsymbol{G}}^{2} \widetilde{W}\left(\boldsymbol{C}_{n+1 / 2}, \widetilde{\mathcal{V}}_{\boldsymbol{C}_{n+1 / 2}}\right):\left(\frac{1}{2} \Delta \boldsymbol{G}\right) \\
& +\partial_{\boldsymbol{C} C}^{2} \widetilde{W}\left(\boldsymbol{C}_{n+1 / 2}, \widetilde{\mathcal{V}}_{\boldsymbol{C}_{n+1 / 2}}\right)\left(\frac{1}{2} \Delta C\right)+\partial_{\boldsymbol{C} \theta}^{2} \widetilde{W}\left(\boldsymbol{C}_{n+1 / 2}, \widetilde{\mathcal{V}}_{\boldsymbol{C}_{n+1 / 2}}\right):\left(\frac{1}{2} \Delta \theta\right) \\
& +O\left(\|\Delta \boldsymbol{G}\|^{2}\right)+O\left(\Delta C^{2}\right)+O\left(\|\Delta \theta\|^{2}\right) \\
& +O(\|\Delta \boldsymbol{G}\| \Delta C)+O(\|\Delta \boldsymbol{G}\|\|\Delta \theta\|)+O(\Delta C\|\Delta \theta\|) ; \\
\partial_{\boldsymbol{C}} \widetilde{W}\left(\boldsymbol{C}_{n+1 / 2}, \widetilde{\mathcal{V}}_{\boldsymbol{C}_{n}}\right) & =\partial_{\boldsymbol{C}} \widetilde{W}\left(\boldsymbol{C}_{n+1 / 2}, \widetilde{\mathcal{V}}_{\boldsymbol{C}_{n+1 / 2}}\right)-\partial_{\boldsymbol{C} \boldsymbol{G}}^{2} \widetilde{W}\left(\boldsymbol{C}_{n+1 / 2}, \widetilde{\mathcal{V}}_{\boldsymbol{C}_{n+1 / 2}}\right):\left(\frac{1}{2} \Delta \boldsymbol{G}\right) \\
& -\partial_{\boldsymbol{C} C}^{2} \widetilde{W}\left(\boldsymbol{C}_{n+1 / 2}, \widetilde{\mathcal{V}}_{\boldsymbol{C}_{n+1 / 2}}\right)\left(\frac{1}{2} \Delta C\right)-\partial_{\boldsymbol{C} \theta}^{2} \widetilde{W}\left(\boldsymbol{C}_{n+1 / 2}, \widetilde{\mathcal{V}}_{\boldsymbol{C}_{n+1 / 2}}\right):\left(\frac{1}{2} \Delta \theta\right) \\
& +O\left(\|\Delta \boldsymbol{G}\|^{2}\right)+O\left(\Delta C^{2}\right)+O\left(\|\Delta \theta\|^{2}\right) \\
& +O(\|\Delta \boldsymbol{G}\| \Delta C)+O(\|\Delta \boldsymbol{G}\|\|\Delta \theta\|)+O(\Delta C\|\Delta \theta\|) .
\end{aligned}
$$

Introduction of (B.15) into (B.14) leads to the final expression for $D_{\boldsymbol{C}} \widetilde{W}$ (B.3) as

$$
\begin{aligned}
D_{\boldsymbol{C}} \widetilde{W} & =\partial_{\boldsymbol{C}} \widetilde{W}\left(\boldsymbol{C}_{n+1 / 2}, \widetilde{\mathcal{V}}_{\boldsymbol{C}_{n+1 / 2}}\right) \\
& +O\left(\|\Delta \boldsymbol{C}\|^{2}\right)+O\left(\|\Delta \boldsymbol{G}\|^{2}\right)+O\left(\Delta C^{2}\right)+O\left(\|\Delta \theta\|^{2}\right) \\
& +O(\|\Delta \boldsymbol{G}\| \Delta C)+O(\|\Delta \boldsymbol{G}\|\|\Delta \theta\|)+O(\Delta C\|\Delta \theta\|)
\end{aligned}
$$

which proves condition (B.11). Proceeding similarly, it would be possible to generalise above result (B.16) to the discrete derivatives $D_{G} \widetilde{W}, D_{C} \widetilde{W}$ and $D_{\theta} \widetilde{W}$ (all of them in (B.6)), namely

$$
\begin{aligned}
D_{\boldsymbol{G}} \widetilde{W} & =\partial_{\boldsymbol{G}} \widetilde{W}\left(\boldsymbol{C}_{n+1 / 2}, \widetilde{\mathcal{V}}_{\boldsymbol{C}_{n+1 / 2}}\right) \\
& +O\left(\|\Delta \boldsymbol{C}\|^{2}\right)+O\left(\|\Delta \boldsymbol{G}\|^{2}\right)+O\left(\Delta C^{2}\right)+O\left(\|\Delta \theta\|^{2}\right) \\
& +O(\|\Delta \boldsymbol{C}\| \Delta C)+O(\|\Delta \boldsymbol{C}\|\|\Delta \theta\|)+O(\Delta C\|\Delta \theta\|) ; \\
D_{C} \widetilde{W} & =\partial_{C} \widetilde{W}\left(\boldsymbol{C}_{n+1 / 2}, \widetilde{\mathcal{V}}_{\boldsymbol{C}_{n+1 / 2}}\right) \\
& +O\left(\|\Delta \boldsymbol{C}\|^{2}\right)+O\left(\|\Delta \boldsymbol{G}\|^{2}\right)+O\left(\Delta C^{2}\right)+O\left(\|\Delta \theta\|^{2}\right) \\
& +O(\|\Delta \boldsymbol{C}\|\|\Delta \boldsymbol{G}\|)+O(\|\Delta \boldsymbol{C}\|\|\Delta \theta\|)+O(\|\Delta \boldsymbol{G}\|\|\Delta \theta\|) ; \\
D_{\theta} \widetilde{W} & =\partial_{\theta} \widetilde{W}\left(\boldsymbol{C}_{n+1 / 2}, \widetilde{\mathcal{V}}_{\boldsymbol{C}_{n+1 / 2}}\right) \\
& +O\left(\|\Delta \boldsymbol{C}\|^{2}\right)+O\left(\|\Delta \boldsymbol{G}\|^{2}\right)+O\left(\Delta C^{2}\right)+O\left(\|\Delta \theta\|^{2}\right) \\
& +O(\|\Delta \boldsymbol{C}\|\|\Delta \boldsymbol{G}\|)+O(\|\Delta \boldsymbol{C}\| \Delta C)+O(\|\Delta \boldsymbol{G}\| \Delta C) .
\end{aligned}
$$




\section{Appendix C. EM scheme in Reference [17]}

\section{Appendix C.1. EM scheme}

It is instructive to highlight the differences of the EM scheme in (48) and that previously developed in Reference [17]. The latter comprises the following algorithmic weak forms

$$
\begin{aligned}
\left(\mathcal{W}_{\boldsymbol{v}}\right)_{\text {algo }} & =\int_{\mathcal{B}_{0}}\left(\boldsymbol{v}_{n+1 / 2}-\frac{\Delta \boldsymbol{\phi}}{\Delta t}\right) \cdot \rho_{0} \boldsymbol{w}_{\boldsymbol{v}} d V=0 ; \\
\left(\mathcal{W}_{\boldsymbol{\phi}}\right)_{\text {algo }} & =\int_{\mathcal{B}_{0}} \rho_{0} \frac{\Delta \boldsymbol{v}}{\Delta t} \cdot \boldsymbol{w}_{\boldsymbol{\phi}} d V+\int_{\mathcal{B}_{0}} \boldsymbol{S}_{\text {algo }}: \frac{1}{2}\left(D \boldsymbol{C}\left[\boldsymbol{w}_{\phi}\right]\right)_{\text {algo }} d V-\int_{\mathcal{B}_{0}} \boldsymbol{f}_{0_{n+1 / 2}} \cdot \boldsymbol{w}_{\phi} d V \\
& -\int_{\partial_{t} \mathcal{B}_{0}} \boldsymbol{t}_{0_{n+1 / 2}} \cdot \boldsymbol{w}_{\boldsymbol{\phi}} d A=0 \\
\left(\mathcal{W}_{\theta}\right)_{\text {algo }} & =\int_{\mathcal{B}_{0}} \frac{\Delta \theta}{\Delta t} w_{\theta} d V+\int_{\mathcal{B}_{0}}\left(\frac{\Delta \boldsymbol{C}}{\Delta t}: \boldsymbol{G}_{n+1 / 2}\right)\left(D_{\theta} \eta\right)^{-1}\left(\partial_{C} \eta_{n+1 / 2}\right) w_{\theta} d V \\
& -\int_{\mathcal{B}_{0}} \boldsymbol{Q}_{n+1 / 2} \cdot \nabla_{0}\left(\left(D_{\theta} \hat{U}\right)^{-1} w_{\theta}\right) d V-\int_{\mathcal{B}_{0}}\left(D_{\theta} \hat{U}\right)^{-1} R_{\theta n+1 / 2} w_{\theta} d V \\
& -\int_{\partial_{Q} \mathcal{B}_{0}}\left(D_{\theta} \hat{U}\right)^{-1} Q_{\theta_{n+1 / 2}} w_{\theta} d A=0 .
\end{aligned}
$$

In above equation (C.1), $\boldsymbol{S}_{\text {algo }}$ is defined as

$$
\boldsymbol{S}_{\text {algo }}=2\left(D_{\boldsymbol{C}} \hat{U}+D_{\boldsymbol{G}} \hat{U} \times \boldsymbol{C}_{\text {algo }}+D_{C} \hat{U} \boldsymbol{G}_{\text {algo }}-\theta_{\text {algo }} \partial_{C} \eta_{n+1 / 2} \boldsymbol{G}_{n+1 / 2}\right) ; \quad \theta_{\text {algo }}=D_{\theta} \hat{U}\left(D_{\theta} \eta\right)^{-1},
$$

with $\boldsymbol{C}_{\text {algo }}$ and $\boldsymbol{G}_{\text {algo }}$ in (50) and where the internal energy energy functional $\hat{U}$ is defined as in equation (28), i.e.

$$
\hat{U}(\boldsymbol{C}, \boldsymbol{G}, C, \theta)=\widetilde{U}(\boldsymbol{C}, \boldsymbol{G}, C, \eta(C, \theta))=\theta \eta(C, \theta)+\widetilde{W}(\boldsymbol{C}, \boldsymbol{G}, C, \theta),
$$

with the particularity that the entropy is re-expressed as a function $\theta$ (and of $C$ ). Apart from the fact that this formulation relies on the internal energy functional $\hat{U}(\boldsymbol{C}, \boldsymbol{G}, C, \theta)$ (as opposed to the Helmholtz free energy functional $\widetilde{W}(\boldsymbol{C}, \boldsymbol{G}, C, \theta)$ for the EM scheme in (48)), the main differences between both approaches are:

1. The EM scheme in (C.1) relies on the local form (5), or more specifically on

$$
\dot{\eta}+\frac{1}{\theta} \mathrm{DIV} \boldsymbol{Q}-\frac{1}{\theta} R_{\theta}=0
$$

wheareas the proposed scheme in (48) relies on the local form in (6).

2. The algorithmic stresses $\boldsymbol{S}_{\text {algo }}$ in (C.2) (for the EM scheme in C.1) and in (49) (for the proposed EM scheme in (48)) differ considerably. In particular, the expression in equation (C.2) needs to incorporate a fourth term not present in equation (49). Notice that in the more generic case where the entropy could possibly depend also on $\boldsymbol{C}$ and $\boldsymbol{G}$ (and not just on $C$, as it has been assumed in this paper), this would entail the addition of two extra terms in (C.2) related to both $\boldsymbol{C}$ and $\boldsymbol{G}$, bringing cumbersome difficulties in the formulation.

3. The second term on the right hand side of equation (C.1) (which entails more complexity for a consistent linearisation of the set of weak forms) is not present in the proposed EM scheme in (48).

4. The term $\nabla_{0}\left(\left(D_{\theta} \hat{U}\right)^{-1} w_{\theta}\right)$ on equation $(\text { C.1 })_{c}$ can potentially entail excessive complexity when carrying out a consistent linearisation of $(\mathrm{C} .1)_{c}$. For the specific model considered in equations (26), (21) and (22) this is not the case, as this term is constant.

5. The EM scheme in (C.1) requires the definition of the discrete derivatives of the internal energy functional $\hat{U}(\boldsymbol{C}, \boldsymbol{G}, C, \theta)$ and in addition, the discrete derivative of the entropy $\eta(C, \theta)$, namely $D_{\theta} \eta\left(\right.$ see $\left.(\text { C. } 1)_{c}\right)$. 


\section{Appendix D. Thermo-elastic constitutive model}

The objective of this appendix is to briefly recall the calorimetry considerations followed in order to derive the constitutive model presented in Section 3.1.1. For that, we start by reexpressing the internal energy $\widetilde{e}(\boldsymbol{C}, \eta)$ as a function of the absolute temperature as

$$
\hat{e}(\boldsymbol{C}, \theta)=\widetilde{e}(\boldsymbol{C}, \eta(\boldsymbol{C}, \theta)) .
$$

Calorimetry principles permit to experimentally measure the change of internal energy as a function of the temperature (for a constant deformation) yielding

$$
\partial_{\theta} \hat{e}=c_{v}
$$

with $c_{v}$ denoting the heat capacity of the material. Notice that above equation (D.2) can be equivalently written as

$$
\partial_{\theta} \hat{e}=\partial_{\eta} \widetilde{e} \partial_{\theta} \eta=\theta \partial_{\theta} \eta=c_{v} \Rightarrow \partial_{\theta} \eta=\frac{c_{v}}{\theta} .
$$

Integration of (D.3) results in

$$
\int_{\eta\left(\boldsymbol{C}, \theta_{R}\right)}^{\eta(\boldsymbol{C}, \theta)} d \eta=\int_{\theta_{R}}^{\theta} \frac{c_{v}}{\theta} d \theta \Rightarrow \eta(\boldsymbol{C}, \theta)=\eta_{R}(\boldsymbol{C})+c_{v} \ln \frac{\theta}{\theta_{R}},
$$

with $\eta_{R}(\boldsymbol{C}):=\eta\left(\boldsymbol{C}, \theta_{R}\right)$. Since $\eta=-\partial_{\theta} \widetilde{\Psi}$ we can further integrate (D.4) as

$$
\int_{\widetilde{\Psi}\left(\boldsymbol{C}, \theta_{R}\right)}^{\widetilde{\Psi}(\boldsymbol{C}, \theta)} d \widetilde{\Psi}=-\int_{\theta_{R}}^{\theta} \eta(\boldsymbol{C}, \theta) d \theta
$$

yielding

$$
\begin{aligned}
\widetilde{\Psi}(\boldsymbol{C}, \theta) & =\widetilde{\Psi}\left(\boldsymbol{C}, \theta_{R}\right)-\int_{\theta_{R}}^{\theta}\left(\eta_{R}(\boldsymbol{C})+c_{v} \ln \frac{\theta}{\theta_{R}}\right) d \theta \\
& =\widetilde{\Psi}\left(\boldsymbol{C}, \theta_{R}\right)-\left(\theta-\theta_{R}\right) \eta_{R}(\boldsymbol{C})+c_{v}\left(\theta-\theta_{R}-\theta \ln \frac{\theta}{\theta_{R}}\right),
\end{aligned}
$$

which can be finally written as

$$
\widetilde{\Psi}(\boldsymbol{C}, \theta)=\widetilde{\Psi}_{m}(\boldsymbol{C})-\eta_{R}(\boldsymbol{C})\left(\theta-\theta_{R}\right)+\widetilde{\Psi}_{\theta}(\theta),
$$

with

$$
\widetilde{\Psi}_{m}(\boldsymbol{C})=\widetilde{\Psi}\left(\boldsymbol{C}, \theta_{R}\right) ; \quad \widetilde{\Psi}_{\theta}(\theta)=c_{v}\left(\theta-\theta_{R}-\theta \ln \frac{\theta}{\theta_{R}}\right)
$$




\section{References}

[1] J. M. Ball. Convexity conditions and existence theorems in nonlinear elasticity. Archive for Rational Mechanics and Analysis, 63(4):337-403, 1976.

[2] J. M. Ball. Energy-minimising configurations in nonlinear elasticity. Archive for Rational Mechanics and Analysis, 63(4):337-403, 1976.

[3] J. M. Ball. Geometry, Mechanics and Dynamics, chapter Some open problems in Elasticity, pages 3-59. Springer, 2002.

[4] J. M. Ball and F. Murat. $\mathrm{W}^{1, p}$-quasiconvexity and variational problems for multiple integrals. Journal of Functional Analysis, 58(3):225-253, 1984.

[5] K. J. Bathe. Finite Element Procedures. Prentice Hall, 1996.

[6] P. Betsch and A. Janz. An energy-momentum consistent method for transient simulations with mixed finite elements developed in the framework of geometrically exact shells. Int. J. Numer. Meth. Engng, 108(5):423-455, 2016.

[7] P. Betsch, A. Janz, and C. Hesch. A mixed variational framework for the design of energymomentum schemes inspired by the structure of polyconvex stored energy functions. Computer Methods in Applied Mechanics and Engineering, 335:660-696, 2018.

[8] J. Bonet, A. J. Gil, C. H. Lee, M. Aguirre, and R. Ortigosa. A first order hyperbolic framework for large strain computational solid dynamics - Part I: Total Lagrangian isothermal elasticity. Computer Methods in Applied Mechanics and Engineering, 283(0):689-732, 2015.

[9] J. Bonet, A. J. Gil, and R. Ortigosa. A computational framework for polyconvex large strain elasticity. Computer Methods in Applied Mechanics and Engineering, 283:1061-1094, 2015.

[10] J. Bonet, A. J. Gil, and R. Ortigosa. On a tensor cross product based formulation of large strain solid mechanics. International Journal of Solids and Structures, 84:49-63, 2016.

[11] J. Bonet, A. J. Gil, and R. D. Wood. Nonlinear Continuum Mechanics for Finite Element Analysis: Statics. Cambridge University Press, 2016.

[12] J. Bonet, C. H. Lee, A. J. Gil, and A. Ghavamian. A first order hyperbolic framework for large strain computational solid dynamics - Part III: Thermo-elasticity. Computer Methods in Applied Mechanics and Engineering. Under review.

[13] C. Christoforou, M. Galanopoulou, and A. E. Tzavaras. A symmetrizable extension of polyconvex thermoelasticity and applications to zero-viscosity limits and weak-strong uniqueness. Communications in Partial Differential Equations, 0(0):1-32, 2018.

[14] C. M. Dafermos. Hyperbolic conservation laws in continuum physics. Springer, 2010.

[15] R. de Boer. Vektor- und Tensorrechnung für Ingenieure. Springer-Verlag, 1982.

[16] E.A. de Souza Neto, D. Períc, and D.R.J. Owen. Computational Methods for Plasticity. Theory and Applications. 2008.

[17] M Franke, A. Janz, M. Schiebl, and P. Betsch. An energy momentum consistent integration scheme using a polyconvexity-based framework for nonlinear thermo-elastodynamics. International Journal for Numerical Methods in Engineering, 115:549-577, 2018. 
[18] O. Gonzalez. Exact energy and momentum conserving algorithms for general models in nonlinear elasticity. Comput. Methods Appl. Mech. Engrg., 190:1763-1783, 2000.

[19] O. Gonzalez and A. M. Stuart. A first course in Continuum Mechanics. Cambridge University Press, 2008.

[20] M. Groß and P. Betsch. Energy-momentum consistent finite element discretization of dynamic finite viscoelasticity. Int. J. Numer. Meth. Engng, 81(11):1341-1386, 2010.

[21] C. Hesch and P. Betsch. Energy-momentum consistent algorithms for dynamic thermomechanical problems - Application to mortar domain decomposition problems. International Journal for Numerical Methods in Engineering, 86(11):1277-1302, 2011.

[22] D. Kuhl and M. A. Crisfield. Constraint energy momentum algorighm and its application to non-linear structural dynamics of shells. Computer Methods in Applied Mechanics and Engineering, 136:293-315, 1996.

[23] D. Kuhl and M. A. Crisfield. Energy-conserving and decaying algorithms in non-linear structural dynamics. International Journal for Numerical Methods in Engineering, 45:565599, 1999.

[24] J. E. Marsden and T. J. R. Hughes. Mathematical foundations of elasticity. 1994.

[25] S. C. Martín and J. C. García Orden. On energy-entropy-momentum integration methods for discrete thermo-visco-elastodynamics. Computers \& Structures, 181:3-20, $2017 . \quad$ UK Association of Computational Mechanics.

[26] C. Miehe, D. Vallicotti, and D. Zäh. Computational structural and material stability analysis in finite electro-elasto-statics of electro-active materials. International Journal for Numerical Methods in Engineering, 102(10):1605-1637, 2015. nme.4855.

[27] R. Ortigosa, M. Franke, A. Janz, A.J. Gil, and P. Betsch. An energy-momentum time integration scheme based on a convex multi-variable framework for non-linear electroelastodynamics. Computer Methods in Applied Mechanics and Engineering, 339:1-35, 2018.

[28] I. Romero. An analysis of the stress formula for energy-momentum methods in nonlinear elastodynamics. Computational Mechanics, 50(5):603-610, Nov 2012.

[29] Ignacio Romero. Algorithms for coupled problems that preserve symmetries and the laws of thermodynamics: Part I: Monolithic integrators and their application to finite strain thermoelasticity. Computer Methods in Applied Mechanics and Engineering, 199(25):1841 1858, 2010.

[30] J. Schröder. Anisotropic polyconvex energies. In J. Schröder and P. Neff, editors, Poly-, quasiand rank-one convexity in Applied Mechanics, volume 516 of CISM Courses and Lectures, volume 516 of CISM Courses and Lectures, pages 53-105. Springer-Verlag, 2010.

[31] J. C. Simo and N. Tarnow. The discrete energy-momentum method. Conserving algorithms for nonlinear elastodynamics. Zeitschrift für angewandte Mathematik und Physik ZAMP, 43(5):757-792, Sep 1992. 